\title{
Matching and Morphing of Isometric Models
}

\author{
By \\ Stefanie Wuhrer \\ A thesis submitted to \\ the Faculty of Graduate Studies and Research \\ in partial fulfilment of \\ the requirements for the degree of \\ Doctor of Philosophy
}

Ottawa-Carleton Institute for Computer Science

School of Computer Science

Carleton University

Ottawa, Ontario

March 2009

(C) Copyright

2009, Stefanie Wuhrer 
Library and

Archives Canada

Published Heritage

Branch

395 Wellington Street

Ottawa ON K1A ON4

Canada
Bibliothèque et

Archives Canada

Direction du

Patrimoine de l'édition

395 , rue Wellington

Ottawa ON K1A ON4

Canada

Your file Votre référence

ISBN: 978-0-494-52074-1

Our file Notre référence

ISBN: 978-0-494-52074-1

NOTICE:

The author has granted a nonexclusive license allowing Library and Archives Canada to reproduce, publish, archive, preserve, conserve, communicate to the public by telecommunication or on the Internet, loan, distribute and sell theses worldwide, for commercial or noncommercial purposes, in microform, paper, electronic and/or any other formats.

The author retains copyright ownership and moral rights in this thesis. Neither the thesis nor substantial extracts from it may be printed or otherwise reproduced without the author's permission.
AVIS:

L'auteur a accordé une licence non exclusive permettant à la Bibliothèque et Archives Canada de reproduire, publier, archiver, sauvegarder, conserver, transmettre au public par télécommunication ou par l'Internet, prêter, distribuer et vendre des thèses partout dans le monde, à des fins commerciales ou autres, sur support microforme, papier, électronique et/ou autres formats.

L'auteur conserve la propriété du droit d'auteur et des droits moraux qui protège cette thèse. $\mathrm{Ni}$ la thèse ni des extraits substantiels de celle-ci ne doivent être imprimés ou autrement reproduits sans son autorisation.
In compliance with the Canadian

Privacy Act some supporting forms may have been removed from this thesis.

While these forms may be included in the document page count, their removal does not represent any loss of content from the thesis.
Conformément à la loi canadienne sur la protection de la vie privée, quelques formulaires secondaires ont été enlevés de cette thèse.

Bien que ces formulaires aient inclus dans la pagination, il n'y aura aucun contenu manquant.

\section{Canada}




\section{Abstract}

We present an approach to find dense point-to-point correspondences between two deformed surfaces corresponding to different postures of the same non-rigid object in a fully automatic way. The approach requires no prior knowledge about the shapes being registered or the initial alignment of the shapes. We consider surfaces that are represented by possibly incomplete triangular meshes. We model the deformations of an object as isometries. To solve the correspondence problem, our approach maps the intrinsic geometries of the surfaces into a low-dimensional Euclidean space via multidimensional scaling. This allows us to compute a number of candidate correspondences. The best candidate correspondence is found based on a deformation cost. 


\section{Acknowledgments}

First and foremost, I thank my supervisors Prosenjit Bose and Chang Shu for their continual advice and various support. Being experts in the fields of computational geometry and computer vision, my supervisors shared their knowledge and experience and always offered encouragement and support.

Furthermore, I thank Tetsuo Asano, Zouhour Ben Azouz, Alan Brunton, Paz Carmi, Anil Maheshwari, Joseph O'Rourke, and Michiel Smid, who shared their expertise and collaborated with me on topics included in this thesis. I also thank the members of my thesis committee for helpful discussions.

Last but not least, I thank the National Research Council of Canada, the Computational Geometry Group at Carleton University, the School of Computer Science at Carleton University, Graduate Studies at Carleton University, the High Performance Computing Virtual Laboratory, and the Ontario Student Assistantship Program for their generous financial support. 


\section{Contents}

1 Introduction $\quad 1$

2 Related Work 6

2.1 Correspondence Problem . . . . . . . . . . . . . . . . . . 6

2.2 Morphing . . . . . . . . . . . . . . . . . . . . 10

2.3 Multi-Dimensional Scaling . . . . . . . . . . . . . 13

2.3.1 Classical Multi-Dimensional Scaling . . . . . . . . . . . . 14

2.3.2 Least-Squares Multi-Dimensional Scaling . . . . . . . . . . . 15

2.3.3 Multi-Dimensional Scaling in Geometry Processing . . . . . . 17

2.4 The Fast Marching Method . . . . . . . . . . . . . . . . 18

2.5 Voronoi Sampling . . . . . . . . . . . . . . . . . . 19

3 Approximating Geodesics $\quad 21$

3.1 Introduction . . . . . . . . . . . . . . . . 22

3.2 Adding an Object to the Multi-Dimensional Scaling Embedding . . . 23

3.3 Estimating Geodesic Distances . . . . . . . . . . . . . . . 25

3.3 .1 Sampling Strategies . . . . . . . . . . . . . . . 27

3.3.2 Error Bounds of the Approximation . . . . . . . . . . 28

3.4 Description of the Algorithm . . . . . . . . . . . 28

3.5 Analysis of the Algorithm ................... 30

3.6 Experimental Results . . . . . . . . . . . . . . . . 31

3.7 Conclusion ......................... 41

$\mathrm{V}$ 
4 Graph Embedding in Linear Space $\quad 42$

4.1 Introduction . . . . . . . . . . . . . . . . . . . . . 43

4.2 Related Work . . . . . . . . . . . . . . . . . . . . 44

4.3 Clustering . . . . . . . . . . . . . . . . . . . . 48

4.3.1 Well-Separated Partitions . . . . . . . . . . . 49

4.3.2 Farthest and Most Compact $k$-Partition . . . . . . . . . 53

4.3.3 Size-Constrained Farthest Partition . . . . . . . . . . . . 54

4.4 Graph Embedding . . . . . . . . . . . . . . . 58

4.5 Experimental Results . . . . . . . . . . . . . . . . . 61

4.5.1 Embedding Registered High-Energy Gamma Particles . . . . . 62

4.5.2 Embedding Grey-Level Images . . . . . . . . . . . . . . . . . 63

4.5.3 Computing a Canonical Form . . . . . . . . . . . . 66 66

4.5 .4 Summary ... . . . . . . . . . . . . 69

4.6 Conclusions ......................... 70

5 Posture Invariant Correspondence $\quad \mathbf{7 2}$

5.1 Introduction . . . . . . . . . . . . . . . . . . . 72

5.2 Correspondence via Least-Squares Multi-

Dimensional Scaling . . . . . . . . . . . . . . . 74

5.3 Description of the Algorithm . . . . . . . . . . . . . 75

5.3 .1 Coarse Correspondence . . . . . . . . . . . 75

5.3 .2 Fine Correspondence . . . . . . . . . . . . . 78

5.4 Analysis of the Algorithm . . . . . . . . . . . . . . 80

5.5 Experimental Results . . . . . . . . . . . . . . . . . 81

5.6 Conclusion . . . . . . . . . . . . . . 87

6 Morphing of Triangular Meshes in Shape Space $\quad 91$

6.1 Introduction . . . . . . . . . . . . . . . . . . . . . . 92

6.2 Theory of Shape Space for Triangulated $3 D$ Polygons . . . . . . . . . 93

6.3 Theory of Shape Space for Skeleton Morphing . . . . . . . . . . . . . 98

6.4 Generalization to Triangular Meshes . . . . . . . . . . . . . . 100

vi 
6.5 Efficient Algorithm to Deform Triangular

Meshes . . . . . . . . . . . . . . . . . . . . . 104

6.6 Experimental Results . . . . . . . . . . . . . . . . . 105

6.6.1 Deforming Triangulated 3D Polygons . . . . . . . . . . . 105

6.6.2 Deforming General Triangular Meshes . . . . . . . . . . 106

6.7 Application to Segmentation Into Near-Rigid Components . . . . . . 109

6.8 Conclusion . . . . . . . . . . . . . . . . . . . . 113

7 Combining Correspondence and Shape Space $\mathbf{1 1 5}$

7.1 Introduction . . . . . . . . . . . . . . . . . 116

7.2 Overview of the Algorithm . . . . . . . . . . . . 116

7.3 Finding Candidate Correspondences Via Multi-Dimensional Scaling • 117

7.4 Choosing a Candidate Correspondence . . . . . . . . . . . . . . . . 119

7.4.1 Computing a Common Mesh $M \ldots \ldots$. . . . . . . . 119

7.4.2 Evaluating a Cost Function . . . . . . . . . . . . . 120

7.5 Experimental Results . . . . . . . . . . . . . . . . . . . 121

7.5.1 Correspondence Results . . . . . . . . . . . . . . 121

7.5.2 Application to Morphing . . . . . . . . . . . . . . . . . 125

7.5.3 Application to Finding Near-Rigid Components . . . . . . . . 127

7.6 Conclusion . . . . . . . . . . . . . . . 127

$\begin{array}{llr}8 & \text { Conclusion } & 129\end{array}$

$\begin{array}{ll}\text { Bibliography } & 131\end{array}$ 


\section{List of Tables}

3.1 Quality of approximation. The table shows the percentage of estimated errors with finite error bounds within relative error bounds of $0.25,0.5,0.75$, and 1 , respectively. A relative error bound of 0.5 implies that the true geodesic distance is at least half and at most 1.5 times the estimated geodesic distance. . . . . . . . . . . . . . .

4.1 Quality of embedding for the gamma telescope dataset. The table shows the maximum number $t$ of iterations required by the LSBFGS quasiNewton method [73], the embedding error $E_{L S}$ of the computed embedding, the storage use $S$ in $M B$, and the running time in seconds of all three algorithms that were implemented. . . . . . . . . . . . . . 63

4.2 Quality of embedding for the Yale Face Database. . . . . . . . . . . 66

4.3 Quality of embedding for the swiss roll. The table shows the maximum number $t$ of iterations required by the LSBFGS quasi-Newton method [73], the embedding error $E_{L S}$ of the computed embedding, the storage use $S$ in $M B$, and the running time in seconds of all three algorithms that were implemented. . . . . . . . . . . . . . . .

4.4 Quality of embedding for the Alien. The table shows the maximum number $t$ of iterations required by the LSBFGS quasi-Newton method [73], the embedding error $E_{L S}$ of the computed embedding, and the storage use $S$ of all three algorithms that were implemented. . . . . . . . . . .

viii 


\section{List of Figures}

3.1 Paths computed via the fast marching technique tracing boundaries of holes. . . . . . . . . . . . . . . . . . . . . . .

3.2 Chart describing the overview of the proposed algorithm. The left side of the chart describes the preprocessing performed once per surface. The right side of the chart describes the steps taken to compute the geodesic distance between two points on the surface after preprocessing. . . . .

3.3 (a), (c) Model of a bag obtained by image-based reconstruction. The points used to compute the canonical form are shown in red and the test vertices are shown in green. (b), (d) Canonical form of the bag obtained via least-squares MDS. (a), (b) Obtained by curvature-based sampling, (c), (d) Obtained by Voronoi sampling. . . . . . . . . . . . 33

3.4 Bag model with red dot as source point for SSSP computation. . . . . 34

3.5 (a) Color display of the geodesic distance estimates from one source point. Blue means small distance and red means large distance. (b) Color display of the error bounds of the distances shown in (a). Blue means small error bound and red means large error bound. (c) Detail

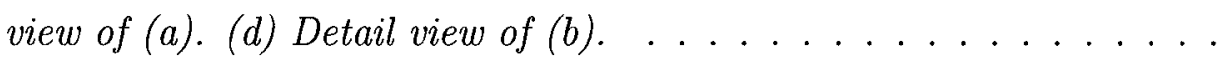

3.6 Model csr0001a. (a), (c) The points used to compute the canonical form are shown in red and the test vertices are shown in green. (b), (d) Canonical form of csr0001a obtained via least-squares MDS. (a), (b) Obtained by curvature-based sampling, (c), (d) Obtained by Voronoi sampling. ...................... 35

3.7 Model csr0001a. Detail views of holes on arm and upper body. . . . .

ix 
3.8 Model csr0106a. (a), (c) The points used to compute the canonical form are shown in red and the test vertices are shown in green. (b), (d) Canonical form of csr0106a obtained via least-squares MDS. (a), (b) Obtained by curvature-based sampling, (c), (d) Obtained by Voronoi

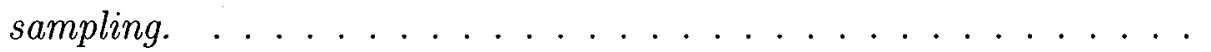

3.9 Model of a human model. Left: Complete model. Right: Modified model. The points used to compute the canonical form are shown in red and the test vertices are shown in green. . . . . . . . . . . . .

3.10 (a) Histogram shows the number of times that relative error bound in a certain bin occurs. The occurrence of $\hat{\delta}_{i, j}^{\text {upper }}$ is shown in white, the occurrence of $d_{i, j}$ is shown in black, and the occurrence of $e_{i, j}$ is shown in grey. (b) The columns show the percentage of distances where the relative error of $d_{i, j}$ is smaller than or equal to the relative error of $\hat{\delta}_{i, j}^{u p p e r}$. 40

3.11 Left: Complete model of a human head used to compute the geodesic distance between the two red points via the fast marching technique. Right: Incomplete model of the same head used to compute the geodesic estimate between the two red points using the approach presented in this chapter. . . . . . . . . . . . . . .

4.1 Path $p_{a i}$ comprised of short edges between $i$ and $a . \ldots \ldots \ldots$. 57

4.2 Example showing the result of running the clustering algorithm on a random set of 268 points in the plane. . . . . . . . . . . . .

4.3 Comparison of time and space used by the embedding algorithm (solid), the projection algorithm (dotted), and the SMACOF algorithm (dashed) for the gamma telescope dataset. The number of objects $n$ is shown along the $x$-axis and the space and time requirements are shown along

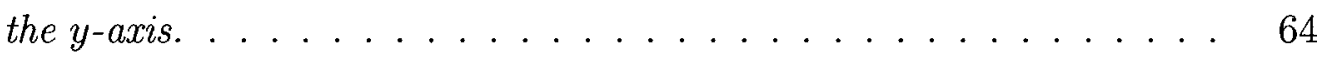

4.4 Facial expressions in the database. . . . . . . . . . . . 64 
4.5 The Figure shows the embedding results for the face database. Figure (a): embedding computed by the embedding algorithm. Figure (b): embedding computed by the projection algorithm. Figure (c): embedding computed by $S M A C O F \ldots \ldots \ldots \ldots \ldots \ldots \ldots$

4.6 Comparison of time and space used by the embedding algorithm (solid), the projection algorithm (dotted), and the SMACOF algorithm (dashed) for the swiss roll. The number of objects $n$ is shown along the $x$-axis and the space and time requirements are shown along the $y$-axis. . . .

4.7 Comparison of time and space used by the embedding algorithm (solid), the projection algorithm (dotted), and the SMACOF algorithm (dashed) for the Alien. The number of objects $n$ is shown along the $x$-axis and the space and time requirements are shown along the y-axis. . . . . .

4.8 The Figure shows the swiss roll and its embeddings. Figure (a): swiss roll with $n=500$ vertices. Figure (b): embedding computed by the embedding algorithm. Figure (c): embedding computed by projection algorithm. Figure (d): embedding computed by SMACOF. Note that the results shown in (b)-(d) are similar except for rotations. . . . . .

4.9 Alien model used for evaluation of results. Figure (a) shows the original model, Figure (b) shows the embedding using the projection algorithm, Figure (c) shows the embedding using the embedding algorithm, and Figure (d) shows the embedding using SMACOF algorithm. . . . . . .

5.1 Models of alien in postures (a) to (c) with known ground truth. The first row shows the models, the second row shows the sample points used to find the coarse correspondence, the third row shows the canonical forms of the sample points, and the fourth row shows the color-coded correspondence. . . . . . . . . . . . . . . .

5.2 Histogram of errors made by correspondence algorithm. The black columns show the histogram of errors when corresponding posture (a) to posture (b). The grey columns show the histogram of errors when corresponding posture (a) to posture (c). . . . . . . . . . . . 
5.3 Erroneous correspondence due to symmetry problems. . . . . . . . . . 85

5.4 Models of teddy bears from the McGill 3D shape benchmark. . . . . . 85

5.5 Optimal rigid alignment of canonical forms. . . . . . . . . . . . 86

5.6 Fine correspondence computed by the algorithm. . . . . . . . . . . 87

5.7 Models of CAESAR data base. . . . . . . . . . . . . . . . 88

5.8 Modified models of CAESAR data base. . . . . . . . . . . . . . . 89

5.9 Fine correspondence computed by the algorithm. . . . . . . . . . . 90

6.1 The shape space $\mathcal{S}$ of a triangle. . . . . . . . . . . . . 95

6.2 A mesh $M$ with its dual graph $D(M) \ldots \ldots$. . . . . . . . . . . . . 95

6.3 Example of most isometric morph between two skeleton poses. Input poses are shown in (a) and (c) and morph for $t=0.5$ is shown in (b). 100

6.4 Example of isometric triangular meshes where intermediate poses interpolating all normals and edge lengths do not exist. . . . . . . . . . 101

6.5 A mesh $M$ with its dual minimum spanning tree $T(M)$. . . . . . . . 102

6.6 Illustration of how to bound the number of candidate coordinates of $v$ computed using the path through $D(e) . \ldots . \ldots 103$

6.7 Isometric morph of a simple polygon. The start polygon is a $3 D$ polygon obtained by discretizing the curve $y=\sin (x)$ and by adding thickness to the curve along the $z$-direction. The end polygon is similarly obtained from $y=-\sin x \ldots \ldots \ldots \ldots$. . . . . . . . . . . . . . . . . .

6.8 Isometric morph of a simple polygon from pose (a) to pose (i). Intermediate poses obtained using the polygon algorithm are given from left to right. . . . . . . . . . . . . . . . 106

6.9 Most isometric morph of a cycle from pose (a) to pose (i). Intermediate poses obtained using the exponential algorithm are given from left to right.107

6.10 Most isometric morph of the armadillo model from pose (a) to pose (c) and from pose (d) to pose ( $f$ ). The intermediate poses obtained using the averaging algorithm for $t=0.5$ are given in figures (b) and (e). . 108

xii 
6.11 Most isometric morph of a human from pose (a) to pose (i). Intermediate poses obtained using the averaging algorithm are given from left to right. . . . . . . . . . . . . . . . . . 109

6.12 Most isometric morph of a head from pose (a) to pose (i). Intermediate poses obtained using the averaging algorithm are given from left to right.109

6.13 The two rows show start and end poses on the left and right, respectively. The intermediate poses shown in the middle columns are interpolations for $t=0.5$ obtained using the averaging algorithm. . . . . . . . 110

6.14 Near-rigid segmentation of the armadillo and the horse model. . . . . 114

7.1 The distribution of the entries of the matrix $C$ over all $2^{k}$ candidate correspondences for one of the Alien models. The left side shows the distribution. The right side shows the Gaussian obtained by mirroring the values along $x=1 \ldots \ldots \ldots 118$

7.2 Color-coded correspondence for models of alien in postures (a) to (d) with known ground truth. . . . . . . . . . . . . . . . . . . . . . . . . . 122

7.3 Histogram of the errors made by the correspondence algorithm. . . . . 123

7.4 Color-coded correspondence for cat and gorilla models with large nonrigid deformation. . . . . . . . . . . . . . . . . . . . . . 123

7.5 Color-coded correspondence for incomplete models of a human and a bear.124

7.6 Color-coded correspondence for models of a human that do not deform isometrically. The wrong correspondence is found. . . . . . . . 125

7.7 Color-coded correspondence for models of a dancer that does not deform isometrically. . . . . . . . . . . . . . . . . 126

7.8 Application to morphing. (a): Original model. (c) Original model (grey) and correspondence (blue). (b) Morph between the models (a) and $(c) . \ldots \ldots \ldots \ldots 127$

7.9 Near-rigid segmentation of the cat model. . . . . . . . . . . . . . 127 


\section{Chapter 1}

\section{Introduction}

It is interesting thus to follow the intellectual truths of analysis in the phenomena of nature. This correspondence, of which the system of the world will offer us numerous examples, makes one of the greatest charms attached to mathematical speculations.

PierRe-Simon LAPLACE (1749-1827)

Laser-range scanners allow the accurate acquisition of the surface of real-world objects. The result of the scan is a set of three-dimensional coordinates. Various software packages exist to obtain a possibly incomplete triangulation from the set of points. More recently, it is possible to scan the surfaces of non-rigid objects such as human bodies or animals in different poses. The resulting datasets allow us to study the intrinsic shape structure from its variations. It also poses new geometric processing challenges where an important problem is to relate shapes in a consistent way.

The goal of this thesis is to find dense point-to-point correspondences between the given scans in a fully automatic way. We can solve this problem by repeatedly finding the correspondences between two of the deformed surfaces. Finding dense point-topoint correspondences between two deformed surfaces is a key problem in various applications such as mesh deformation and animation [5], shape registration [69], object recognition [49], mesh parameterization [61,89], and shape analysis [31]. The following paragraphs give a more formal problem statement. 
This thesis considers matching and morphing isometric triangular manifolds. A triangular manifold represents a shape $S$ by a triangular mesh that has the properties that each edge of $S$ is adjacent to at most two triangles and that the triangles incident on an arbitrary vertex of $S$ can be ordered locally around the vertex. Triangular manifolds are commonly used to represent shapes. Note that $S$ may contain holes and even be disconnected. The triangular manifold is a metric space where lengths can be measured as Euclidean lengths of shortest paths on that manifold. These lengths are called geodesic distances. A transformation of the manifold in $\mathbb{R}^{3}$ is an isometry if all the geodesic distances are preserved. The two transformed manifolds are then said to be isometric. Any mapping that is isometric preserves the intrinsic geometry of the manifold, that is all lengths, angles, and areas measured on the manifold [36].

Given two incomplete triangular manifolds $S^{(0)}$ and $S^{(1)}$ corresponding to two different postures of the same non-rigid object, we aim to find dense point-to-point correspondences between two deformed surfaces in a fully automatic way. That is, given a position $x^{(0)}$ on $S^{(0)}$, we aim to find the position $x^{(1)}$ on $S^{(1)}$ that corresponds to the same intrinsic location on $S^{(1)}$ as does $x^{(0)}$ on $S^{(0)}$. If the position $x^{(1)}$ is absent on $S^{(1)}$ due to incomplete data, no correspondence is found for $x^{(0)}$. This problem is known under the names of correspondence problem, registration problem, and matching problem. The problem can be viewed as a parameterization problem, where the goal is to parameterize $S^{(0)}$ onto the domain mesh $S^{(1)}$ [94].

The $3 \mathrm{D}$ models used in object recognition and shape analysis usually come from digitizing real-world objects from a discrete set of measurements using a 3D laser-range scanner or image-based reconstruction. Therefore, the reconstructed surfaces are often noisy and incomplete. This makes correspondence a challenging problem. In all of our algorithms, we assume that the input is triangulated.

The main difficulty in finding point-to-point correspondences, however, is that local regions on the surface are often not distinctive. Hence, finding the correspondence for all object points amounts to searching a large set of candidate correspondences. Previous methods to find point-to-point correspondences for deformable surfaces either restrict the search space using prior knowledge about the objects being registered [2] or use probabilistic methods to solve the problem [4], which has the drawback of 
producing inaccurate correspondences.

To solve the correspondence problem, we model the deformation of $S^{(0)}$ to $S^{(1)}$ as an isometry and study the intrinsic geometry of $S^{(0)}$ and $S^{(1)}$. Modeling deformations as isometries is appropriate, because many naturally occurring deformations are approximately isometric, for instance large-scale locomotions of human beings and animals [17]. Our approach matches the intrinsic geometries of $S^{(0)}$ and $S^{(1)}$. First, the intrinsic geometries of $S^{(0)}$ and $S^{(1)}$ are embedded into a low-dimensional Euclidean space via multi-dimensional scaling. Second, the embeddings are matched using a rigid alignment algorithm. This yields several candidate correspondences. Third, the best candidate correspondence is found based on a deformation cost.

\section{Summary of Results}

Chapter 3 To examine the intrinsic geometry of a triangular manifold $S$ with partially missing data, we need to compute geodesic distances on $S$. Chapter 3 presents an algorithm to compute approximate geodesic distances on $S$, where $S$ contains $n$ vertices. The proposed method computes an approximation of the geodesic distance between two vertices $p_{i}$ and $p_{j}$ on $S$ and provides a maximum relative error bound of the approximation. The upper bound is shown to be worst-case optimal. The algorithm approximates the geodesic distance without trying to reconstruct the missing data by embedding the surface in a low dimensional space via multi-dimensional scaling (MDS). We derive a new method to add an object to the embedding computed via least-squares MDS.

This work was presented at the Canadian Conference on Computational Geometry 2007 [9]. Note that the authors are sorted alphabetically. I was the main contributor to this paper.

A problem arising when embedding the surface in a low dimensional space via MDS in Chapter 3 is that computing the embedding via least-squares MDS [14] takes quadratic space. This limits the practical application of MDS to small data sets for pragmatic reasons. In Chapter 3, we bypass this problem by computing an embedding of an automatically computed sample set of $S$. 
Chapter 4 In Chapter 4, we consider computing the distance preserving graph embedding problem using linear space. The distance preserving graph embedding problem is to embed the vertices of a given weighted graph onto points in $d$ dimensional Euclidean space for a constant $d$ such that for each edge the distance between their corresponding endpoints is as close to the weight of the edge as possible. If the given graph is complete, that is, if the distance constraints are given as a full matrix, then multi-dimensional scaling can minimize the sum of squared embedding errors in polynomial time as outlined in Chapter 3. A serious disadvantage to this approach is its quadratic space requirement. In Chapter 4 we develop a linear-space algorithm to solve the embedding problem. A key idea is to partition a set of $n$ objects into $O(\sqrt{n})$ disjoint subsets (clusters) of size $O(\sqrt{n})$ such that the minimum inter cluster distance is maximized among all possible such partitions. Experimental results and applications to canonical representations of manifold meshes are included.

This work was presented at the Canadian Conference on Computational Geometry 2007 [7] and accepted to Computational Geometry: Theory and Applications [8]. Note that the authors are sorted alphabetically. The main contribution to this paper was achieved in close collaboration between Tetsuo Asano and myself.

Chapter 5 Chapters 3 and 4 allow us to examine the geodesic distances on a triangular manifold $S$ containing $n$ vertices with partially missing data. That is, we can study the intrinsic geometry of a surface $S$ represented by an incomplete triangular mesh. We use this fact in Chapter 5 to find dense point-to-point correspondences between two deformed surfaces corresponding to different postures of the same non-rigid object in a fully automatic way, where we model the deformations of an object as isometries. The approach requires no prior knowledge about the shapes being registered or the initial alignment of the shapes. To solve the correspondence problem, our approach maps the intrinsic geometries of the surfaces into a low-dimensional Euclidean space via multi-dimensional scaling. This results in posture-invariant shapes that can be registered using rigid correspondence algorithms. 
This work is published in the International Journal of Shape Modeling [110]. One remaining drawback of this method is that near-symmetric shapes may be registered incorrectly.

Chapter 6 We aim to overcome the problem of symmetric misalignments by choosing a correspondence that has lowest deformation cost. To achieve this, we need to take a closer look at shape deformations. Chapter 6 therefore considers morphing and presents a novel approach to morph between two isometric poses of the same non-rigid object given as triangular meshes. We model the morphs as linear interpolations in a suitable shape space $\mathcal{S}$. For triangulated $3 D$ polygons, we prove that interpolating linearly in this shape space corresponds to the most isometric morph in $\mathbb{R}^{3}$.

This work was presented at the Canadian Conference on Computational Geometry 2008 [15]. Note that the authors are sorted alphabetically. I was the main contributor to this paper. Furthermore, this work was submitted to the International Journal of Computational Geometry and Applications [108].

This shape space can be used to segment a given set of deformed surfaces into near-rigid components as summarized in Section 6.7. This part of the work was submitted to The Visual Computer [109].

Chapter 7 Chapter 7 combines the approaches of Chapters 5 and 6 to overcome the problem of symmetric misalignments. Unlike other recent methods that find the point-to-point correspondences in a fully automatic way by minimizing a deformation cost $[45,116]$, our approach does not require any user-specified parameters.

This work was submitted to the IEEE International Conference on Computer Vision 2009 [111].

The theoretical results presented in this thesis were implemented and tested on artificial data sets and on data sets obtained from a laser-range scanner. (The implementation is in $\mathrm{C}++$ and all of the experiments were conducted on an Intel Pentium D with 3.5 GB of RAM.)

At the end of each chapter, we draw conclusions. Chapter 8 summarizes the main conclusion and gives ideas for future work. 


\section{Chapter 2}

\section{Related Work}

The knowledge of which geometry aims is the knowledge of the eternal. Plato (CA 429-347 BC)

This chapter reviews previous work related to the correspondence problem and to deforming and morphing triangular meshes. Furthermore, this chapter reviews three methods that are used extensively in this thesis: multi-dimensional scaling, the fast marching method, and Voronoi sampling.

\subsection{Correspondence Problem}

This section reviews previous work aiming to find dense point-to-point correspondences between two deformed surfaces corresponding to different postures. Finding dense point-to-point correspondences has various applications in computer graphics and computer animation [5] and in statistical shape analysis [31]. Assume the point-topoint correspondences between a set of meshes are known. In computer animation, we can morph one mesh into another continuously. In statistical shape analysis, we can examine the shape variations present in the given set of shapes.

The problem of finding the correspondence between two triangular meshes has only received attention recently. Originally, a two-dimensional correspondence problem for images was studied in the field of computer vision. Given two input images of the same scene taken at different times or under different conditions, the aim of image 
registration is to find a meaningful correspondence between the two images. The image registration problem is well-studied, see Zitova and Flusser [120] for a survey. Most approaches proceed by finding and corresponding feature points in the image.

In the field of medical imaging, a three-dimensional version of the correspondence problem was studied. Given two three-dimensional input images of the same scene taken at different times or under different conditions, the aim is to find a meaningful correspondence between the two images. Maintz and Viergever [75] give an overview of techniques to solve this problem.

When considering the two- and three-dimensional image registration problems, the input images are given as height functions over a regularly sampled domain. Triangular surfaces in $\mathbb{R}^{3}$ are significantly different from images, since they are not sampled over a regular domain. Turning a mesh into a three-dimensional image using a voxelization technique significantly increases the complexity of the mesh; instead of a two-dimensional surface, the mesh becomes a discretized three-dimensional grid. Therefore, the methods developed to register two- and three-dimensional images cannot directly be applied to register surfaces.

Finding the dense point-to-point correspondences between two meshes is a special instance of the parameterization problem. The general parameterization problem aims to create piecewise mappings between triangular meshes and domains, such as planar regions or other triangular meshes. The parameterization problem is well studied; refer to Sheffer et al. [94] for a recent survey on the topic. In the special case where the domain is chosen to be one of the input meshes, the parameterization problem becomes the correspondence problem. Therefore, the correspondence problem is called cross-parameterization problem by some authors.

We consider the problem of finding point-to-point correspondences between two input meshes in a fully automatic way. The two input meshes correspond to two postures of the same model obtained by possibly non-rigid deformations. Solving this problem is known to be hard because local regions on the surface are often not distinctive. This means that finding the correspondence for all object points corresponds to searching a large set of candidate correspondences. Furthermore, the task is complicated by noisy and incomplete triangular input meshes. We review the 
most relevant work in the following.

Allen et al. [2] deform a known template mesh of a generic human body to fit a range scan of a human body. The deformation is guided by a small set of known marker positions on the object and it is ensured that the deformation is smooth in the neighborhood of each vertex. While this method performs well, a template model as well as a sparse set of marker positions need to be available.

Recently, markerless registration methods have received considerable attention [4, 20,33,50]. Anguelov et al. [4] model the registration problem using a Markov network. Two surfaces are registered by maximizing a joint probabilistic model over all correspondences. The method aims to preserve geodesic distances on the surface. Furthermore, the method ensures that close-by points in one surface map to close-by points in the other surface. This method encounters problems when registering surfaces of a human body due to symmetry alignments.

Eckstein et al. [33] propose an approach to match shapes based on surface flows. Generalized gradient flows are developed and applied to embed prior knowledge on the shapes being matched. In particular, a quasi-isometric prior is presented.

Jain et al. [50] and Bronstein et al. [20] solve the non-rigid correspondence problem by embedding the intrinsic geometry of the surface into a suitable embedding space using MDS. Denote the two surfaces being registered by $S$ and $Q$. Jain et al. embed the intrinsic geometries of both $S$ and $Q$ into Euclidean space using classical MDS [39]. MDS aims to embed the points on $S$ and $Q$, respectively, into Euclidean space $\mathbb{R}^{k}$, such that the geodesic distances on $S$ and $Q$, respectively, are approximated well by the Euclidean distances between the corresponding embedded points. The embedded surfaces are then aligned in embedding space, which yields a one-to-one correspondence between $S$ and $Q$. This fully automatic approach does not assume a template mesh or marker positions to be known. The approach was shown to perform well for surfaces with intrinsic geometry that can be represented well in Euclidean spaces. For surfaces with non-Euclidean intrinsic geometry, symmetry alignment problems may arise. The main drawback of this approach is its quadratic time and space complexity that restricts the use of this method to small models. The experiments of Jain et al. compute correspondences for models containing between 180 and 250 vertices. 
Bronstein et al. embed the intrinsic geometry of $S$ into the surface $Q$ using generalized MDS [18]. The approach also employs a coarse-to-fine strategy to overcome the high time and space complexity of the embedding problem. Generalized MDS aims to embed the points on $S$ into the surface $Q$, such that the geodesic distance on $S$ is approximated well by the geodesic distance of the corresponding points on $Q$. This method avoids the large embedding errors caused by embedding into Euclidean space. Bronstein et al. show that the method performs well in practice. The method was tested on data sets of up to 3000 vertices obtained by coarsening larger data sets $[106]$.

Bronstein et al. point out that the method is suitable for registration of incomplete surfaces. However, note that this claim is correct only if one of the surfaces to be registered is complete. If $S$ is an incomplete surface and $Q$ is a complete surface, the geodesic distances on $S$ can be weighted. However, consider the registration of two incomplete surfaces $S$ and $Q$. In order to embed the intrinsic geometry of $S$ into the surface $Q$, the generalized MDS algorithm repeatedly computes geodesic distances on $Q$. These distances are taken to be accurate by the generalized MDS algorithm. If the surface $Q$ is incomplete, this results in large embedding errors. Hence, to use the algorithm by Bronstein et al., at least one of the surfaces to be registered needs to be almost complete to avoid large embedding errors. When the aim is to register two incomplete surfaces, a template mesh is required. However, the approach by Bronstein et al. does not require prior knowledge about marker positions. Bronstein et al. [21] use a combination of generalized MDS and a rigid alignment procedure to compute a distance between two shapes. This method combines the intrinsic and extrinsic geometry of the shapes and is both bending invariant and invariant to topological noise.

Recently Huang et al. [45] and Zhang et al. [116] independently developed two similar approaches to solve the non-rigid correspondence problem. Both approaches find the correspondence by minimizing a deformation energy. Huang et al. [45] propose a technique that solves the correspondence problem iteratively by alternating between a correspondence optimization and a deformation optimization. The approach can be viewed as an extension of the Iterative Closest Point algorithm (ICP) [13] that 
is often used to solve the rigid correspondence problem. The method is shown to perform well if the two meshes are initially well aligned. If the alignment is poor, the method fails. The main drawback of this method is that it relies heavily on non-intuitive user-defined parameters. This makes the method impractical. Zhang et al. [116] propose a technique that solves the correspondence problem by finding a small set of features and by choosing the best feature correspondence as the one that minimizes a deformation energy. To improve the efficiency of the algorithm, the tree of all matching features is pruned if the features are too dissimilar. Nonetheless, the algorithm is not as efficient as the algorithm of Huang et al. [45]. Once the feature correspondences are computed, the full correspondence is found by deforming the full mesh based on the feature points. The main drawback of this method is the computational inefficiency. Results are only demonstrated for models with less than 4000 vertices. Furthermore, like the method of Huang et al., the tree pruning relies heavily on non-intuitive user-defined parameters. The authors leave finding parameters automatically for future work.

\subsection{Morphing}

Morphing aims to compute a smooth deformation between a source shape and a target shape in two or three dimensions. We consider morphing between two triangular manifold meshes in three dimensions. To solve this problem, we need to solve two sub-problems. First, we need to establish a meaningful correspondence between the two shapes as described in Section 2.1. Second, we need to find smooth paths that are followed by the vertices of the mesh. Since work solving the first task is reviewed in the previous section, this section focuses on work solving the second task.

Computing a smooth morph from one pose of a shape in two or three dimensions to another pose of the same shape has numerous applications. For example in computer graphics and computer animation this problem has received considerable attention [3,65]. A recent survey on this topic was written by Alexa [1]. We only review the work most relevant to this thesis.

Before considering morphing three-dimensional mesh models, the two-dimensional 
version of the problem has received considerable attention. In case where the input is sampled over a regular domain, this problem is called image morphing. Imagemorphing is widely studied and surveyed by Wolberg [107]. In case where the input is sampled over an irregular domain, the problem becomes to interpolate between two simple polygons in the plane. We only review work that makes use of intrinsic representations of the polygons. Sederberg et al. [90] propose to interpolate an intrinsic representation of two-dimensional polygons, namely the edge lengths and interior angles of the polygon. Surazhsky and Gotsman [101] morph by computing mean value barycentric coordinates based on an intrinsic representation of triangulated polygons. This method is guaranteed to be intersection free. Iben et al. [46] morph planar polygons while guaranteeing that no self-intersections occur using an approach based on energy minimization. This approach can be constrained to be as isometric as possible.

For three-dimensional models, physically based methods were traditionally used to achieve mesh morphing or mesh deformation. These approaches deform triangular meshes by first assigning a skeleton to the mesh. Second, each vertex on the mesh is assigned to one or multiple bones. This step is called skinning or skin animation. Finally, the skeleton is deformed and the skin follows the skeleton based on the given weights. If only few input meshes are available, the bones can be automatically computed using Reeb graphs [105]. If a set of meshes is available that represents the deformation space of the given object, bones can automatically be computed by fitting bones to near-rigid clusters of triangles [51]. James and Twigg [51] then compute skinning parameters and add corrections progressively. This is similar to the skeleton-driven deformation presented by Lewis et al. [68].

Alternatively, three-dimensional models can be deformed using approaches that operate on the surface directly. Sun et al. [100] morph between three-dimensional manifold meshes. They extend the approach by Sederberg et al. to three dimensions by extending the intrinsic representation to polyhedra. However, the developed methods are computationally expensive [1]. Another extension of the approach by Sederberg et al. [90] to three dimensions is presented by Lipman et al. [72]. They give a rotation-invariant mesh representation that is similar in spirit to the first and 
second fundamental form in differential geometry. This representation can be used to find an approximate morph. No energy minimization is required in this work. Kraevoy and Sheffer [60] present a similar representation that is based on mean-value encodings of vertices. This representation has the property that similar models have similar encodings. The representation can be used for morphing. Morphing requires minimizing a non-linear energy function.

Sorkine and Alexa [98] propose an algorithm to deform a surface based on a given triangular surface and updated positions of few feature points. The surface is modeled as being covered by overlapping cells. The deformation aims to deform each cell as rigidly as possible. The overlap is necessary to avoid stretching along cell boundaries. The deformation is based on minimizing a global non-linear energy function that is simple to implement. The energy is guaranteed to converge. However, since the energy function may have multiple minima, the algorithm is not guaranteed to find the global minimum. The approach tends to preserve the edge lengths of the triangular mesh. This property depends upon finding a global minimum of the energy function. One cannot guarantee to find this global minimum.

Recently, Kilian et al. [55] used shape space representations to guide morphs and other more general deformations between shapes represented as triangular meshes. Each shape is represented by a point in a high-dimensional shape space and deformations are modeled as geodesics in shape space. The geodesic paths in shape space are found using an energy-minimization approach. Before Kilian et al. [55] presented the use of a shape space for shape deformation and exploration of triangular meshes, shape space representations were developed to deform shapes in different representations. Cheng et al. [26] proposed an approach that deforms shapes given in skin representation, which is a union of spheres that are connected via blending patches of hyperboloids, with the help of a suitable shape space. Furthermore, algorithms for deforming curves with the help of shape space representations were proposed by Younes [115] and Klassen et al. [58]. Eckstein et al. [33] propose a generalized gradient descent method similar to the approach by Kilian et al. that can be applied to deform triangular meshes. All of these approaches depend on solving a highly non-linear optimization problem with many unknown variables using numerical solvers. It is 
therefore not guaranteed that the globally optimal solution is found.

\subsection{Multi-Dimensional Scaling}

MDS is a commonly used technique to reduce the dimensionality of high-dimensional data. Given a set of $n$ objects $O_{1}, \ldots, O_{n}$ in $d$ dimensions as well as the pairwise dissimilarities $\delta_{i, j}, 1 \leq i, j \leq n$ with $\delta_{i, j}=\delta_{j, i}$ between objects $O_{i}$ and $O_{j}$, the aim is to find points $X_{1}, \ldots, X_{n}$ in a $k$-dimensional space with $k<d$, such that the distance $d_{i, j}(X)$ between $X_{i}$ and $X_{j}$ equals $\delta_{i, j}$ for $1 \leq i, j \leq n$. The distances do not necessarily need to be Euclidean distances.

Metric MDS aims to find a set of embeddings, such that $d_{i, j}(X)=f\left(\delta_{i, j}\right)$, where $f$ is a continuous parametric monotonic function. For a thorough review of metric MDS methods, refer to [28]. Metric MDS can be viewed as a mapping from arbitrary objects $O_{i}$ in $d$ dimensions to points $X_{i}$ in $k$ dimensions with the constraint that an objective function $E$ is minimized.

Nonmetric MDS also aims to find a set of embeddings, such that $d_{i, j}(X)=f\left(\delta_{i, j}\right)$, but abandons the metric nature of the transformation function $f$. The function $f$ can now be an arbitrary monotone function. That is, only the rank order of the dissimilarities needs to be preserved. We do not review nonmetric MDS in this thesis, since in our applications, we aim to preserve more than the rank.

We consider metric MDS in case where $f$ is the identity function. This aim can be shown to be too ambitious, since in general it is not possible to find positions $X_{1}, \ldots, X_{n}$ in $k$ dimensions such that $d_{i, j}(X)=\delta_{i, j}$ for all $i, j$. To find a good approximation, different related optimality measures can be used. In the following, the main two ways to compute MDS are reviewed [28]. Classical MDS, also called principal coordinate analysis, is a method closely related to Principal Component Analysis that assumes that the dissimilarities are Euclidean distances in a high dimensional space and that aims to minimize $E_{P C O}=\sum_{i=1}^{n} \sum_{j=i+1}^{n}\left(\delta_{i, j}^{2}-d_{i, j}(X)^{2}\right)$ by finding a mapping as eigenvectors of a matrix. Least-squares MDS aims to minimize $E_{L S}=\sum_{i=1}^{n} \sum_{j=i+1}^{n} \omega_{i, j}\left(\delta_{i, j}-d_{i, j}(X)\right)^{2}$, where $\omega_{i, j}$ is a non-negative weight that can be viewed as a confidence value corresponding to the dissimilarity $\delta_{i, j}$. 


\subsubsection{Classical Multi-Dimensional Scaling}

Classical MDS was introduced by Gower [39]. It aims to find points $X_{i}$ in $k$ dimensions, such that

$$
E_{P C O}=\sum_{i=1}^{n} \sum_{j=i+1}^{n}\left(\delta_{i, j}^{2}-d_{i, j}(X)^{2}\right)
$$

is minimized. Denote the position vector of point $X_{i}$ by $\vec{x}_{i}=\left[\begin{array}{llll}x_{i, 1} & x_{i, 2} & \ldots & x_{i, k}\end{array}\right]^{T}$ and denote the point matrix by $X=\left[\begin{array}{llll}\vec{x}_{1} & \vec{x}_{2} & \ldots & \vec{x}_{n}\end{array}\right]^{T}$. Let $B=X X^{T}$ be the inner product matrix of $X$. Note that $B$ is denoted inner product matrix in the literature although $B$ is the outer product of the matrix $X$. However, the entries of $B$ are the pairwise inner products of the vectors $\vec{x}_{i}$.

The goal is to find the matrix $X$. Note that the quality of the point set $X$ is invariant with respect to translations, rotations, and reflections. Therefore, we can choose that the centroid of the point set is at the origin of the coordinate system, that is, $\sum_{r=1}^{n} x_{r, i}=0, i=1, \ldots, n$. This yields $B=H A H$, where $A$ is a matrix with elements $A_{i, j}=-\frac{1}{2} d_{i, j}(X)^{2}, 1 \leq i, j \leq n$ and where $H=I-\frac{1}{n} 1$ with $I$ as $n \times n$ identity matrix and 1 as $n \times n$ matrix containing 1 at each position [28, p.33]. Note that the right hand side of this equation is known when we use the optimal case $d_{i, j}(X)=\delta_{i, j}, 1 \leq i, j \leq n$. This allows us to compute the product matrix $B$.

Note that $B$ is a symmetric positive semi-definite matrix, since it was obtained using symmetric and non-negative distance measures. Hence, a spectral decomposition can be performed on $B$. Let $B=V \Lambda V^{T}$ be the spectral decomposition with sorted eigenvalues in decreasing order, where $V$ is the matrix of eigenvectors of $B$ and $\Lambda$ is the diagonal matrix of eigenvalues of $B$. Then $X=V \Lambda^{\frac{1}{2}}$ yields the sought points. A practical algorithm that takes the target dimension into account and does not compute the full spectral decomposition of $B$ is given by Cox and Cox [28, p.38-39]. Classical MDS minimizes $E_{P C O}=\sum_{i=1}^{n} \sum_{j=i+1}^{n}\left(\delta_{i, j}^{2}-d_{i, j}(X)^{2}\right)$ if $\delta_{i, j}$ are Euclidean distances in a high-dimensional space. This algorithm takes $O\left(k n^{2}\right)$ time and $O\left(n^{2}\right)$ space [63].

One disadvantage of this method is that the objective function $E_{P C O}$ aims to minimize a Frobenius norm and is not meaningful if the dissimilarities cannot be viewed as Euclidean distances in a high dimensional space. Further, no weights or confidence values can be assigned to the dissimilarities between objects. The main 
advantage of classical MDS is that the solution cannot get stuck in local minima.

\subsubsection{Least-Squares Multi-Dimensional Scaling}

Least-squares MDS aims to find points $X_{i}$ in $k$ dimensions, such that

$$
E_{L S}=\sum_{i=1}^{n} \sum_{j=i+1}^{n} \omega_{i, j}\left(\delta_{i, j}-d_{i, j}(X)\right)^{2}
$$

is minimized, where $\omega_{i, j}$ are non-negative weighting coefficients with $\omega_{i, j}=\omega_{j, i}$. Since the objective function $E_{L S}$ is a complex function, it is easier to iteratively approximate the objective function by a simple function. This approach is pursued in the algorithm Scaling by Maximizing a Convex Function (SMACOF) that is explained by Borg and Groenen [14, p.146-155] and used by Elad and Kimmel [34] to compute canonical forms. SMACOF proceeds by iteratively refining a simple majorization function that bounds the objective function $E_{L S}$ from above.

We can rewrite the objective function [14] as

$$
E_{L S}=\alpha+\beta-\gamma
$$

with

$$
\alpha=\sum_{i=1}^{n} \sum_{j=i+1}^{n} \omega_{i, j} \delta_{i, j}^{2}, \beta=\sum_{i=1}^{n} \sum_{j=i+1}^{n} \omega_{i, j} d_{i, j}(X)^{2} \text { and } \gamma=2 \sum_{i=1}^{n} \sum_{j=i+1}^{n} \omega_{i, j} d_{i, j}(X) \delta_{i, j}
$$

and using the Cauchy-Schwartz inequality bound it by

$$
E_{L S} \leq \sum_{i=1}^{n} \sum_{j=i+1}^{n} \omega_{i, j} \delta_{i, j}^{2}+\operatorname{tr}\left(X^{T} V X\right)-2 \operatorname{tr}\left(X^{T} B(Z) Z\right)=: \tau
$$

where $V$ is an $n \times n$ matrix with elements

$$
V_{i, j}=\left\{\begin{array}{l}
-\omega_{i, j} \text { if } i \neq j \\
\sum_{i \neq r} \omega_{i, r} \text { if } i=j
\end{array}\right.
$$


with $e_{i}$ as $i$ th column of the $n \times n$ identity matrix, $Z$ is a possible solution for $X$, and $B(Z)$ is an $n \times n$ matrix with elements

$$
B_{i, j}=\left\{\begin{array}{l}
-\frac{\omega_{i, j} \delta_{i, j}}{d_{i, j}(Z)} \text { if } i \neq j, d_{i, j}(Z) \neq 0 \\
0 \text { if } i \neq j, d_{i, j}(Z)=0 \\
\sum_{l=1, l \neq i}^{n} B_{i, l} \text { if } i=j
\end{array} .\right.
$$

The minimum of $\tau$ can be found by setting the gradient of $\tau$ to zero. Setting the gradient to zero yields an iterative approach to update the current solution of $X$ using the formula $X_{r+1}=V^{+} B\left(X_{r}\right) X_{r}$, where $X_{r}$ is the position of $X$ after $r$ iterations and $V^{+}$is the pseudo-inverse of $V$. The initial configuration $X_{0}$ can be chosen as a random point set. It can be shown that this approach of solving the optimization problem is equivalent to using a gradient descent method to minimize $E_{L S}$ and therefore has only a linear convergence rate. Furthermore, each iteration requires the inversion of an $n \times n$ matrix. The algorithm takes $O\left(n^{2} t\right)$ time, where $t$ is the number of iterations needed until convergence of $X$.

We minimize $\tau$ using a quasi-Newton method instead. The quasi-Newton method used is the limited-memory Broyden-Fletcher-Goldfarb-Shanno scheme [73]. This quasi-Newton method offers the advantage of obtaining close to quadratic convergence rates without the need to compute the inverse of the Hessian matrix explicitly in each step. Instead LSBFGS updates an approximation to the inverse Hessian matrix in each iterative step, such that the approximation converges to the true inverse of the Hessian in the limit. Using LSBFGS instead of a simple gradient descent method to minimize $\tau$ reduced the number of iterations $t$ required until convergence significantly in our experiments.

The main disadvantage of this method is that the optimization can get stuck in local minima. However, Elad and Kimmel [34] found that this method is more accurate in practice than classical MDS.

The recent approach of anchored MDS [23] enhances MDS by allowing to embed groups of objects in the following way. The data is interactively divided into two groups, a group of objects called anchors, and a group of objects called floaters. The anchors either have fixed coordinates or are embedded using MDS. The floaters are 
then embedded with respect to the anchors only. That is, anchors affect the embedding of floaters, but floaters do not affect the embedding of anchors.

\subsubsection{Multi-Dimensional Scaling in Geometry Processing}

Elad and Kimmel [34] applied an MDS technique to the problem of matching threedimensional objects. Geodesic distances on the object are used as dissimilarities and MDS is applied. The resulting shapes are posture-invariant shapes and are called canonical forms by Elad and Kimmel.

More formally, Elad and Kimmel define the canonical form of a triangular manifold $S$ as the mapping of $S$ to a low-dimensional Euclidean space, such that the Euclidean distances between the mapped vertices approximate the geodesic distances between the original vertices well. The canonical form is computed via MDS with the geodesic distances between vertices on the triangular manifold as dissimilarities. This has the effect that the canonical form of a non-rigid body does not change as the body moves. That is, the canonical form is posture invariant. Elad and Kimmel use the property of posture-invariance of the canonical form to match objects in different postures.

Canonical forms were used by Bronstein et al. [16-19,22] for face recognition. Bronstein et al. [16] compute canonical forms of three-dimensional representations of faces in $\mathbb{R}^{3}$ and use these expression-invariant canonical forms for face recognition. They extend the technique to handle open and closed mouths [17]. Furthermore, multigrid MDS is introduced to achieve more efficient algorithms [22]. Bronstein et al. $[18,19]$ handle face recognition in the presence of incomplete data by embedding one face in the space of another face instead of embedding the face in $\mathbb{R}^{3}$. Jain and Zhang [49] improved the efficiency of the surface matching algorithm using canonical forms by computing an approximate canonical form via the Nyström approximation and Jain et al. [50] find one-to-one correspondences of isometric surfaces using canonical forms. 


\subsection{The Fast Marching Method}

Finding shortest paths and shortest distances between points on a surface $S$ in threedimensional space is a well-studied problem in discrete differential geometry [57] and computational geometry [77].

Different related problems on finding geodesic paths and distances depending on the number of source and destination points have been studied. We outline the three most commonly studied problems. The first problem is finding the geodesic path from one source vertex $s \in S$ to one destination vertex $d \in S$. The second problem known as single source shortest path problem is finding the geodesic paths from one source vertex $s \in S$ to all destination vertices in $S$ or equivalently finding the geodesic paths from all source vertices in $S$ to one destination vertex $d \in S$. The third problem known as all-pairs shortest path problem is finding the geodesic paths between all pairs of vertices $s$ and $d$ in $S$.

One of the most commonly used techniques in practice to compute geodesic paths on triangular meshes is the fast marching method on triangular domains [56,92]. We use the fast marching method in this thesis.

The fast marching method solves the single source shortest path problem by solving the Eikonal equation, a partial differential equation describing a wave propagation, on a triangular grid with $n$ vertices. The result is based on Sethian's method to solve the Eikonal equation on a quadrilateral grid [91]. The fast marching method is similar to Dijkstra's algorithm in that a front is propagated from the source to all vertices in $S$. However, the resulting approximation produces the correct shortest path on a regular orthogonal grid. To run the fast marching method on triangular meshes, the triangles are locally unfolded and obtuse angles are split. The algorithm's running time is $O(n \log n)$. The algorithm requires $O(n)$ space. The accuracy of the approach depends on two properties of the underlying triangulation; namely, the longest edge and the widest angle in the triangular mesh [56]. Each obtuse angle in the triangulation decreases the accuracy of the approach. As the front is propagated, inaccuracies accumulate with each obtuse angle that is encountered. Hence, the fast marching method is less accurate when the path crosses a large number of obtuse 
triangles.

Since the algorithm is easy to implement and performs well in practice, several implementations and extensions of the algorithm exist. Yatziv et al. [114] improve the running time of the fast marching technique by using an untidy priority queue. Their experimental results show that the accuracy of the computed shortest paths only suffers slightly from this newly introduced inaccuracy. Kirsanov [57] introduces a novel update rule for the fast marching technique during the march. This update rule yields a higher accuracy of the resulting shortest paths. Bertelli et al. [12] consider solving the all-pairs shortest path problem using the fast marching technique. Their goal is to take advantage of the redundant computation in different passes of the single source shortest path algorithm to obtain a more efficient approach than simply running the fast marching algorithm $n$ times with each vertex as source point. Although the algorithm is shown to achieve higher efficiency in experiments, the worst case running time remains $O\left(n^{2} \log n\right)$.

\subsection{Voronoi Sampling}

Sampling aims to compute a set $P$ of $n_{P}$ sample points on a triangular manifold $S$ that represent the overall shape of $S$ well. Voronoi sampling chooses a set of samples evenly distributed with respect to the geodesic distance on $S$.

Voronoi sampling, also called Farthest Point Sampling, allows to obtain evenly distributed samples in an iterative way. That is, the approach iteratively obtains samples and the samples are evenly distributed at every stage of the sampling approach. Voronoi sampling was introduced to image processing, or 2D signal processing, by Eldar et al. [35]. The sampling approach starts from a random sample and iteratively computes the next sample as the vertex which is farthest from the samples computed so far. If the metric is chosen to be the Euclidean distance, the new sample can be viewed as the vertex which is the center of the largest circle not containing any sample points. This observation yields an elegant relationship between the sampling approach and Voronoi diagrams that can be used to obtain an efficient sampling algorithm. Eldar et al. show that this sampling approach has favorable properties such as a high 
data acquisition rate and good anti-aliasing properties.

The Voronoi sampling approach can be extended to obtaining samples on a $3 \mathrm{D}$ surface. In this case, the distance metric to measure uniformity is the distance metric intrinsic to the surface being sampled. That is, the geodesic distance on the surface. This yields a relationship between the sampling approach and generalized Voronoi diagrams. Generalized Voronoi diagrams are Voronoi diagrams built in an arbitrary metric space. They are known to possibly have disconnected and non-convex cells, which complicates the use of Voronoi diagrams for the sampling approach. Voronoi diagrams with disconnected and non-convex cells may have large complexity and it is costly to store them explicitly. Moenning and Dodgson [79] combine the fast marching technique and Voronoi sampling to overcome the problem of storing the Voronoi diagram explicitly. The main idea is to run the fast marching technique while tracking multiple propagation fronts originating from different sample points. Moenning and Dodgson claim that the resulting algorithm takes $O(n \log n)$ time to compute $n_{P}$ uniformly distributed sample points on a surface with $n$ vertices, but the algorithm appears to take $O\left(n_{P} n \log n\right)$ time.

To perform MDS, combining the fast marching technique and Voronoi sampling to obtain samples works well in practice $[34,50]$. 


\section{Chapter 3}

\section{Approximating Geodesics}

We come now to the question: what is a priori certain or necessary, respectively in geometry (doctrine of space) or its foundations? Formerly we thought everything; nowadays we think nothing. Already the distanceconcept is logically arbitrary; there need be no things that correspond to it, even approximately.

Albert Einstein (1879-1955)

We aim to examine the intrinsic geometry of a triangular manifold $S$ to solve the correspondence problem. Hence, we need to compute geodesic distances on $S$. This chapter presents an algorithm to compute approximate geodesic distances on a triangular manifold $S$ containing $n$ vertices with partially missing data. The proposed method computes an approximation of the geodesic distance between two vertices $p_{i}$ and $p_{j}$ on $S$ and provides a maximum relative error bound of the approximation. The upper bound is shown to be worst-case optimal. The algorithm approximates the geodesic distance without trying to reconstruct the missing data by embedding the surface in a low dimensional space via multi-dimensional scaling (MDS). We derive a new method to add an object to the embedding computed via least-squares MDS. 


\subsection{Introduction}

We study the computation of geodesic distances to examine the intrinsic geometry of a triangular manifold $S$ to solve the correspondence problem. Furthermore, the computation of geodesic distances on a triangular manifold is interesting in its own right, because it is a common operation in many applications. In computer graphics, geodesic distances were recently applied to produce parameterizations with low distortion, which has direct applications to texture mapping and morphing [83,118,119]. Geodesic distances can further be applied to classify isometric shapes [34,42,49,113]. Bronstein et al. $[16,17,19,22]$ use geodesic distances for face recognition. The 3D models used in these applications usually come from digitizing real-world objects from a discrete set of measurements using a 3D laser-range scanner or image-based reconstruction. Therefore, the reconstructed surfaces are often incomplete. Despite of efforts on fixing holes in triangular meshes [29], a general reliable hole-filling algorithm is still unavailable.

In this chapter, we explore the problem of computing estimates on geodesic distances with worst-case optimal upper bounds on a triangular manifold $S$ with partially missing data without attempting to fill the holes of $S$. To our knowledge, this problem has not been explored so far. The main advantage of this approach compared to previous approaches to compute geodesic distances on triangular manifolds $[56,78,102]$ is that the error of the estimate is bounded for incomplete surfaces. The resulting approximated geodesic distances can be used to modify the above-mentioned applications for models with incomplete surface descriptions.

The approximation of the geodesic distance consists of three main steps. First, we compute the geodesic distance $\delta_{i, j}$ between the vertices $p_{i}$ and $p_{j}$ for $i, j \in P$, where $P$ is a set of indices of uniformly distributed sample points on $S$, using the fast marching technique introduced by Kimmel and Sethian [56] (see Section 2.4). Note that the geodesic path between $p_{i}$ and $p_{j}$ computed by the fast marching technique may trace a hole of the model and therefore be incorrect, see Figure 3.1. Furthermore, we compute confidence values $\omega_{i, j}=1-\frac{m_{i, j}^{h}}{m_{i, j}}$, where $m_{i, j}$ is the number of edges on the geodesic path computed by the fast marching technique from $p_{i}$ to $p_{j}$ and where $m_{i, j}^{h}$ is the 
number of edges tracing a hole of $S$ on the geodesic path from $p_{i}$ to $p_{j}$.

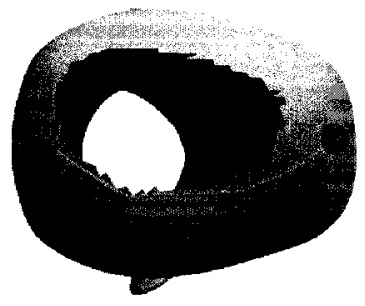

Figure 3.1: Paths computed via the fast marching technique tracing boundaries of holes.

Second, we use the geodesic distances $\delta_{i, j}$ as dissimilarities and the confidence values $\omega_{i, j}$ as weights to embed the manifold $S$ in a low-dimensional Euclidean space via multi-dimensional scaling. In this way, we obtain a canonical form of $S$ similar to the one introduced by Elad and Kimmel [34].

Third, we compute an estimate of the true geodesic distance between two arbitrary vertices $p_{i}$ and $p_{j}$ on $S$ by projecting $p_{i}$ and $p_{j}$ to the canonical form of $S$ using an extension of the technique devised by Gower [40]. The Euclidean distance between the embedded points approximates the true geodesic distance between the original points.

\subsection{Adding an Object to the Multi-Dimensional Scaling Embedding}

A question that arises in MDS is how to treat an additional object $O_{n+1}$ in $d$ dimensional space with corresponding dissimilarities $\delta_{n+1,1}, \ldots, \delta_{n+1, n}$ that becomes available only after the objects $O_{1}, \ldots, O_{n}$ have been mapped to points $X_{1}, \ldots, X_{n}$ in $k$-dimensional space. An inefficient approach to this problem is to disregard the previously computed points $X_{1}, \ldots, X_{n}$ and to apply MDS to $n+1$ objects as before. A more efficient approach to this problem for classical MDS was derived by Gower [40]. Assume that we already computed the $n \times k$ matrix $X$ containing the positions of the first $n$ points $X_{1}, \ldots, X_{n}$. To find the embedding $X_{n+1}$ of the object $O_{n+1}$ in $k$-dimensional space, we use the previously computed embedding $X$ and the dissimilarities $\delta_{n+1,1}, \ldots, \delta_{n+1, n}$ describing the relationship between $O_{n+1}$ and 
the remaining objects. Let $d_{i}^{2}$ denote the squared distance between $X_{i}, i=1, \ldots, n$ and the centroid of $X$. Since the centroid of $X$ was chosen to be the origin, $d_{i}^{2}=B_{i, i}$, where $B_{i, i}$ is the diagonal element of the previously computed matrix $B=X X^{T}$. We can compute an $n \times 1$ vector $D$ containing elements $D_{i}=d_{i}^{2}-d_{n+1, i}^{2}$ if we use the optimal case $d_{n+1, i}(X)=\delta_{n+1, i}, 1 \leq i \leq n$. The embedding $X_{n+1}$ can then be computed as

$$
X_{n+1}=\frac{1}{2}\left(X^{T} X\right)^{-1} X^{T} D .
$$

Note that the matrix $X^{T} X$ that is inverted has dimension $k \times k$ and that the inverse is therefore fast to compute in typical applications (with $k=2$ or $k=3$ ). Gower proved that the position of $X_{n+1}$ computed using this method is identical to the position of $X_{n+1}$ when classical MDS is performed for all the $n+1$ objects at once.

To add an object $O_{n+1}$ to the least-squares MDS embedding, we are also given the corresponding weights $\omega_{n+1,1}, \ldots, \omega_{n+1, n}$. The technique by Gower does not yield satisfying results, since the objective function minimized for the embedding of the objects $O_{1}, \ldots, O_{n}$ is $E_{L S}$. Instead, we try to minimize the least-squares function

$$
E_{L S}^{*}=\sum_{i=1}^{n} \omega_{n+1, i}\left(\delta_{n+1, i}-d_{n+1, i}(X)\right)^{2},
$$

which can be written as

$$
E_{L S}^{*}=\alpha^{*}+\beta^{*}-\gamma^{*}
$$

where

$$
\begin{gathered}
\alpha^{*}=\sum_{i=1}^{n} \omega_{n+1, i} \delta_{n+1, i}^{2}, \beta^{*}=\sum_{i=1}^{n} \omega_{n+1, i}\left(\vec{x}_{i}-\vec{x}_{n+1}\right)^{T}\left(\vec{x}_{i}-\vec{x}_{n+1}\right), \text { and } \\
\gamma^{*}=2 \sum_{i=1}^{n} \omega_{n+1, i} \delta_{n+1, i} \sqrt{\left(\vec{x}_{i}-\vec{x}_{n+1}\right)^{T}\left(\vec{x}_{i}-\vec{x}_{n+1}\right)} .
\end{gathered}
$$

We can now compute the gradient of this objective function with respect to the point $\vec{x}_{n+1}$ analytically as

$$
\nabla E_{L S}^{*}=\sum_{i=1}^{n} 2 \omega_{n+1, i}\left(\vec{x}_{n+1}^{T}-\vec{x}_{i}^{T}\right)\left(1-\frac{\delta_{n+1, i}}{d_{n+1, i}}\right) .
$$


This allows us to add the object $O_{n+1}$ to the MDS embedding by minimizing $E_{L S}^{*}$ using a LSBFGS quasi-Newton approach.

Note that this approach can be used to compute an embedding incrementally. When all of the $n$ objects and pairwise dissimilarities are available beforehand, we can compute an embedding using least-squares MDS in $O\left(n^{2} k t\right)$ time and $O\left(n^{2}\right)$ space, where $t$ is the number of iterations of the quasi-Newton approach. If the objects become available one by one instead, we can repeatedly add an object to the embedding using the approach described above. To add object $O_{n+1}$, the approach takes $O(n k t)$ time and $O(n k)$ space, where $t$ is the number of iterations of the quasiNewton approach. Since we can reuse the space after one object was embedded, it takes $O(n k t(1+2+\ldots+n))=O\left(n^{3} k t\right)$ time and $O(n k)$ space to embed $n$ objects incrementally. That is, our incremental algorithm outperforms the least-squares MDS algorithm in terms of space. Note however, that while least-squares MDS solves an optimization problem for $k n$ unknown variables, the incremental approach fixes coordinates incrementally and repeatedly solves an optimization problem for $k$ variables only. Hence, we expect the quality of the computed embedding to be higher for least-squares MDS than for our incremental approach. In Section 4, we present an approach that uses $O(n k)$ space while yielding embeddings of high quality.

Although the authors are not aware that this addition to the least-squares MDS embedding was discussed previously, this is not the main contribution of this chapter.

\subsection{Estimating Geodesic Distances}

We use the canonical form to estimate the geodesic distance between any given pair $p_{i}$ and $p_{j}$ of vertices on a triangular manifold $S$ with partially missing data, where $1 \leq i, j \leq n$. The main idea of the approach is to compute the canonical form of the manifold based on weighted geodesic distances on $S$. That is, we use geodesic distances as dissimilarities $\delta_{i, j} \forall i, j \in S$ and we use confidence values $\omega_{i, j}=1-\frac{m_{i, j}^{h}}{m_{i, j}}$, where $m_{i, j}$ is the number of edges on the geodesic path from $p_{i}$ to $p_{j}$ on $S$ and where $m_{i, j}^{h}$ is the number of edges on the geodesic path on $S$ from $p_{i}$ to $p_{j}$ that pass through triangles of $S$ which share at least one vertex with the boundary of a hole. Since $S$ 
is a manifold, every edge not adjacent to a hole of $S$ has degree two. Hence, we can find the edges of $S$ tracing a hole of $S$ as edges of degree less than two. We chose this measure for $\omega_{i, j}$, since it can be computed more efficiently than the fraction of the length of the path that does not trace the boundary of a hole. When working with data obtained from laser range scanners, $\omega_{i, j}$ is a good approximation of the fraction of the path that does not trace the boundary of a hole, because all of the edges of $S$ have similar lengths. If paths that trace holes of $S$ obtain weight 0 , it can be proven that Euclidean distances in embedding space approximate the original geodesics well [86]. Since we wish to extrapolate information using the metric property of the manifold, we weigh distances tracing a hole of $S$ less than accurate distances, but we do not disregard those distances completely.

Let $\hat{S}$ denote the complete surface partially represented by $S$. Let $\hat{\delta}_{i, j}$ be the geodesic distance between $p_{i}$ and $p_{j}$ on $\hat{S}$ and let $\delta_{i, j}$ be the geodesic distance between $p_{i}$ and $p_{j}$ on $S$. Note that $\delta_{i, j}$ equals $\hat{\delta}_{i, j}$ if the geodesic path between $p_{i}$ and $p_{j}$ on $S$ does not trace a hole of $S$. Let $\hat{d}_{i, j}$ denote the Euclidean distance between $p_{i}$ and $p_{j}$. After computing the canonical form, $p_{i}$ is associated with a point $X_{i}$ in embedding space and $p_{j}$ is associated with a point $X_{j}$ in embedding space. As before, $d_{i, j}(X)$ denotes the Euclidean distance between $X_{i}$ and $X_{j}$ in embedding space.

Note that the geodesic distances $\hat{\delta}_{i, j}$ form a metric. That is, $\hat{\delta}_{i, j}$ is non-negative, symmetric, and satisfies the triangle inequality. If $\hat{S}=S$, the set of dissimilarities $\hat{\delta}_{i, j} \forall i, j \in S$ contains therefore redundant information. When $S$ is a true subset of $\hat{S}$, we take advantage of this redundancy by weighing well approximated geodesic distances higher than geodesic distances tracing around a hole of $S$. We use the geodesic distances with confidence values to compute a canonical form of a sample set of $S$ with indices in $P$. This sample set is necessary for objects with hundreds of thousands of vertices, since computing the canonical form is not only computationally expensive, but also requires quadratic storage in the number of vertices to embed due to the quadratic number of dissimilarities and weights. For real-life data sets, an algorithm using quadratic storage is not feasible. Taking a sample set $P$ of vertices for the embedding has a negative effect on the quality of the results however and the sample set should therefore be large enough to represent the overall shape of $S$ well. 
We use curvature-based sampling and Voronoi sampling to choose the sample set $P$. The approach is discussed in Section 3.3.1.

Note that the canonical form has the property that Euclidean distances in the canonical form approximate geodesic distances on $S$ well according to the optimality measure $\sum_{i \in P} \sum_{j \in P}\left(1-\frac{m_{i, j}^{h}}{m_{i, j}}\right)\left(\delta_{i, j}-d_{i, j}(X)\right)^{2}$. Hence, we expect $d_{i, j}$ to be a good approximation of $\hat{\delta}_{i, j}$ on $\hat{S}$ even if $\delta_{i, j}$ is obtained by a path tracing a hole of $S$. In fact, the error made by approximating $\hat{\delta}_{i, j}$ by $d_{i, j}$ can be bounded and the upper bound is worst-case optimal. The approach used to compute an error bound for the approximation is discussed in Section 3.3.2.

A detailed overview of the algorithm suggested to estimate geodesic distances on incomplete triangular manifolds is given in Section 3.4. An analysis of the algorithm is presented in Section 3.5.

\subsubsection{Sampling Strategies}

We aim to compute a set $P$ of $n_{P}$ sample points on $S$ that represent the overall shape of $S$ well. Two different sampling strategies were implemented and tested. The results show that the sampling strategy has a significant influence on the shape of the canonical form and on the accuracy of the geodesic approximation.

\section{Curvature-Based Sampling}

A set $P$ of $n_{P}$ sample points on $S$ can be obtained using curvature-based sampling. The strategy aims to maintain points in regions of high curvature and to reduce the point density in regions of low curvature. We obtain $P$ using the software package IMCompress of PolyWorks, a 3D visualization and editing software package by InnovMetric.

\section{Voronoi Sampling}

We aim to compute a set $P$ of $n_{P}$ sample points on $S$ that represent the overall shape of $S$ well. To choose a set of samples uniformly distributed with respect to the geodesic distance on $S$, we use Voronoi sampling as explained in Section 2.5. 


\subsubsection{Error Bounds of the Approximation}

The error made by approximating the geodesic distance $\hat{\delta}_{i, j}$ on the complete surface by the Euclidean distance $d_{i, j}$ between $X_{i}$ and $X_{j}$ in embedding space can be bounded by finding an upper bound and a lower bound for $\hat{\delta}_{i, j}$.

A lower bound $\hat{\delta}_{i, j}^{\text {lower }}$ on $\hat{\delta}_{i, j}$ is given by the Euclidean distance $\hat{d}_{i, j}$ between $p_{i}$ and $p_{j}$, since the shortest path can not be shorter than the straight line segment between the two points. Note that $\hat{\delta}_{i, j}^{\text {lower }}$ is not necessarily worst-case optimal.

An upper bound on $\hat{\delta}_{i, j}$ is given by $\delta_{i, j}$. This upper bound is optimal in the worst case, since a path tracing a hole of $S$ can be the shortest path on $\hat{S}$ if $\hat{S}$ has a high mountain where the hole is located on $S$. Note however, that the upper bound only exists if there exists a path on $S$ between $p_{i}$ and $p_{j}$. If this is not the case, $p_{i}$ and $p_{j}$ are located on two distinctively connected components of $S$. In this case, the error of the approximation $d_{i, j}$ is not bounded from above. Hence, only an error bound of infinity can be given. Note that this error bound is optimal, because we can always find a surface $\hat{S}$ where the geodesic distance $\hat{\delta}_{i, j}$ between $p_{i}$ and $p_{j}$ exceeds any given finite length by building high mountains between the regions corresponding to the distinct connected components containing $p_{i}$ and $p_{j}$ on $S$.

Once a lower bound $\hat{\delta}_{i, j}^{\text {lower }}$ and an upper bound $\hat{\delta}_{i, j}^{\text {upper }}$ are known, the relative error $e$ of the approximation of $\hat{\delta}_{i, j}$ by $d_{i, j}$ is computed as $e=\frac{\max \left(\left|d_{i, j}-\hat{\delta}_{i, j}^{\text {lower }}\right|,|| d_{i, j}-\hat{\delta}_{i, j}^{\text {upper }} \mid\right)}{d_{i, j}}$.

\subsection{Description of the Algorithm}

We now describe the algorithm used to estimate geodesic distances on the incomplete surface $S$. Initially, a set $P$ of indices of sample points on $S$ is obtained as discussed in Section 3.3.1. The algorithm proceeds in three steps. First, the fast marching technique is performed to obtain all of the pairwise geodesic distances $\delta_{i, j}, i, j \in P$ on $S$ along with confidence values $\omega_{i, j}, i, j \in P$. Second, we use the geodesics and confidence values to compute the canonical form of $S$. Third, we estimate the geodesic distance between $p_{i}$ and $p_{j}$ by adding $p_{i}$ and $p_{j}$ to the canonical form and by computing the Euclidean distance in embedding space. 
First, to obtain all of the pairwise geodesic distances $\delta_{i, j}, i, j \in P$ on $S$ along with confidence values $\omega_{i, j}, i, j \in P$, the fast marching technique is used. Note that the fast marching technique does not compute exact geodesic distances on $S$, but approximations. However, the geodesic distances computed via the fast marching technique approximate $\delta_{i, j}$ well for surfaces obtained using a laser-range scanner in practice, since the longest edge of $S$ and the widest angle of the triangles in the triangulation of $S$ are small. Furthermore, the triangular mesh is already an approximation of the exact geometry of the object that was scanned. Hence, a good approximation of the geodesics along the triangular approximation of the surface is sufficient. If the triangular mesh is not approximating a smooth surface, the minor theoretical flaw of using geodesic distances computed via the fast marching technique instead of the exact geodesic distances on $S$ can further be overcome by using any known exact algorithm to compute geodesic distances on $S[78,102]$. The main advantage of choosing the fast marching technique is that it can be combined with Voronoi sampling to obtain an elegant and efficient sampling approach [79]. Furthermore, the fast marching technique is easy to implement.

Second, the pairwise geodesic distances $\delta_{i, j}, i, j \in P$ on $S$ along with confidence values $\omega_{i, j}$ are used to perform least-squares MDS and to obtain a canonical form. Since the aim is not to reduce the dimensionality of the data, we choose $k=3$ and $k=4$ as dimensions of the embedding space in our experiments. Instead of starting with a random point set, we initialize the canonical form to the canonical form computed using classical MDS. This reduces the risk of getting stuck in a local minimum when performing the iterations required for least-squares MDS, since classical MDS cannot get stuck in local extrema.

Third, we estimate the geodesic distance between any pair $p_{i}$ and $p_{j}$ of vertices on $S$. Note that $i$ and $j$ do not have to be elements of $P$. To estimate the geodesic distance, we first compute the geodesic distance $\delta_{i, j}$ between $p_{i}$ and $p_{j}$ via the fast marching technique and analyze the resulting geodesic path. If the path does not trace a hole of $S$, a valid geodesic path was found. We report the result along with an error bound of zero, since the exact geodesic path was found. Otherwise, the path traces a hole of $S$. If $i \notin P(j \notin P$ respectively $), p_{i}$ ( $p_{j}$ respectively) is projected to 
the canonical form as discussed in Section 3.2. To project $p_{i}$ to the canonical form, all of the geodesic distances $\delta_{i, r}, r \in P$ and weights $\omega_{i, r}, r \in P$ are computed via the fast marching technique and an optimization problem with $k$ variables is solved using a quasi-Newton method. Once the embedded points $X_{i}$ and $X_{j}$ are known, we use the Euclidean distance $d_{i, j}(X)$ in embedding space to approximate the geodesic distance between $p_{i}$ and $p_{j}$ on $S$. The approximation error of $d_{i, j}(X)$ is bounded by $\max \left(\left|\hat{d}_{i, j}-d_{i, j}(X)\right|,\left|\delta_{i, j}-d_{i, j}(X)\right|\right)$. Recall that this error bound is finite if and only if there exists a path from $p_{i}$ to $p_{j}$ on $S$.

An overview of the algorithm as chart is given in Figure 3.2.

\begin{tabular}{l}
$\begin{array}{l}\text { Preprocessing performed once } \\
\text { per surface } S \text {. }\end{array}$ \\
Obtain $n_{P}$ samples. \\
$\begin{array}{l}\text { Compute pairwise geodesic dis- } \\
\text { tances between the samples us- } \\
\text { ing FMM on } S \text {. }\end{array}$ \\
$\begin{array}{l}\text { Compute } \delta_{i, j} \text { and } \omega_{i, j} \text { based on } \\
\text { the pairwise geodesic distances } \\
\text { and paths of the samples. }\end{array}$ \\
$\begin{array}{l}\text { Use } \delta_{i, j} \text { and } \omega_{i, j} \text { to compute an } \\
\text { embedding of the } n_{P} \text { samples } \\
\text { via least-squares MDS. }\end{array}$ \\
\hline
\end{tabular}

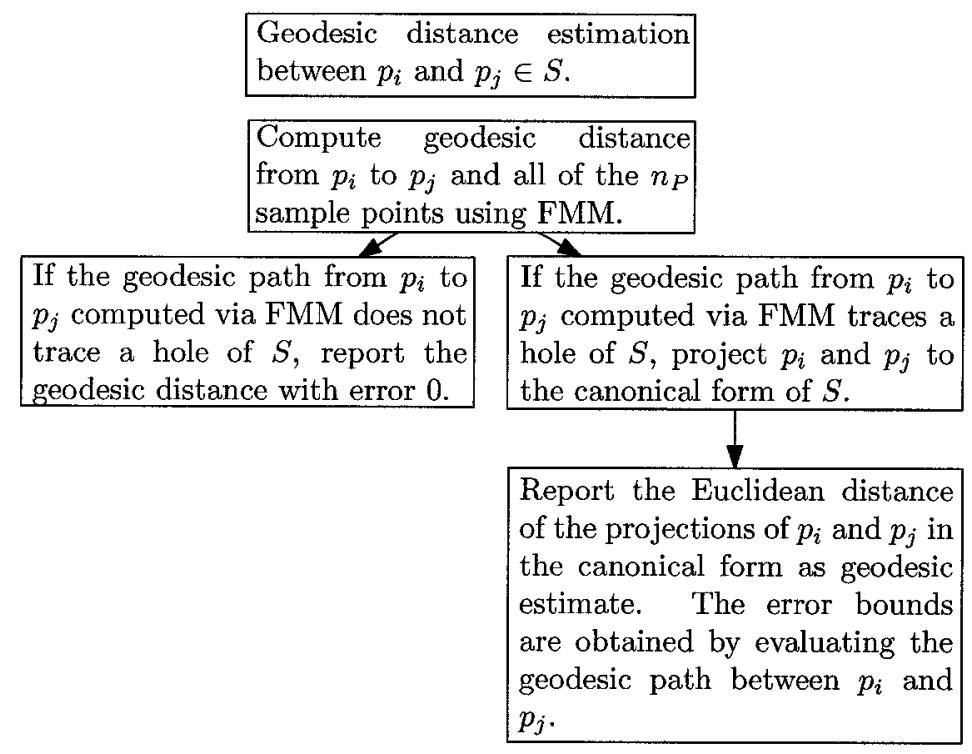

Figure 3.2: Chart describing the overview of the proposed algorithm. The left side of the chart describes the preprocessing performed once per surface. The right side of the chart describes the steps taken to compute the geodesic distance between two points on the surface after preprocessing.

\subsection{Analysis of the Algorithm}

We first analyze the running time of the first two steps of the algorithm. Note that computing the canonical form once per surface $S$ can be viewed as a preprocessing step. 
The first step computes the $\left(\begin{array}{c}n_{P} \\ 2\end{array}\right)$ geodesic paths on $S$ via the fast marching technique, which takes $O\left(n_{P} n \log n\right)$ time. The second step computes the canonical form given the weights and dissimilarities, which takes $O\left(n_{P}^{2} t\right)$ time for least-squares MDS, where $t$ is the number of iterations required for convergence. Hence, the preprocessing step of the algorithm takes $O\left(n_{P}\left(n \log n+n_{P} t\right)\right)$ time.

Computing all geodesic distances and weights needed to project $p_{i}$ and $p_{j}$ to the canonical form in step three of the algorithm takes $O(n \log n)$ time. Furthermore, solving an optimization problem of $k$ variables takes $O\left(k n_{P} t\right)$ time, where $t$ is the number of iterations required for convergence. Since $k$ is a constant, step three of the algorithm takes $O\left(n \log n+n_{P} t\right)$ time.

From above, it follows that after $O\left(n_{P}\left(n \log n+n_{P} t\right)\right)$ preprocessing time, this algorithm reports in $O\left(n \log n+n_{P} t\right)$ time an approximation of the geodesic distance between $p_{i}$ and $p_{j}$ on $S$ along with a worst-case optimal upper bound. Note that both the preprocessing time and the approximation time are dominated by the time needed to compute $\delta_{i, j}$ using the fast marching algorithm. If we can find a faster way to perform this computation, the algorithm's running time is expected to decrease significantly.

\subsection{Experimental Results}

We evaluated the quality of approximation of the approach using three real-life data sets. One aim of the tests performed is to evaluate the influence of the sampling approaches on the approximation. The first data set is the model of a bag that was reconstructed from multiple images. The model consists of 7091 vertices, 520 of which were used to compute a canonical form. We used 511 test vertices that were not used to compute the canonical form to evaluate the quality of the geodesic approximations. The model is shown in Figures 3.3 (a) and (c). Vertices used to compute the canonical form are shown in red and vertices used to evaluate the quality of the geodesic approximation are shown in green. Figure 3.3 (a) shows the samples obtained using curvature-based sampling and Figure 3.3 (c) shows the samples obtained using Voronoi sampling. The samples in Figure 3.3 (c) are more evenly distributed over the model 
surface than the samples in Figure Figure 3.3 (a). Holes in the model are visible in blue. Figures 3.3 (b) and (d) show the canonical forms obtained via least-squares MDS with $\mathbb{R}^{3}$ as embedding space.

The results of the quality of the approximations of geodesic distances between the green sample points for $\mathbb{R}^{3}$ and $\mathbb{R}^{4}$ as embedding space are shown in the first two rows of Table 3.1. All of the error bounds in Table 3.1 are given as relative error bounds. The first row discusses the results when least-squares MDS is performed with $\mathbb{R}^{3}$ as embedding space. Note that when Voronoi sampling is used, $83.87 \%$ of the geodesic distance approximations have a relative error under 0.25 . This means that for $83.87 \%$ of the geodesic distance approximations, the approximation $d_{i, j}$ is bounded by $\frac{3}{4} \hat{\delta}_{i, j} \leq d_{i, j} \leq \frac{5}{4} \hat{\delta}_{i, j}$. Furthermore, $99.78 \%$ of the geodesic distance approximations are bounded by $\frac{1}{2} \hat{\delta}_{i, j} \leq d_{i, j} \leq \frac{3}{2} \hat{\delta}_{i, j}$ and all of the geodesic distance approximations are bounded by $\frac{1}{4} \hat{\delta}_{i, j} \leq d_{i, j} \leq \frac{7}{4} \hat{\delta}_{i, j}$. In this example, curvature-based sampling yields slightly better results. The second row discusses the results when least-squares MDS is performed with $\mathbb{R}^{4}$ as embedding space. Allowing a higher-dimensional embedding space yields higher accuracy until the dimension of the embedding space is sufficient. We can see that the quality of the result only changed insignificantly. Hence, we can conclude that $\mathbb{R}^{3}$ is a suitable embedding space for the bag.

We further estimated all the distances from a fixed source point for the bag model. The canonical form used for this experiment is obtained using the Voronoi sampling strategy and $\mathbb{R}^{3}$ as embedding space. The source point is shown as red dot in Figure 3.4. Figure 3.5 (a) shows a color display of the distance estimates from the source point. The color scale shows small distances as blue color and far distances as red color. Figure 3.5 (b) shows a color display of the corresponding error bounds. Detail views of the hole boundary of the same color-displayed model are shown in Figure 3.5 (c) and (d), respectively. The color scale shows small error bounds as blue color and large error bounds as red color. We can see that the distance estimate proceeds smoothly around the hole of the bag. However, the error bounds get larger close to the hole of the bag, since some shape information is not available.

The two other data sets are human models from the Civilian American and European Surface Anthropometry Resource (CAESAR) data base that were acquired 

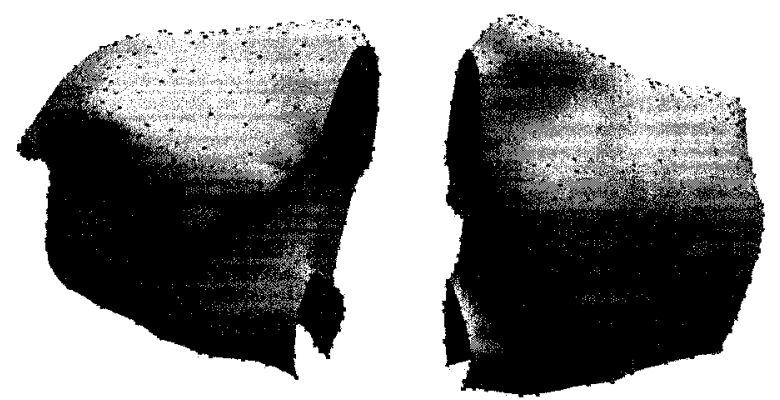

(a)

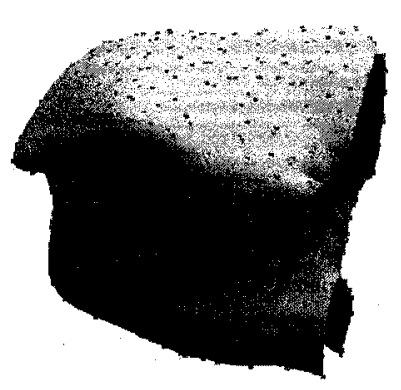

(c)

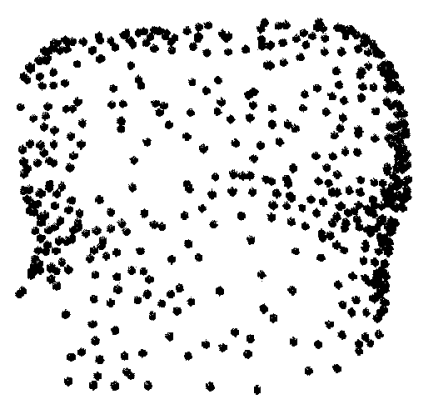

(b)

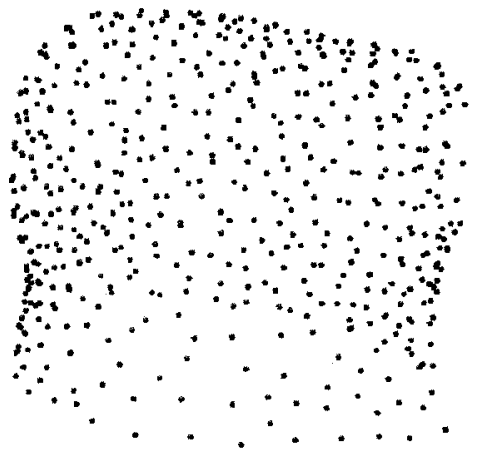

(d)

Figure 3.3: (a), (c) Model of a bag obtained by image-based reconstruction. The points used to compute the canonical form are shown in red and the test vertices are shown in green. (b), (d) Canonical form of the bag obtained via least-squares MDS. (a), (b) Obtained by curvature-based sampling, (c), (d) Obtained by Voronoi sampling.

using a 3D range scans. The first of the human models consists of 209660 vertices, 3707 of which were used to compute a canonical form. We used 1218 test vertices that were not used to compute the canonical form to evaluate the quality of the geodesic approximations. The model is shown in Figures 3.6 (a) and (c). Vertices used to compute the canonical form are shown in red and vertices used to evaluate the quality of the geodesic approximation are shown in green. Holes in the model are visible in blue. Figures 3.6 (b) and (d) show the canonical form with $\mathbb{R}^{3}$ as embedding space obtained for the model. Figure 3.7 shows a detailed view of holes on the arm and upper body of the model.

The results of the quality of the approximations of geodesic distances between the green sample points for $\mathbb{R}^{3}$ and $\mathbb{R}^{4}$ as embedding space are shown in the third 


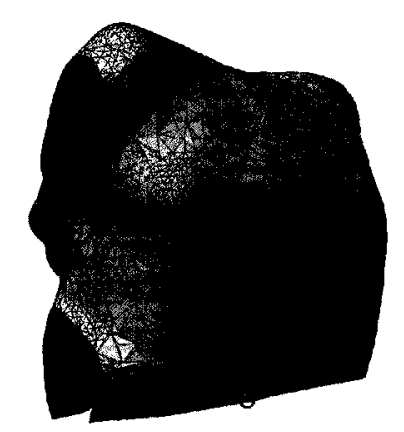

Figure 3.4: Bag model with red dot as source point for SSSP computation.

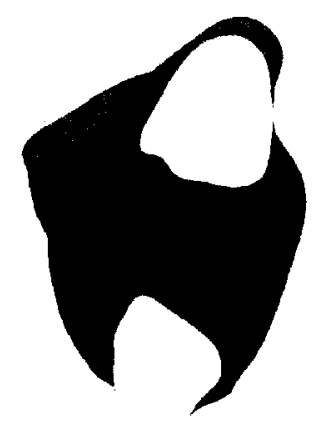

(a)

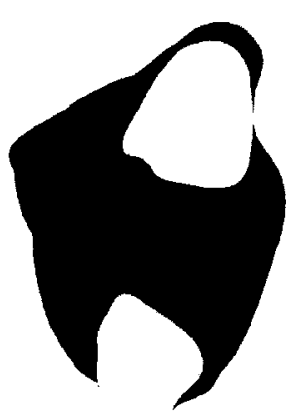

(b)

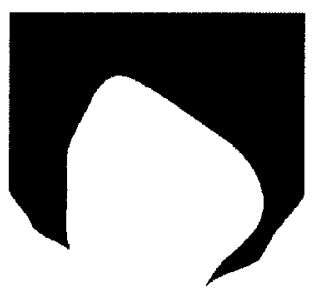

(c)

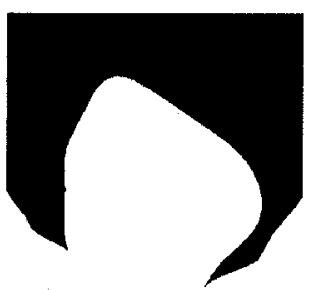

(d)

Figure 3.5: (a) Color display of the geodesic distance estimates from one source point. Blue means small distance and red means large distance. (b) Color display of the error bounds of the distances shown in (a). Blue means small error bound and red means large error bound. (c) Detail view of (a). (d) Detail view of (b).

and fourth rows of Table 3.1. The third row discusses the results when least-squares MDS is performed with $\mathbb{R}^{3}$ as embedding space. Note that although less than $2 \%$ of the original 209660 vertices of the model were used as samples to compute the canonical form, $99.50 \%$ of the bounded geodesic distance approximations have a relative error under 0.75 when Voronoi sampling is used. We can see that the result varies significantly depending on which sampling approach is used to compute the sample set. Computing the canonical form using Voronoi sampling yields higher accuracy. The fourth row discusses the results when least-squares MDS is performed with $\mathbb{R}^{4}$ as embedding space. Again, we can see that $\mathbb{R}^{3}$ is a suitable embedding space for the model, since the quality of the result only changes insignificantly by allowing an embedding in higher dimensions. 

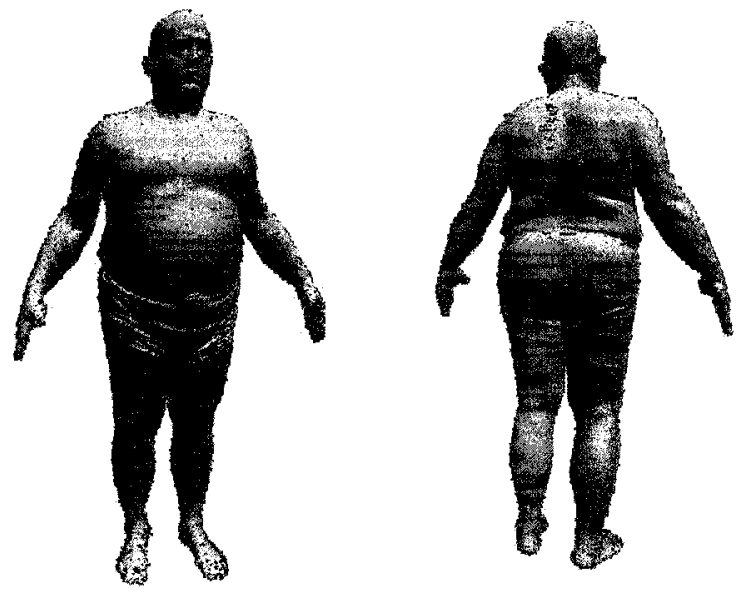

(a)

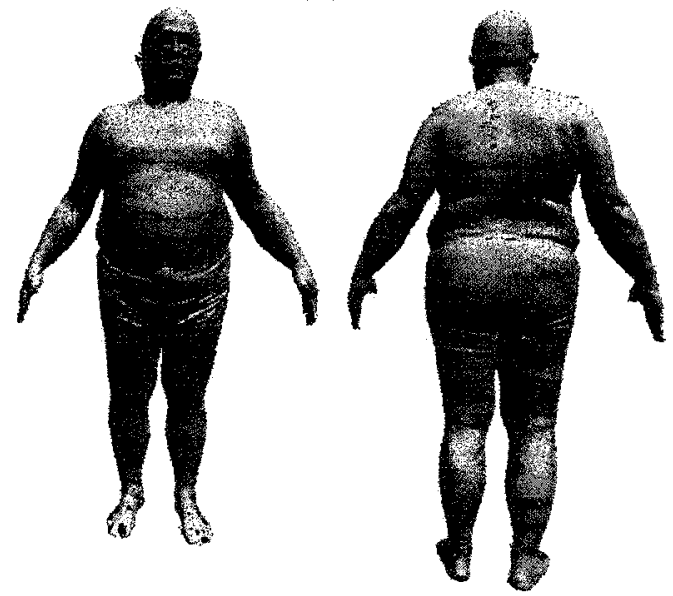

(c)

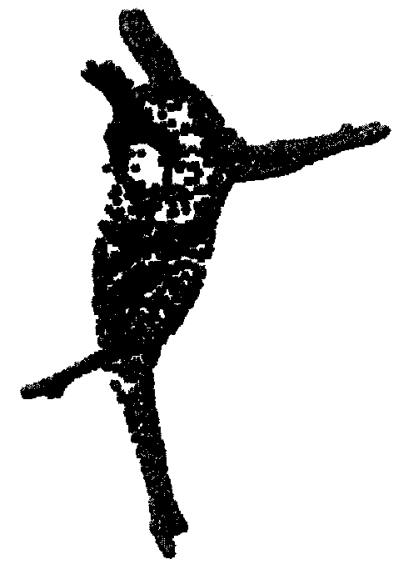

(b)

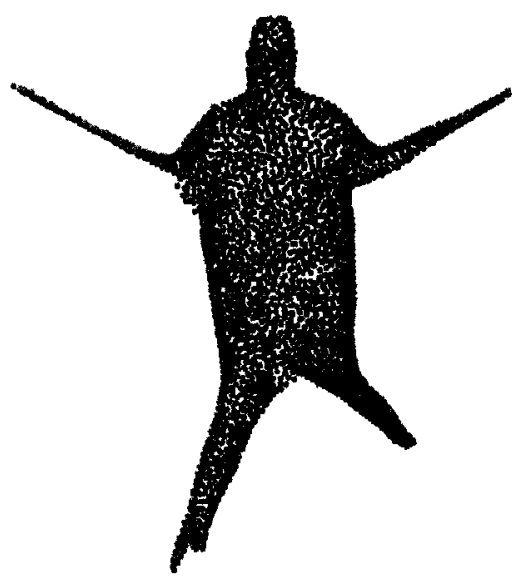

(d)

Figure 3.6: Model csr0001a. (a), (c) The points used to compute the canonical form are shown in red and the test vertices are shown in green. (b), (d) Canonical form of csr0001a obtained via least-squares MDS. (a), (b) Obtained by curvature-based sampling, (c), (d) Obtained by Voronoi sampling. 


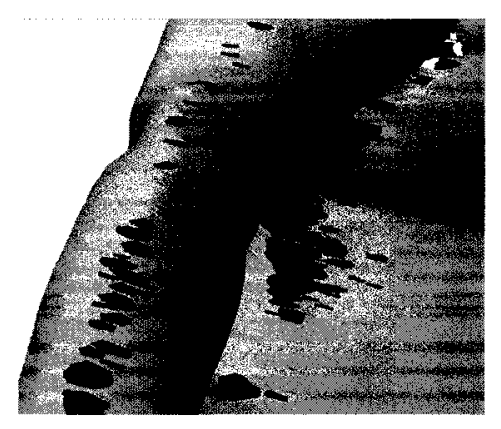

Figure 3.7: Model csr0001a. Detail views of holes on arm and upper body.

The second of the human models consists of 160190 vertices, 3707 of which were used to compute a canonical form. We used 1218 test vertices that were not used to compute the canonical form to evaluate the quality of the geodesic approximations. The model is shown in Figures 3.8 (a) and (c). Vertices used to compute the canonical form are shown in red and vertices used to evaluate the quality of the geodesic approximation are shown in green. Holes in the model are visible in blue. Figures 3.8 (b) and (d) show the canonical form with $\mathbb{R}^{3}$ as embedding space obtained for the model.

The results of the quality of the approximations of geodesic distances between the green sample points for $\mathbb{R}^{3}$ and $\mathbb{R}^{4}$ as embedding space are shown in the fifth and sixth rows of Table 3.1. The fifth row discusses the results when least-squares MDS is performed with $\mathbb{R}^{3}$ as embedding space. The sixth row discusses the results when least-squares MDS is performed with $\mathbb{R}^{4}$ as embedding space. Again, we can see that a relatively small sample set yields satisfying results with $\mathbb{R}^{3}$ as embedding space. Furthermore, the result varies significantly depending on which sampling approach is used to compute the sample set. As before, computing the canonical form using Voronoi sampling yields higher accuracy. 


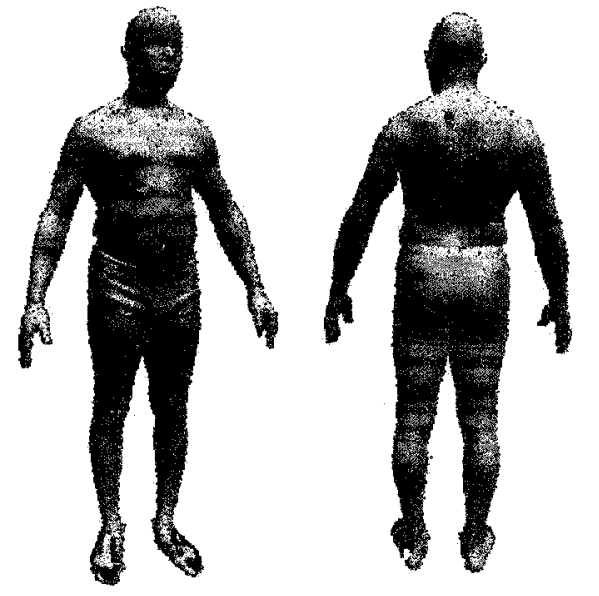

(a)

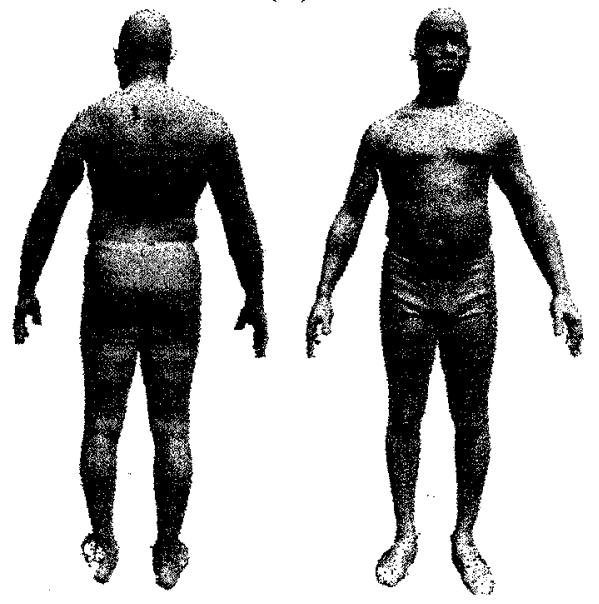

(c)

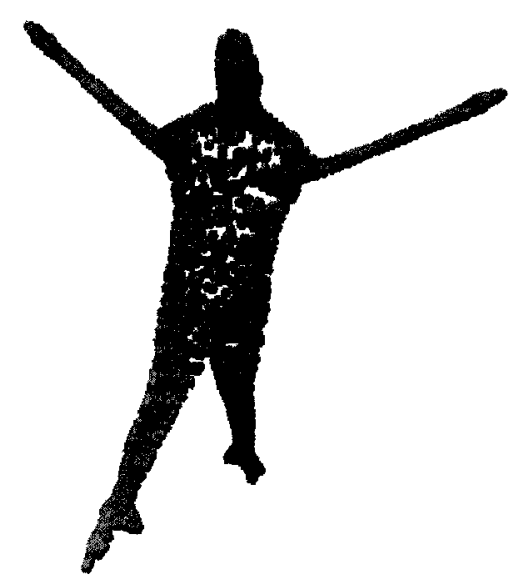

(b)

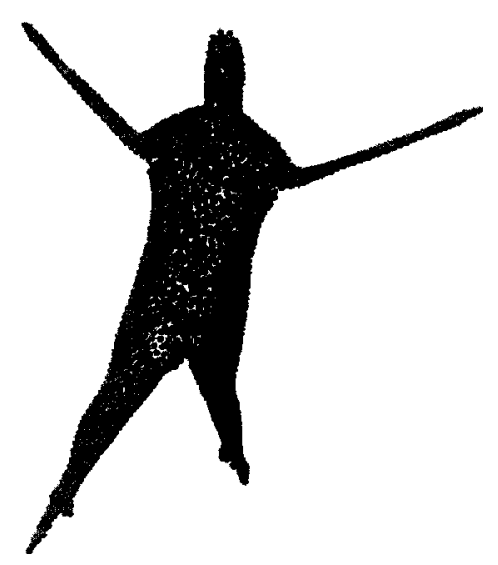

(d)

Figure 3.8: Model csr0106a. (a), (c) The points used to compute the canonical form are shown in red and the test vertices are shown in green. (b), (d) Canonical form of csr0106a obtained via least-squares MDS. (a), (b) Obtained by curvature-based sampling, (c), (d) Obtained by Voronoi sampling. 


\begin{tabular}{|c|c|c|c|c|}
\hline $\begin{array}{c}\text { Object Embedding, Sampling Approach/ } \\
\% \text { of errors under } \\
\text { error bound }\end{array}$ & 0.25 & 0.5 & 0.75 & 1 \\
\hline \multicolumn{5}{|l|}{ bag } \\
\hline MDS $\mathbb{R}^{3}$, Curvature-Based & 89.70 & 99.89 & 100.00 & 100.00 \\
\hline MDS $\mathbb{R}^{3}$, Voronoi & 83.87 & 99.78 & 100.00 & 100.00 \\
\hline MDS $\mathbb{R}^{4}$, Curvature-Based & 89.70 & 99.89 & 100.00 & 100.00 \\
\hline $\operatorname{MDS} \mathbb{R}^{4}$, Voronoi & 83.87 & 99.78 & 100.00 & 100.00 \\
\hline \multicolumn{5}{|l|}{ csr0001a } \\
\hline MDS $\mathbb{R}^{3}$, Curvature-Based & 58.91 & 84.24 & 98.58 & 99.59 \\
\hline MDS $\mathbb{R}^{3}$, Voronoi & 75.15 & 93.07 & 99.50 & 99.90 \\
\hline MDS $\mathbb{R}^{4}$, Curvature-Based & 59.28 & 85.09 & 98.85 & 99.75 \\
\hline MDS $\mathbb{R}^{4}$, Voronoi & 75.14 & 93.12 & 99.48 & 99.95 \\
\hline \multicolumn{5}{|l|}{ csr0106a } \\
\hline MDS $\mathbb{R}^{3}$, Curvature-Based & 57.61 & 77.51 & 96.14 & 98.51 \\
\hline MDS $\mathbb{R}^{3}$, Voronoi & 69.64 & 87.12 & 98.43 & 99.75 \\
\hline MDS $\mathbb{R}^{4}$, Curvature-Based & 58.24 & 78.72 & 96.99 & 99.22 \\
\hline MDS $\mathbb{R}^{4}$, Voronoi & 70.47 & 87.67 & 98.65 & 99.92 \\
\hline
\end{tabular}

Table 3.1: Quality of approximation. The table shows the percentage of estimated errors with finite error bounds within relative error bounds of $0.25,0.5,0.75$, and 1 , respectively. A relative error bound of 0.5 implies that the true geodesic distance is at least half and at most 1.5 times the estimated geodesic distance.

The above experiments show that $\mathbb{R}^{3}$ is a sufficient embedding space for the models we consider and that Voronoi sampling yields better results. It is to be expected that Voronoi sampling outperforms curvature-based sampling, since Voronoi sampling computes uniformly distributed samples in the geodesic sense while curvature-based sampling creates artificial large holes in the data in areas of low curvature. Since MDS is sensitive with respect to large holes, the canonical form does not represent the intrinsic geometry well when curvature-based sampling is used. Based on this conclusion, in all of the following experiments, Voronoi sampling is employed and $\mathbb{R}^{3}$ is chosen as embedding space.

The accuracy of the approximation was evaluated using a synthetic data set. The complete data set of an artist-created human body consisting of 20002 vertices shown in Figure 3.9 was modified to simulate the holes present in the models of the 
CAESAR data base [85] as shown in Figure 3.9. We found 362 testing samples on the incomplete model using Voronoi sampling. The following distances between each pair of sample points $p_{i}$ and $p_{j}$ with the property that the geodesic path between $p_{i}$ and $p_{j}$ computed via the fast marching technique traces at least 20 boundary edges of $S$ are considered: the true geodesic distance $\hat{\delta}_{i, j}$ computed via the fast marching technique on the complete surface, the upper bound $\hat{\delta}_{i, j}^{u p p e r}$ computed via the fast marching technique on the incomplete surface, and the estimate $d_{i, j}$ along with a relative error bound $e_{i, j}$ computed as proposed in this chapter using 4000 samples to compute the canonical form. We used these distances to find the true relative errors of both $\hat{\delta}_{i, j}^{u p p e r}$ and $d_{i, j}$. A histogram of the error bounds $e_{i, j}$ and relative errors of $\hat{\delta}_{i, j}^{u p p e r}$ and $d_{i, j}$ is shown in Figure 3.10. Figure 3.10 (a) shows the number of occurrences of relative errors in the bins shown at the $x$-axis. The error bound $e_{i, j}$ overestimates the error of $d_{i, j}$ in most cases. Figure 3.10 (b) shows the percentage of distances where the relative error of $d_{i, j}$ is smaller than or equal to the relative error of $\hat{\delta}_{i, j}^{u p p e r}$. We can see that $\hat{\delta}_{i, j}^{u p p e r}$ is more accurate than $d_{i, j}$ for small relative error bounds. For larger relative error bounds, $d_{i, j}$ is more accurate than $\hat{\delta}_{i, j}^{u p p e r}$.

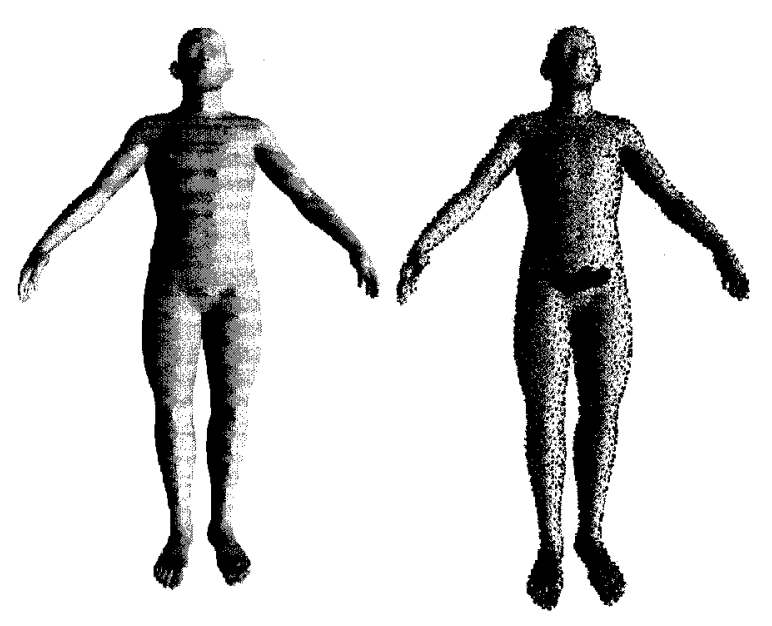

Figure 3.9: Model of a human model. Left: Complete model. Right: Modified model. The points used to compute the canonical form are shown in red and the test vertices are shown in green.

In a further experiment to evaluate the accuracy of the approximation, a complete data set was modified to contain holes in order to evaluate the difference between the 


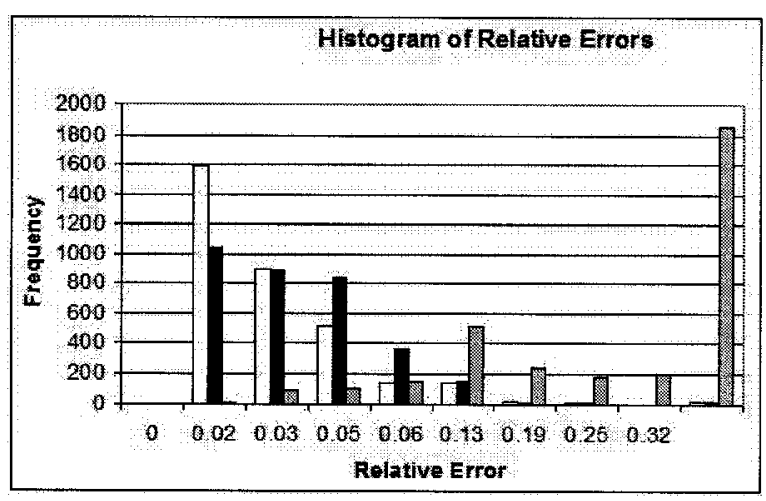

(a)

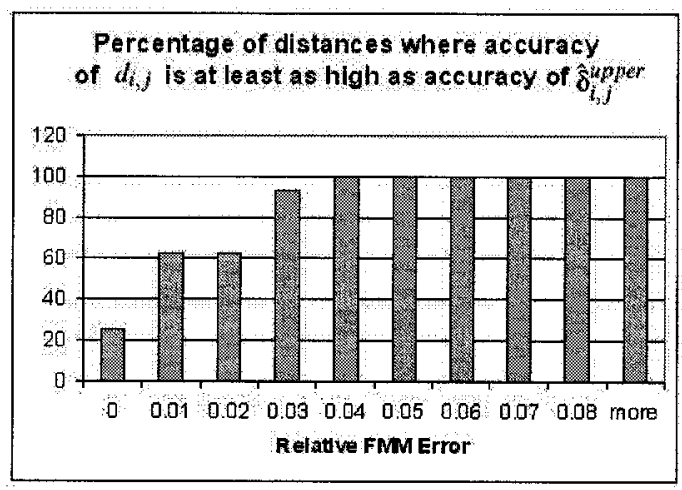

(b)

Figure 3.10: (a) Histogram shows the number of times that relative error bound in a certain bin occurs. The occurrence of $\hat{\delta}_{i, j}^{u p p e r}$ is shown in white, the occurrence of $d_{i, j}$ is shown in black, and the occurrence of $e_{i, j}$ is shown in grey. (b) The columns show the percentage of distances where the relative error of $d_{i, j}$ is smaller than or equal to the relative error of $\hat{\delta}_{i, j}^{u p p e r}$.

worst-case optimal upper bound and the true error. The distance between the two red points on the complete model of a human head shown in Figure 3.11 is computed using the fast marching technique. After, the model is modified to contain holes resembling the holes present in subjects of the CAESAR data base. The holes are shown in blue in Figure 3.11. We use 201 samples to compute the distance between the corresponding points on the incomplete model using the approach presented in this chapter with $\mathbb{R}^{3}$ as embedding space. Although the computed relative error bound for the distance estimation is $8.25 \%$, the true relative error made in this case is only $2.51 \%$. The relative error of the fast marching distance on the incomplete model is 10.97\%. This shows that for subjects of the CAESAR data base, in non-pathological cases a sample size of $1 \%$ of the original model size and a low-dimensional embedding space are sufficient to obtain errors that are significantly smaller than the computed error bound. 


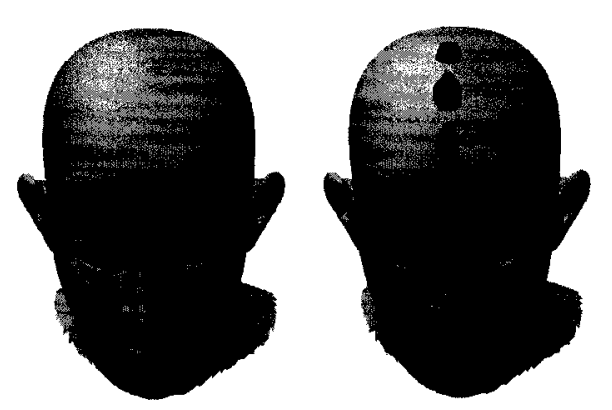

Figure 3.11: Left: Complete model of a human head used to compute the geodesic distance between the two red points via the fast marching technique. Right: Incomplete model of the same head used to compute the geodesic estimate between the two red points using the approach presented in this chapter.

\subsection{Conclusion}

In this chapter we presented an algorithm to compute approximate geodesic distances on triangular manifolds with partially missing data. The geodesic distance approximations have worst case optimal error bounds. The geodesic approximation algorithm is shown to be feasible for large data sets with hundreds of thousands of vertices. The experimental results illustrate that the relative error bounds are below 0.25 for most approximations even for surfaces with significant amounts of missing data.

Computing approximate geodesic distances allows us to examine the intrinsic geometry of a triangular manifold. We use this in Chapter 5 .

In this chapter, we derived a new method to add an object to the embedding computed via least-squares MDS. This method is generally applicable to problems involving least-squares MDS.

Finally, we give ideas for future work.

- An interesting open question is to find an easily computable worst-case optimal lower bound of $\hat{\delta}_{i, j}$.

- Another open question is how to choose the confidence values $\omega_{i, j}$ to optimize the quality of the estimate. 


\section{Chapter 4}

\section{Graph Embedding in Linear Space}

An attempt at visualizing the Fourth Dimension: Take a point, stretch it into a line, curl it into a circle, twist it into a sphere, and punch through the sphere.

Albert Einstein (1879-1955)

A problem arising when embedding the surface in a low-dimensional space via MDS in Chapter 3 is that computing the embedding via least-squares MDS takes quadratic space. This limits the practical application of MDS to small data sets for pragmatic reasons. In Chapter 3, we bypass this problem by computing an embedding of an automatically computed sample set of $S$.

This chapter considers computing the distance preserving graph embedding problem using linear space. The distance preserving graph embedding problem is to embed the vertices of a given weighted graph onto points in $d$-dimensional Euclidean space for a constant $d$ such that for each edge the distance between their corresponding endpoints is as close to the weight of the edge as possible.

This chapter presents a linear-space algorithm to solve the embedding problem. A key idea is to partition a set of $n$ objects into $O(\sqrt{n})$ disjoint subsets (clusters) of size $O(\sqrt{n})$ each, such that the minimum inter cluster distance is maximized among all possible such partitions. Experimental results on applying the algorithm to canonical representations of manifold meshes are included. 


\subsection{Introduction}

Suppose a set of $n$ objects is given and for each pair of objects $(i, j)$ their dissimilarity denoted by $\delta_{i, j}$ can be computed in constant time. Using the dissimilarity information, we want to map the objects onto points in a low dimensional space while preserving the dissimilarities as the distances between the corresponding points.

Converting distance information into coordinate information is helpful for human perception because we can see how close two objects are. Many applications require the embedding of a set of high dimensional objects while preserving dissimilarities for data analysis and visualization. Examples include analysis of psychological models, stock markets, computational chemistry problems, and medical images. Because of its practical importance, this topic has been widely studied under the name of dimension reduction [11]. The application targeted in this thesis is to examine the intrinsic geometry of a triangular manifold $S$ to solve the correspondence problem. We therefore apply the newly developed embedding algorithm to the computation of canonical representations of manifold meshes.

Recall that Multi-Dimensional Scaling (MDS) discussed in Section 2.3 is a generic name for a family of algorithms for dimension reduction. Although MDS is powerful, it has a serious drawback for practical use due to its high space complexity. The input to MDS is an $n \times n$ matrix specifying the pairwise dissimilarities (or distances). In this chapter, we present a method for dimension reduction that avoids this high space complexity if the dissimilarity information is given by a function that can be evaluated in constant time. If the dissimilarities are specified by a matrix, we cannot avoid quadratic space.

A key idea of our linear-space implementation is to use clustering in the first step. That is, given a set of $n$ objects with a function evaluating the dissimilarities for pairs of objects, we partition the set into $O(\sqrt{n})$ disjoint subsets called clusters. More precisely, using a positive constant $m$, we partition the set into $k=O\left(\frac{n}{m}\right)$ subsets $C_{1}, C_{2}, \ldots, C_{k}$ such that each cluster contains between $m$ and $\mathrm{cm}$ objects, where $c \geq 2$ is a constant, except for possibly one cluster having at most $m$ elements. When we set $m=O(\sqrt{n})$ then the number, $k$, of clusters and the largest cluster size is bounded by 
$O(\sqrt{n})$. Now, each cluster has size $O(\sqrt{n})$, and thus performing MDS with a distance matrix for each cluster separately requires only $O(n)$ working space.

The quality of the output depends heavily on the clustering. In many applications, dissimilarities between similar objects have more importance than those between totally dissimilar objects. We propose a simple algorithm for finding a size-constrained clustering stated above that satisfies the following property: We prove that our clustering achieves the largest inter-cluster distance, which means maximizes the smallest distance between objects from different clusters.

\subsection{Related Work}

This section reviews work related to dimension reduction and clustering. We first focus on dimension reduction algorithms.

Problems where we wish to embed dissimilarities as points in a low-dimensional space often arise in many different settings including data visualization [41] and computer graphics [22]. The goal is to find meaningful low-dimensional subspaces that are hidden in the high-dimensional observations. Low-dimensional subspaces are especially useful to visualize the high-dimensional data in a meaningful way.

A popular dimension reduction technique is MDS. For a review of MDS, refer to Section 2.3. Two other popular algorithms for dimension reduction are locally linear embedding [87] and isomap [103]. The locally linear embedding is similar to our algorithm in that the points are clustered and clusters are later recombined. However, the clustering used in the locally linear embedding is a heuristic and cannot be proven to satisfy the property that the minimum distance between objects in two different clusters is maximized. We show that the clustering presented in this chapter has this desirable property. Furthermore, the recombination step of the locally linear embedding requires quadratic working space. Isomap embedding uses classical MDS as a subroutine and therefore uses quadratic working space. The advantage of using an isomap embedding is that the algorithm can be proven to converge to the globally optimal embedding. Another class of dimension reduction algorithms aims to analytically bound the worst embedding error [24,25]. 
Clustering is important in the area of pattern recognition, where the goal is to find clusters in data represented as points in $d$ dimensions. We give a short summary of common clustering methods as described by Duda et al. [32]. Assume that we can measure the similarity between any pair of samples. We aim to find the best partition of the data set into $c$ clusters. To derive algorithms for this, we need to define how we evaluate a partition of the samples into clusters. This summary reviews two commonly used methods to evaluate a partitioning of the samples into clusters. The first method aims to minimize the sum-of-squared errors between each sample and the mean of the cluster containing the sample. More formally, the aim is to minimize $J=\sum_{i=1}^{c} \sum_{\vec{x} \in D_{i}}\left\|\vec{x}-\vec{m}_{i}\right\|^{2}$, where $D_{i}$ is the $i$-th cluster and $\vec{m}_{i}$ is the mean of the $i$-th cluster. Clusterings that minimize $J$ are also denoted minimum variance partitions. The error function $J$ measures the error made when representing the entire sample set using only the means $\vec{m}_{i}$ of the $c$ clusters. A second commonly used method evaluates the partition of the data set into $c$ clusters based on the scatter matrix $S_{T}=S_{W}+S_{B}$, where $S_{W}=\sum_{i=1}^{c} \sum_{\vec{x} \in D_{i}}\left(\vec{x}-\vec{m}_{i}\right)\left(\vec{x}-\vec{m}_{i}\right)^{T}$ with $\vec{m}_{i}$ as mean of the $i$-th cluster is the within-cluster scatter matrix, $S_{B}=\sum_{i=1}^{c} n_{i}\left(\vec{m}_{i}-\vec{m}\right)\left(\vec{m}_{i}-\vec{m}\right)^{T}$ with $\vec{m}$ as mean of all samples and $n_{i}$ as the number of samples in cluster $D_{i}$ is the between-cluster scatter matrix, and $S_{T}$ is the total scatter matrix. There exist several ways to define cost functions based on the scatter matrix. The trace criterion aims to minimize $\operatorname{tr}\left(S_{W}\right)$. While minimizing $\operatorname{tr}\left(S_{W}\right), \operatorname{tr}\left(S_{B}\right)$ is maximized. The determinant criterion is a scale invariant criterion that aims to minimize $\left|S_{W}\right|$. Further criteria that are invariant under nonsingular linear transformations of the data can be defined using the eigenvalues of the matrix $S_{W}^{-1} S_{B}$. Note that invariant criteria are more likely to possess multiple minima and therefore harder to optimize. Various other ways exist to define criteria to measure the quality of a partition. There is no universally best clustering criterion, but the criterion depends on the given samples and the underlying sample space.

Once we defined a way to evaluate a partition of the samples into clusters, the clustering problem is defined as optimization problem. Most of the resulting optimization problems have local minima and therefore yield different solutions for different initial configurations. Starting from an initial configuration, we can solve the optimization 
problem using gradient-descent or quasi-Newton methods that aim to follow the steepest descent of the gradient of the cost function used to evaluate a partition. Duda et al. [32] outline a way to solve the optimization problem using an iterative approach where random samples are tentatively moved to another cluster and left there if the cost function used to evaluate a partition decreases by this change. The iteration is repeated until no changes occurred in the past $n$ iterative steps.

Another way to solve the problem is a hierarchical approach, where the solution to the $c$-clustering problem is derived from the solution to the $(c-1)$-clustering problem. For example, we can take the total mean of all samples as the mean of the solution to the 1-clustering problem and iteratively add the farthest sample from all means to obtain a $c$-clustering from a $(c-1)$-clustering. This general approach is called hierarchical clustering and hierarchical clusters are desired if clusters can contain subclusters. The concept of hierarchical clustering is simple and commonly used. There are two main hierarchical approaches. Divisive methods start with one cluster and iteratively split the cluster. One example of a divisive clustering method was given above. Agglomerative methods build a hierarchy by starting with $n$ singleton clusters and by iteratively merging the two closest clusters according to a suitable distance measure.

We review four commonly used distance definitions to measure distances between two clusters. First, the distance between two clusters $D_{i}$ and $D_{j}$ can be defined as the closest distance between vertices $\vec{x}_{k} \in D_{i}$ and $\vec{x}_{l} \in D_{j}$. This approach is known as nearest-neighbor algorithm and for $c=1$, the minimum spanning tree of the sample points is obtained. Second, the distance between two clusters $D_{i}$ and $D_{j}$ can be defined as the farthest distance between vertices $\vec{x}_{k} \in D_{i}$ and $\vec{x}_{l} \in D_{j}$. This approach is known as farthest-neighbor algorithm. The farthest-neighbor algorithm prevents elongated clusters. Both the nearest-neighbor method and the farthest-neighbor method suffer from the fact that small changes in the sample positions can significantly change the clustering result. Therefore, two further methods are discussed that can be seen as compromises of the first two methods. In these methods, sensitivity to outliers is avoided. For the third method, the distance between two clusters $D_{i}$ and $D_{j}$ is defined as the average distance between vertices $\vec{x}_{k} \in D_{i}$ and $\vec{x}_{l} \in D_{j}$. For the fourth method, 
the distance between two clusters $D_{i}$ and $D_{j}$ is defined as the distance between the means of the two clusters.

Hierarchical clustering is also called multigrid clustering in some applications. Various hierarchical clustering methods exist. For instance, Kushnir et al. [64] introduce a clustering method that finds a multigrid clustering based on algebraic multigrid solvers commonly used in fluid dynamics.

So far, we assumed that we know the number $c$ of clusters we aim to find. However, in many cases, we do not know how many classes the given samples belong to. When extremizing a criterion function using hierarchical clustering, we can find the most natural number of clusters by running the hierarchical clustering algorithm with $c=n$. We choose the estimated number $\hat{c}$ of natural clusters, such that the extremum of the criterion function improves significantly when allowing $c+1$ clusters instead of $c$ clusters for $c \leq \hat{c}$ and such that the extremum of the criterion function improves by an insignificant amount when allowing $\hat{c}+1$ clusters instead of $\hat{c}$. A more formal way is to define the null hypothesis that there are $c$ clusters present and to test the hypothesis based on the sampling distribution for $c+1$ clusters.

We can view the clustering problem from a graph theoretical point of view. Our newly developed algorithm is similar to the second graph theoretical algorithm reviewed below. The first approach truncates the similarity matrix $S$, which contains the similarities between samples $\vec{x}_{i}$ and $\vec{x}_{j}$ at position $s_{i, j}$, such that

$$
s_{i, j}=\left\{\begin{array}{l}
1 \text { if similarity } \vec{x}_{i}, \vec{x}_{j}>r \\
0 \text { otherwise }
\end{array}\right.
$$

where $r$ is a threshold. We can view the dual matrix of $S$ as the matrix representation of a graph $G$. The graph $G$ is called the similarity graph of the samples. The clustering induced by the connected components of $G$ equals the clustering obtained by the nearest-neighbor algorithm if we stop when the closest distance between clusters exceeds $r$. Furthermore, the clustering induced by the complete subgraphs of $G$ equals the clustering obtained with the farthest-neighbor algorithm if we stop when the closest distance between clusters exceeds $r$. The second approach starts with the minimum spanning tree of the samples. Removing the longest edges of the minimum 
spanning tree yields two clusters. We can repeat this hierarchically and after removing the $c-1$ longest edges from the minimum spanning tree, we are left with $c$ clusters. Our approach extends this technique.

Another graph theoretical approach to clustering employs dominant sets on graphs, where each edge has a weight associated to it. The weights can be viewed as similarities between the two endpoints of the edge. The goal is to cluster vertices that are connected by edges of high weight. A dominant set $S$ is a subset of the vertices $V$, such that edge weights associated with edges between two vertices in $S$ have higher weight than edges between one vertex in $S$ and one vertex in $V \backslash S$. That is, the overall similarity among internal vertices in $S$ is higher than the similarity between internal and external vertices. When unweighted graphs are considered, dominant sets are equivalent to strictly maximal cliques. Dominant sets were applied to clustering tasks in computer vision, such as shape recognition [82] and image segmentation [81].

\subsection{Clustering}

In this section, we consider the problem of partitioning a set of objects into clusters according to the values of the pairwise dissimilarities. We show that it is possible to find a size-constrained partitioning with largest inter-cluster distance using linear space.

Let $S$ be a set of $n$ objects: $S=\{1, \ldots, n\}$. We assume that we are given a function which computes the dissimilarity between any pair $(i, j)$ of objects as $\delta_{i, j}$ with $\delta_{i, i}=0$ and $\delta_{i, j}=\delta_{j, i}>0$ for $i \neq j$. A partition $\mathcal{P}$ of a set $S$ into $k$ disjoint clusters $C_{1}, \ldots, C_{k}$ is called a $k$-partition of $S$. A $k$-partition $\mathcal{P}$ is characterized by two distances, inner-cluster distance $D_{i n n}(\mathcal{P})$ and inter-cluster distance $D_{\text {int }}(\mathcal{P})$, which are defined by

$$
D_{i n n}(\mathcal{P})=\max _{C_{i} \in \mathcal{P}} \max _{p, q \in C_{i}} \delta_{p, q}
$$

and

$$
D_{\text {int }}(\mathcal{P})=\min _{C_{i} \neq C_{j} \in \mathcal{P}} \min _{p \in C_{i}, q \in C_{j}} \delta_{p, q}
$$

When we define a complete graph $G(S)$ with edge weights being dissimilarities, edges 
are classified as inner-cluster edges connecting vertices of the same cluster and intercluster edges connecting vertices of different clusters. The inner-cluster distance is the largest weight of inner-cluster edges and the inter-cluster distance is the smallest weight of inter-cluster edges.

A $k$-partition is called farthest (most compact, respectively) if it is a $k$-partition with largest inter-cluster distance (smallest inner-cluster distance, respectively) among all $k$-partitions. Given a set $S$ of $n$ objects, we want to find a $k$-partition of $S$ which is farthest and most compact. It is generally hard to achieve the two goals simultaneously. In fact, the problem of finding a most compact $k$-partition, even in the special case where the dissimilarities come from a metric space, is NP-hard [38]. However, in important special cases we can find such a $k$-partition rather easily. That is the case of a well-separated partition.

\subsubsection{Well-Separated Partitions}

A $k$-partition $\mathcal{P}$ of a set $S$ is called well-separated if $D_{\text {inn }}(\mathcal{P})<D_{\text {int }}(\mathcal{P})$. If there is a well-separated $k$-partition of a given set then we can find a $k$-partition that is farthest and most compact. Moreover the partition is unique.

Lemma 4.3.1. Let $S$ be a set of $n$ objects with a dissimilarity defined for every pair of objects. If $S$ has a well-separated $k$-partition, then it is unique provided that no two pairs of objects have the same dissimilarity. The unique well-separated $k$-partition is farthest and most compact.

Proof. Uniqueness of a well-separated $k$-partition follows from the assumption that no two pairs of objects have the same dissimilarity. A $k$-partition classifies edges of a complete graph $G(S)$ defined by dissimilarities into inner-cluster edges and intercluster edges. Then, the inner-cluster distance $D_{i n n}(\mathcal{P})$ is achieved by the largest inner-cluster edge and the inter-cluster distance $D_{\text {int }}(\mathcal{P})$ by the smallest inter-cluster edge. Since $\mathcal{P}$ is well-separated, the weight of every inner-cluster edge is smaller than the weight of any inter-cluster edge. So, if we change any edge from an inner-cluster to an inter-cluster edge or vice versa then we violate the inequality $D_{\text {inn }}(\mathcal{P})<D_{\text {int }}(\mathcal{P})$. Thus, uniqueness follows. 
Let $\mathcal{P}$ be the unique well-separated $k$-partition of $S$. Then, it is most compact since reducing the inner-cluster distance requires splitting some clusters, which increases the number of clusters. It is also farthest. To prove this by contradiction, suppose there is a $k$-partition (which is not necessarily well separated) with inter-cluster distance $t>D_{\text {int }}(\mathcal{P})$. This means that all the edges which were inner-cluster edges remain unchanged and those edges with weights greater than $D_{i n n}(\mathcal{P})$ and less than $t$ have become inner-cluster edges. Hence, the number of connected components is at most $k-1$. This is a contradiction.

Consider the case where $S$ admits a well-separated $k$-partition. By Lemma 4.3.1, if we sort all the dissimilarities in increasing order then the inter-cluster distance must appear right next to the inner-cluster distance in this order. Hence, it suffices to find some dissimilarity $t^{*}$ such that there is a well-separated $k$-partition with the inner-cluster distance being $t^{*}$. If we define a graph $G_{t}(S)$ as the subgraph of $G(S)$ with the edges of weight at most $t$ then the connected components of $G_{t}(S)$ define a well-separated partition of $S$ with inner-cluster distance $t$. If we can find a dissimilarity $t$ such that the graph $G_{t}(S)$ consists of $k$ connected components, then it is a solution. The following is an algorithm for counting the number of connected components in a graph $G_{t}(S)$ using linear working space. In the algorithm, we first scan every pair and if its weight is at most $t$ then we merge the two clusters containing those objects into one cluster. At this moment we report the number of remaining clusters. After that, we again scan every pair and report NO if we find any pair with dissimilarity greater than $t$ such that both of them belong to the same cluster, and report YES if no such pair is found. Formally, this method is described in Algorithm 1.

If $S$ has a well-separated $k$-partition, the algorithm returns $k$ and YES for dissimilarity $\delta_{i j}$ for some pair $(i, j)$. A naive algorithm is to check all the dissimilarities. Since there are $O\left(n^{2}\right)$ different dissimilarities, $O\left(n^{2}\right)$ iterations of the algorithm are enough. It takes $O\left(n^{4}\right)$ time in total but the space required is $O(n)$.

An idea for an efficient implementation is binary search on the sorted list of dissimilarities. Generally speaking, the larger the $t$ value becomes the fewer subsets we have by the algorithm above. If the output are $k$ and YES for some $t^{*}$, then the resulting partition is the unique well-separated $k$-partition. If there is such a value $t^{*}$, 


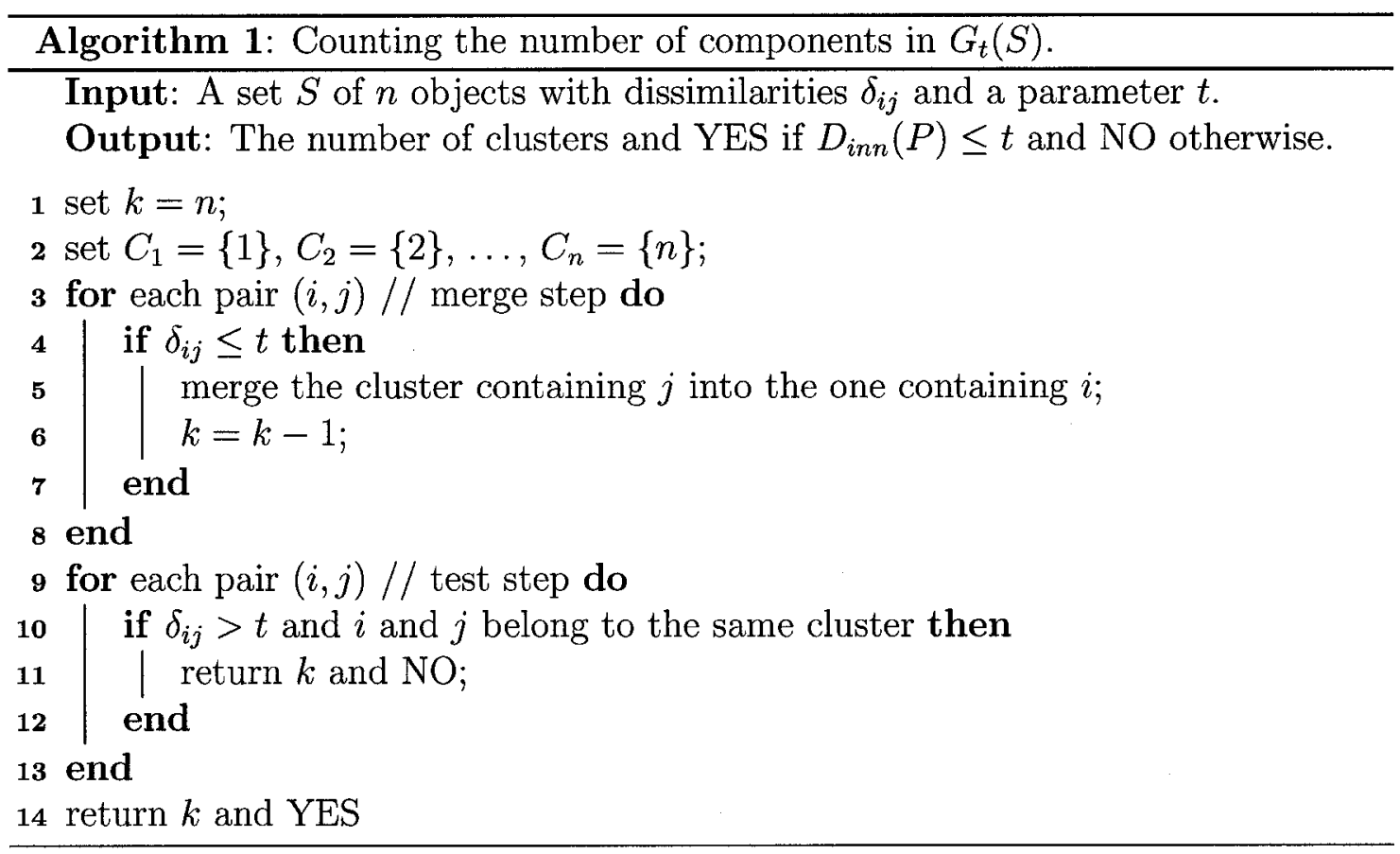

any other value of $t$ with $t>t^{*}$ generates fewer clusters. On the other hand, if we have more than $k$ clusters for some $t$, then we have $t<t^{*}$. There can be many $t$ values that generate exactly $k$ clusters, but $t^{*}$ is the largest value among them. Thus, if the output is NO and the number of clusters is at most $k$ for some $t$, we can conclude that $t<t^{*}$. Based on this observation, we can implement binary search.

Linear-space algorithm for well-separated partition: One serious problem with the method sketched above is that we cannot store a sorted list of dissimilarities due to the linear space constraint. We implement the binary search in two stages. In the beginning, our search interval contains a superlinear number of distances. Hence, we compute an approximate median instead of the exact median. As the binary search proceeds, our interval gets shorter and shorter. Once the number of distances falling into the search interval is at most $c n$ for some positive constant $c$, we can find an exact median. A more detailed description follows:

We start our binary search from the initial interval $\left[1,\left(\begin{array}{c}n \\ 2\end{array}\right)\right]$ which corresponds to a distance interval determined by the smallest and largest distances. We maintain 
an index interval $[$ low, high $]$ corresponding to the distance interval $\left[\delta_{l o w}, \delta_{h i g h}\right]$, where $\delta_{i}$ denotes the $i$-th smallest distance. Imagine dividing the interval [low, high] into four equal parts. An approximate median is any element contained in the 2nd or 3rd quarters. Thus, half of the elements in [low, high] are approximate medians. Equivalently, a random element is an approximate median with probability $1 / 2$.

To find an approximate median, we pick a random integer $k$ with $1 \leq k \leq$ $h i g h-l o w+1$. We can evaluate the dissimilarity function in the order in which the dissimilarities are encountered when scanning the (unknown) distance matrix row by row to simulate scanning the distance matrix. We refer to this process, which uses only $O(1)$ space, as scanning the matrix. We scan the matrix row by row and pick the $k$-th element $X$ with $\delta_{\text {low }} \leq X \leq \delta_{\text {high }}$ that we encounter. Given $X$, we scan the matrix and count the number of values between $\delta_{\text {low }}$ and $X$, and also count the number of values between $X$ and $\delta_{\text {high }}$. In this way, we find out if $X$ is an approximate median. If it is not, then we repeat the above. We know that the expected number of trials is 2. Assume that $X$ is an approximate median. While doing the above, we also find the index $m$ such that $X=\delta_{m}$. Now we test if $X$ is equal/larger/smaller than $D_{i n n}$. If they are equal, we are done. Assume $X$ is less than $D_{i n n}$. Then, we set the right boundary high of our current interval to $m$. If $X$ is larger than $D_{i n n}$, then we set the left boundary low to $m$.

In this way, we spend $O\left(n^{2}\right)$ time for one binary search step. Since the expected number of these steps is $O(\log n)$, the overall expected time bound is $O\left(n^{2} \log n\right)$. Once the current interval contains at most $c n$ distances, we can apply an exact median finding algorithm although we have to scan the matrix in $O\left(n^{2}\right)$ time.

Theorem 4.3.2. Given $n$ objects, a function evaluating the dissimilarity between any pair of objects in $O(1)$ time, and an integer $k<n$, we can decide whether or not there is a well-separated $k$-partition in $O\left(n^{2} \log n\right)$ expected time and $O(n)$ working space using approximate median finding. Moreover, if there is such a partition, we find it in the same time and space.

If we are allowed to use $O(n \log n)$ space, then we can further improve the running time using another randomization technique as follows. For ease of notation, assume 
that the dissimilarities are numbers $1,2,3, \ldots, n^{2}$. Define the interval $I_{i}=[(i-$ $\varepsilon / 2) n,(i+\varepsilon / 2) n]$ for $i=1,2, \ldots, n$. We would like to obtain one element from each of these intervals. We choose a random sample consisting of $c n \log n$ elements.

The probability that this sample leaves one of the intervals $I_{i}$ empty is at most the probability that we did not choose any element of $I_{1}$

+ the probability that we did not choose any element of $I_{2}+\cdots$

+ the probability that we did not choose any element of $I_{n}$.

All probabilities above are equal, so let us look at the probability that we did not choose any element of $I_{1}$. The probability that a random element is not in $I_{1}$ is equal to $1-(\varepsilon n) / n^{2}=1-\varepsilon / n \leq e^{-\varepsilon / n}$. So the probability that none of the $c n \log n$ random elements is in $I_{1}$ is at most $\left(e^{-\varepsilon / n}\right)^{c n \log n}$, which is $n^{-c^{\prime}}$ for some constant $c^{\prime}$. Thus the total probability that our random sample is not $\operatorname{good}$ is at most $n \cdot n^{-c^{\prime}}$, which is at most $1 / 2$. In other words, the probability that the random sample is good is at least $1 / 2$. This means that we expect to do the random sampling at most twice. Hence, the expected running time of the algorithm is $O\left(n^{2}\right)$.

\subsubsection{Farthest and Most Compact $k$-Partition}

The problem of finding a most compact $k$-partition with the smallest inner-cluster distance is difficult since even in the special case that the dissimilarities come from a metric space, finding the most compact $k$-partition for $k \geq 3$ is NP-hard [38, 43]. However, we can find a farthest $k$-partition rather easily.

Let $e_{1}, e_{2}, \ldots, e_{n-1}$ be the edges of a minimum spanning tree $M S T(S)$ for a complete graph $G(S)$ defined for a set $S$ of $n$ objects, and assume that

$$
\left|e_{1}\right| \leq\left|e_{2}\right| \leq \cdots \leq\left|e_{n-1}\right|
$$

Let $M S T_{k}(S)$ be the set of components resulting after removing the $k-1$ longest edges $e_{n-1}, \ldots, e_{n-k+1}$ from $M S T(S)$. Then, $M S T_{k}(S)$ has exactly $k$ components, which defines a $k$-partition of $S$. The following lemma has been claimed by Asano et al. [6] and is proven in Kleinberg and Tardos [59, p. 160].

Lemma 4.3.3. Given a set $S$ of $n$ objects with all pairwise dissimilarities defined, 
removing the $k-1$ longest edges of $M S T(S)$ gives an algorithm for finding a farthest $k$-partition in $O\left(n^{2}\right)$ time and linear working space.

\subsubsection{Size-Constrained Farthest Partition}

Recall that our aim is to embed the graph using only $O(n)$ space. In order to use MDS for the embedding, clusters that are farthest are not sufficient. We also need to ensure that the clusters are sufficiently small and that there are not too many of them. Specifically, we need to find $O(\sqrt{n})$ clusters of size $O(\sqrt{n})$ each. For a given $m$ with $1 \leq m<n$, define the farthest partition satisfying the size constraint on $m$ as a clustering $\mathcal{P}$ satisfying three properties: all clusters contain between $m$ and $\mathrm{cm}$ objects with $c \geq 2$, at most one cluster contains at most $m$ objects with $1<m<n$, and $D_{i n t}(\mathcal{P})$ is maximized. The method outlined in Lemma 4.3 .3 does not provide such a partition.

To find the farthest partition satisfying the size constraint on $m$ given a set $S$ of $n$ objects, consider the following algorithm. First, each object $i$ is placed into a separate cluster $C_{i}$ of size one to initialize the algorithm. The algorithm iteratively finds the minimum remaining dissimilarity $\delta_{i j}$. If merging the cluster $C_{l}$ containing object $i$ and cluster $C_{q}$ containing object $j$ does not violate the size constraint, that is, if it does not produce a new cluster of size exceeding $\mathrm{cm}$, the algorithm merges $C_{l}$ and $C_{q}$ into one cluster $C_{l}$. Dissimilarity $\delta_{i j}$ is then removed from consideration. These steps are iterated until all of the dissimilarities are removed from consideration. The formal algorithm is given as Algorithm 2.

Lemma 4.3.4. Given $m$ with $1<m<n$, algorithm Size-constrained-farthestpartition $(m)$ creates a partition such that all clusters contain between $m$ and $\mathrm{cm}$ objects except for at most one cluster that contains at most $m$ objects.

Proof. None of the clusters created by this algorithm has size greater than $\mathrm{cm}$, since otherwise, the clusters would not have been merged by the algorithm. It remains to prove that there exists at most one cluster of size at most $m$. Assume for the sake of a contradiction that there exist two clusters $C_{l}$ and $C_{q}$ of size at most $m$. Since $C_{l}$ and $C_{q}$ have not yet been merged, all $\delta_{i j}$ with $i \in C_{l}$ and $j \in C_{q}$ have not yet been 


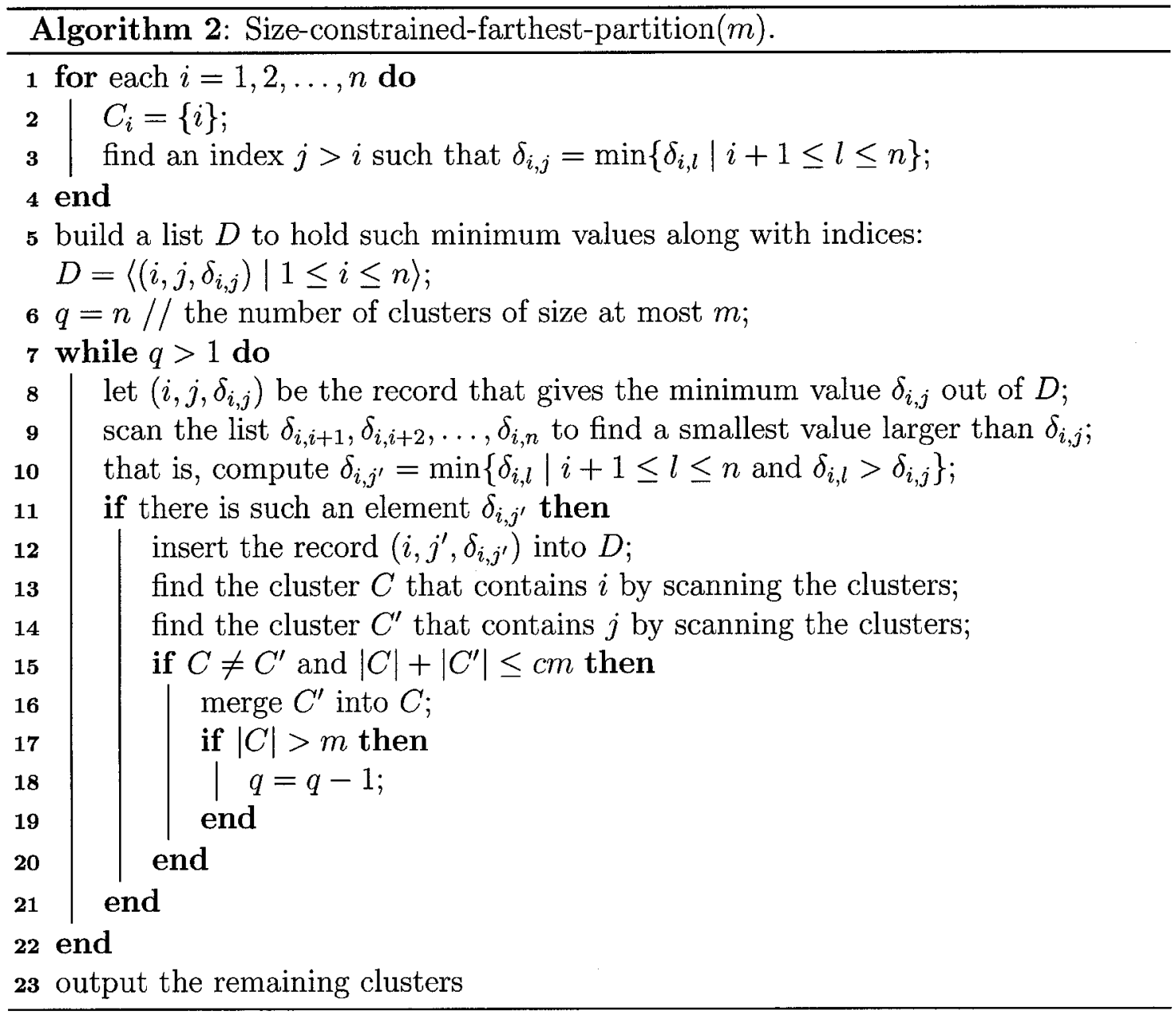


removed from consideration. Hence, the algorithm has not yet reached its stopping criteria, which contradicts the assumption. Hence, the clusters $C_{l}$ and $C_{q}$ are merged before the termination of the algorithm and therefore, there exists at most one cluster that contains at most $m$ vertices.

All clusters contain at most $\mathrm{cm}$ vertices and at most one cluster contains at most $m$ vertices. Hence, according to the pigeon hole principle, the algorithm creates $k$ clusters with $\left\lfloor\frac{n}{c m}\right\rfloor<k \leq\left\lceil\frac{n}{m}\right\rceil+1$.

Lemma 4.3.5. The partition created by algorithm Size-constrained-farthest-partition $(m)$ is farthest among all partitions with the property that all clusters contain at most $\mathrm{cm}$ vertices and at most one cluster contains at most $m$ vertices.

Proof. Assume for the sake of a contradiction that the partition $\mathcal{P}$ created by the algorithm described above is not farthest. Hence, there exists a farther optimal partition $\mathcal{P}_{O P T}$ with the property that all clusters contain at most $\mathrm{cm}$ vertices and at most one cluster contains at most $m$ vertices. The partition $\mathcal{P}$ has $D_{i n t}(\mathcal{P})=\delta_{i j}$ with $i \in D_{l}$ and $j \in D_{q}$, where $D_{l}$ and $D_{q}$ are the first clusters that do not get merged by our algorithm. Since $\mathcal{P}_{O P T}$ is farther, $\delta_{i j}$ is an internal edge of $\mathcal{P}_{O P T}$. Since all clusters of $\mathcal{P}_{O P T}$ contain at most $\mathrm{cm}$ vertices, it is impossible for all vertices of $D_{l}$ and $D_{q}$ to belong to one cluster of $\mathcal{P}_{O P T}$. Hence, without loss of generality, there exists a vertex $a$ in $D_{l}$ that is in a different cluster of $\mathcal{P}_{O P T}$ than $i$. Next, we show that there exists a path $p_{a i}$ between $a$ and $i$ inside $D_{l}$ comprised entirely of edges of length at most $\delta_{i j}$. Initially, $i$ and $a$ were located in two different components $C_{i}$ and $C_{a}$ in the execution of the algorithm described above. Since during its execution, the algorithm merged the component $C_{a}$ containing $a$ and the component $C_{i}$ containing $i$ before considering the edge $\delta_{i j}$, there exists an element $b \in C_{a}$ and an element $c \in C_{i}$ with $\delta_{b c} \leq \delta_{i j}$. This argument can be applied recursively to the pairs of vertices $a, b$ and $i$, $c$, respectively, until the path $p_{a i}$, comprised entirely of edges of length at most $\delta_{i j}$, is found. For an illustration of this approach, please refer to Figure 4.1.

Since in $\mathcal{P}_{O P T}, a$ is in a different cluster than $i$, at least one of the edges on the path $p_{a i}$ is an inter-cluster edge. Hence, the inter-cluster distance $D_{\text {int }}\left(\mathcal{P}_{O P T}\right) \leq$ 


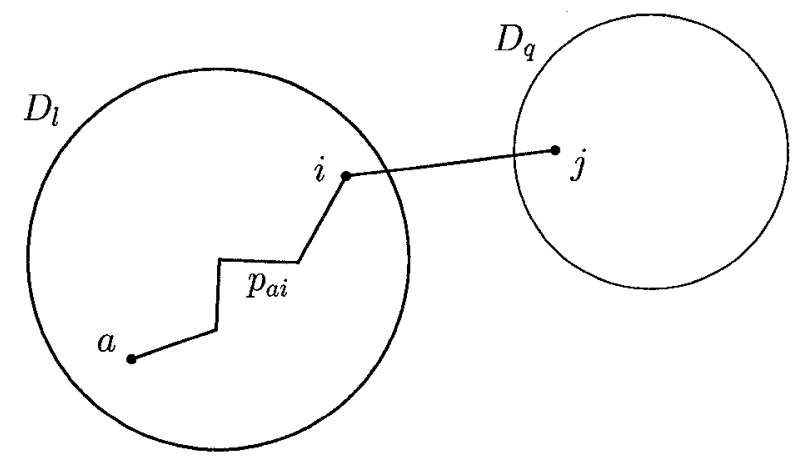

Figure 4.1: Path $p_{a i}$ comprised of short edges between $i$ and a.

$D_{\text {int }}(\mathcal{P})$, which contradicts the initial assumption. This proves that the partition $\mathcal{P}$ is farthest.

To find this partition, we need to find the shortest edge that has not yet been considered iteratively until all the edges were considered. The proposed algorithm is summarized in Algorithm 2. In the algorithm we use a data structures for extracting edges in increasing order of their weights.

To analyze the running time of the algorithm, note that the execution of the for-loop takes $O\left(n^{2}\right)$ time. In the while-loop, there are at most $O\left(n^{2}\right)$ iterations and one execution of the while loop takes $O(n)$ time. Hence, the total running time is $O\left(n^{3}\right)$.

Theorem 4.3.6. One can compute a data structure of linear size that implements the size-constrained farthest partition of $k$ clusters of constraint $m$ in $O\left(n^{3}\right)$ time using $O(n)$ space.

An example of running the clustering algorithm on a set of points in the plane is shown in Figure 4.2. In this example, the dissimilarities between the points are computed as the Euclidean distances between the points. Clusters are shown as connected sets. Cluster centers are shown as black points. 


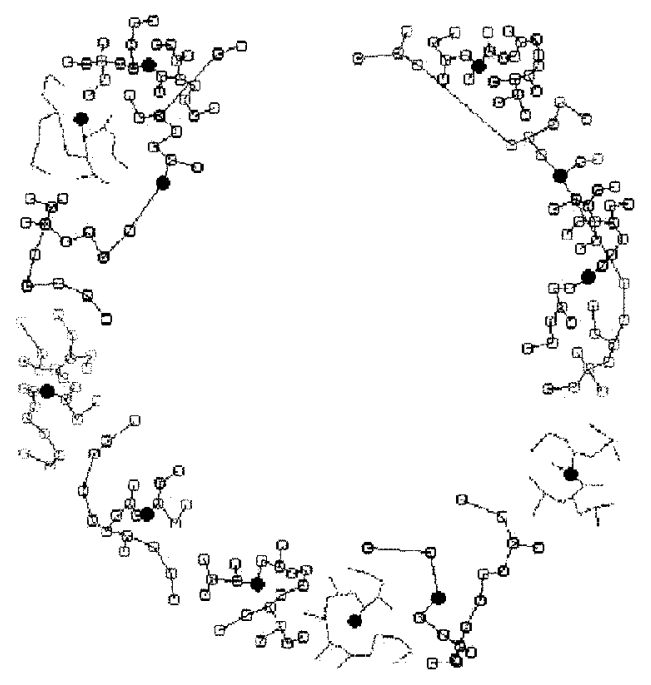

Figure 4.2: Example showing the result of running the clustering algorithm on a random set of 268 points in the plane.

\subsection{Graph Embedding}

A direct way of embedding a weighted graph into a low-dimensional space is to apply least-squares MDS, which needs a full matrix representing dissimilarities between all pairs of objects. This takes $O\left(n^{2}\right)$ space for a set of $n$ objects, which is often a problem for implementation when $n$ is large. To remedy this, we partition the given set into $O(\sqrt{n})$ clusters of size $O(\sqrt{n})$ each by applying Algorithm 2 with $m=\sqrt{n}$. We then embed the clusters using an algorithm similar to the one presented in Section 3.2 to add an additional object to the MDS embedding.

Consider the $k=O(\sqrt{n})$ clusters $C_{1}, C_{2}, \ldots, C_{k}$ with $n_{i}=\left|C_{i}\right|=O(\sqrt{n})$ for each $i$. First we find a center object in each cluster $C_{i}$, denoted by center $\left(C_{i}\right)$, which is defined to be an object in $C_{i}$ such that the largest distance to any other object in that cluster is smallest. We denote the $i$-th cluster by $C_{i}=\left\{p_{1}^{i}, p_{2}^{i}, \ldots, p_{n_{i-1}}^{i}\right\}$. For ease of notation, we exclude the cluster center $p_{i}=p_{n_{i}}^{i}$ from the cluster $C_{i}$ in the following.

Second, we form a set $C_{0}=\left\{p_{1}, p_{2}, \ldots, p_{k}\right\}$ consisting of cluster centers. Since $k=O(\sqrt{n})$, we can apply least-squares MDS to find an embedding that minimizes the sum of squared embedding errors of elements in $C_{0}$ using a distance matrix of size 
$O(n)$. We fix those points as embedded positions $X_{0}=\left[\vec{x}_{1}, \vec{x}_{2}, \ldots, \vec{x}_{k}\right]$.

Third, we embed clusters $C_{1}, C_{2}, \ldots, C_{k}, k=O(\sqrt{n})$, one by one. We do this by minimizing a generalized form of the least-squares MDS energy minimization given in Equation (2.2). The energy that is minimized to embed cluster $C_{i}, i=1,2, \ldots, k$ is

$$
E=\underbrace{\sum_{p_{j} \in C_{i}} \sum_{p_{k} \in C_{i}}\left(\delta_{j, k}-d\left(p_{j}, p_{k}\right)\right)^{2}}_{E_{1}}+\underbrace{\sum_{p_{j} \in C_{i}} \sum_{p_{k} \in C_{0}}\left(\delta_{j, k}-d\left(p_{j}, p_{k}\right)\right)^{2}}_{E_{2}} .
$$

Denote the position vector of the embedding of object $p_{j} \in C_{i}$ in $\mathbb{R}^{d}$ by $\vec{x}_{j}=$ $\left[\begin{array}{llll}x_{j, 1} & x_{j, 2} & \ldots & x_{j, d}\end{array}\right]^{T}$ and denote the point matrix by $X_{i}=\left[\begin{array}{llll}\vec{x}_{i_{1}} & \vec{x}_{i_{2}} & \ldots & \vec{x}_{i_{n_{i}-1}}\end{array}\right]^{T}$.

The first part of the energy function, $E_{1}$, corresponds to the complete least-squares MDS energy if only points in the same cluster $C_{i}$ are considered. The energy $E_{1}$ can therefore be expressed as

$$
E_{1}=\alpha+\beta-\gamma
$$

with

$$
\alpha=\sum_{p_{j} \in C_{i}} \sum_{p_{k} \in C_{i}} \delta_{j, k}^{2}, \beta=\sum_{p_{j} \in C_{i}} \sum_{p_{k} \in C_{i}} d\left(p_{j}, p_{k}\right)^{2}, \text { and } \gamma=2 \sum_{p_{j} \in C_{i}} \sum_{p_{k} \in C_{\boldsymbol{i}}} d\left(p_{j}, p_{k}\right) \delta_{j, k}
$$

and using the Cauchy-Schwartz inequality bounded by

$$
E_{1} \leq \sum_{p_{j} \in C_{i}} \sum_{p_{k} \in C_{i}} \delta_{i, j}^{2}+\operatorname{tr}\left(X_{i}^{T} V_{i} X_{i}\right)-2 \operatorname{tr}\left(X_{i}^{T} B_{i}\left(Z_{i}\right) Z_{i}\right)=\tau^{*}
$$

where $X_{i}$ is a $d \times\left(n_{i}-1\right)$ matrix containing the coordinates of $p_{i}, V_{i}$ is an $\left(n_{i}-1\right) \times\left(n_{i}-1\right)$ matrix with elements

$$
V_{i_{j, k}}=\left\{\begin{array}{l}
n_{i}-1 \text { if } j=k \\
-1 \text { if } j \neq k
\end{array}\right.
$$

$Z_{i}$ is a possible solution for $X_{i}$, and $B_{i}\left(Z_{i}\right)$ is an $\left(n_{i}-1\right) \times\left(n_{i}-1\right)$ matrix with elements

$$
B_{i_{j, k}}=\left\{\begin{array}{l}
-\frac{\delta_{j, k}}{d\left(p_{j}^{i}, p_{k}^{i}\right)} \text { if } j \neq k, d\left(p_{j}^{i}, p_{k}^{i}\right) \neq 0 \\
0 \text { if } j \neq k, d\left(p_{j}^{i}, p_{k}^{i}\right)=0 \\
\sum_{l=1, l \neq j}^{n} B_{l, j} \text { if } j=k
\end{array}\right.
$$


The gradient of $\tau^{*}$ can be found analytically as $\nabla \tau^{*}=2 X_{i}^{T}\left(V_{i}-B_{i}\right)$ (see Section 2.3.2).

The second part of the energy, $E_{2}$, considers the distances between the cluster being embedded and the fixed cluster centers. It is the same energy used in Section 3.2 when adding the points in $C_{i}$ to the fixed embedding of $C_{0}$ one by one. The energy can be rewritten as

$$
E_{2}=\sum_{p_{j} \in C_{i}} \alpha^{*}+\beta^{*}-\gamma^{*}
$$

where $\alpha^{*}=\sum_{p_{k} \in C_{0}} \delta_{j, k}^{2}, \beta^{*}=\sum_{p_{k} \in C_{0}} d\left(p_{j}, p_{k}\right)^{2}$, and $\gamma^{*}=2 \sum_{p_{k} \in C_{0}} \delta_{j, k} d\left(p_{j}, p_{k}\right)$. The gradient of $E_{2}$ can therefore be computed analytically as

$$
\nabla E_{2}=\left[\begin{array}{llll}
\frac{\partial E_{2}}{\partial p_{1}^{i}} & \frac{\partial E_{2}}{\partial p_{2}^{i}} & \cdots & \frac{\partial E_{2}}{\partial p_{n_{i}-1}^{i}}
\end{array}\right]
$$

where $\frac{\partial E_{2}}{\partial p_{k}^{2}}=\sum_{\vec{x}_{j} \in X_{0}} 2\left(\vec{x}_{i_{k}}-\vec{x}_{j}\right)\left(1-\frac{\delta_{j, k}}{d\left(p_{j}, p_{k}^{i}\right)}\right)$, as in Section 3.2.

Hence, we can compute the gradient of the convex function $\tau=\tau^{*}+E_{2}$ bounding $E$ from above with respect to $X_{i}$ analytically as

$$
\nabla \tau=2 X_{i}^{T}(V-B)+\left[\frac{\partial E_{2}}{\partial p_{1}^{i}} \frac{\partial E_{2}}{\partial p_{2}^{i}} \cdots \frac{\partial E_{2}}{\partial p_{n_{i}-1}^{i}}\right]
$$

Since we aim to minimize $E$, we embed each cluster optimally according to the least-squares MDS optimality measure when considering the cluster centers and the objects in the same cluster. We do not take points other than the cluster centers from different clusters into account, since this would require more than linear space. This can be viewed as a trade-off between space and accuracy of the embedding.

The running time of this algorithm, denoted by embedding algorithm in the following, depends on the maximum number of iterations $t$. Embedding the cluster centers takes $O(n d t)$ time and embedding each cluster $C_{i}$ takes $O(n d t)$ time. Hence, the total running time of the embedding algorithm is $O\left(n^{\frac{3}{2}} d t\right)$. Since all of the matrices required for the computation are of size at most $O(\sqrt{n}) \times O(\sqrt{n})$, the algorithm uses linear working space.

Recall that the algorithm to compute a size-constrained farthest partition takes $O\left(n^{3}\right)$ time. This yields the following result:

Theorem 4.4.1. The embedding algorithm embeds a data set $S$ in $\mathbb{R}^{d}$ for any constant embedding dimension $d$ while minimizing the sum of squared embedding errors of 
pairwise dissimilarities between objects in $S$ using $O\left(n^{3}+n^{\frac{3}{2}} d t\right)$ time and $O(n)$ space, where $t$ is the number of iterations required to minimize the least-squares MDS energy.

\subsection{Experimental Results}

We compare our algorithm to two alternative embedding algorithms. The first alternative algorithm is the SMACOF algorithm explained in Section 2.3.2 to compute a least-squares MDS embedding using quadratic space.

The second alternative algorithm is a variant of our algorithm that embeds the cluster centers using SMACOF and simply adds the remaining objects one by one to the embedding as outlined in Section 3.2. We call this algorithm the projection algorithm. The projection algorithm uses a variant of our proposed embedding algorithm as follows. After computing the farthest $k$-partition with $k=\lfloor\sqrt{n}\rfloor$ as presented in Section 4.3, we first embed the cluster centers using the SMACOF algorithm and then add all of the remaining objects to the least-squares MDS embedding one by one as outlined in Section 3.2. This approach takes only linear space, but it does not consider any inner-cluster distances when embedding the objects.

For all embeddings that are computed, we initialize the embedding to the PCO embedding and use the LSBFGS quasi-Newton method for the iterative minimization. For all of our experiments, the constant $c$ for the clustering step is chosen as 2 and the parameter $m$ is chosen as $\lfloor\sqrt{n}\rfloor$. In the following, three applications are considered.

We compare the embedding algorithm to the SMACOF algorithm and the projection algorithm in terms of storage requirement, produced embedding error, running time, and maximum number of iterations $t$ to minimize the energy using LSBFGS. The storage requirement of all algorithms ignores the space to store the data. In case of computing canonical forms, the comparison therefore ignores the space allocated to store the triangular mesh and the data structures required to compute geodesic distances along the mesh. To measure the amount of storage used by the algorithms, we recursively report all the memory that is allocated.

The experiments using three applications show that the time complexity of the input function has a big influence on the running time of the algorithms. It is therefore 
recommended to evaluate the input function as efficiently as possible.

\subsubsection{Embedding Registered High-Energy Gamma Particles}

In a first experiment, we embed the MAGIC gamma telescope data available in the UCI Machine Learning Repository ${ }^{1}$ into $\mathbb{R}^{3}$. The data was generated using a Monte-Carlo method and simulates the registration of high-energy gamma particles in a ground-based atmospheric Cherenkov gamma telescope. The dataset consists of 10-dimensional vectors of real numbers. The distance between two data vectors is computed as Euclidean distance. Note that this implies a low time complexity of the distance function, since the Euclidean distance between 10-dimensional vectors can be computed efficiently. There are a total of 19020 data vectors available in the dataset. The algorithms were tested on five different sizes of datasets. We obtain five datasets of size 1000 to 19020 simply by taking a subset of the original dataset.

Table 4.1 compares the performance of the three algorithms on different sizes of data of the gamma telescope dataset. The table shows the quality of the computed embeddings, the time and space complexities of the algorithms, and the number of iterations required by the algorithms. We do not give the results of the SMACOF algorithm for the two largest datasets, since the algorithm ran out of memory. The time and space requirements are furthermore visualized in Figure 4.3.

The storage required by the projection algorithm and the embedding algorithm are significantly smaller than the storage required by the SMACOF algorithm. This is to be expected, since the SMACOF algorithm requires $O\left(n^{2}\right)$ storage while the other two algorithms require $O(n)$ storage. In this experiment, we see an example where SMACOF is no longer practical due to its quadratic space complexity. For $n=10000$, we can no longer initialize the embedding to the one computed using classical MDS since there is not enough internal memory. This leads to an abnormality in the running time and embedding quality of the SMACOF result for $n=10000$. For $n \geq 15000$, we can no longer run the SMACOF algorithm in internal memory. In order to compute a SMACOF embedding for the datasets of size 15000 or larger, we need to store data in

\footnotetext{
${ }^{1}$ http://archive.ics.uci.edu/ml/
} 
external memory, thereby significantly growing the time complexity of the algorithm. In contrast, the data stored by the embedding algorithm and the projection algorithm still easily fits into internal memory. The projection algorithm requires only about half of the storage required by the embedding algorithm and is the most space efficient algorithm of the three.

The embedding algorithm is the most time efficient algorithm for this dataset. The reason is that the time of evaluating the input dissimilarity function is small compared to the matrix operations used by SMACOF. In general, SMACOF is expected to be the most time efficient algorithm, since its running time is $O\left(t n^{2}\right)$, while the running time of the other two algorithms is $O\left(n^{3}\right)$. The quality of the computed embedding is highest for SMACOF. This does not hold for $n=10000$, since it was no longer possible to initialize the embedding to the classical MDS embedding in this case. We expect SMACOF to be most accurate because SMACOF takes all of the pairwise dissimilarities into account when computing the embedding. The second most accurate embedding is the one produced by the embedding algorithm. This is also expected as the embedding algorithm takes inner-cluster dissimilarities into account while the projection algorithm does not.

\begin{tabular}{|r|r|r|r|r||r|r|r|r||r|r|r|r|}
\hline $\mathbf{n}$ & \multicolumn{4}{|c||}{ Emb. alg. } & \multicolumn{4}{|c||}{ Proj. alg. } & \multicolumn{4}{c|}{ SMACOF } \\
\hline & $t$ & $E_{L S M D S}$ & $S$ & time & $t$ & $E_{L S M D S}$ & $S$ & time & $t$ & $E_{L S M D S}$ & $S$ & time \\
\hline 1000 & 149 & $5.3 \cdot 10^{7}$ & 0.19 & 1 & 43 & $6.5 \cdot 10^{7}$ & 0.10 & 14 & 90 & $2.6 \cdot 10^{7}$ & 20.28 & 17 \\
\hline 5000 & 209 & $1.3 \cdot 10^{9}$ & 0.58 & 18 & 77 & $2.2 \cdot 10^{9}$ & 0.25 & 77 & 117 & $7.0 \cdot 10^{8}$ & 482.73 & 1772 \\
\hline 10000 & 198 & $4.6 \cdot 10^{9}$ & 1.04 & 67 & 48 & $9.8 \cdot 10^{9}$ & 0.42 & 173 & 52 & $7.0 \cdot 10^{10}$ & 1919.02 & 384 \\
\hline 15000 & 219 & $1.8 \cdot 10^{10}$ & 1.48 & 141 & 59 & $3.5 \cdot 10^{11}$ & 0.58 & 301 & \multicolumn{3}{|c|}{ not enough internal memory } \\
\hline 19020 & 182 & $3.6 \cdot 10^{10}$ & 1.82 & 221 & 72 & $1.9 \cdot 10^{12}$ & 0.70 & 422 & \multicolumn{3}{c|}{ not enough internal memory } \\
\hline
\end{tabular}

Table 4.1: Quality of embedding for the gamma telescope dataset. The table shows the maximum number $t$ of iterations required by the LSBFGS quasi-Newton method [73], the embedding error $E_{L S}$ of the computed embedding, the storage use $S$ in $M B$, and the running time in seconds of all three algorithms that were implemented.

\subsubsection{Embedding Grey-Level Images}

The second application is embedding grey-level images of faces into points in the plane. We use the Yale Face Database ${ }^{2}$ showing the faces of 15 individuals. The face of each

\footnotetext{
${ }^{2}$ http://cvc.yale.edu/projects/yalefaces/yalefaces.html
} 


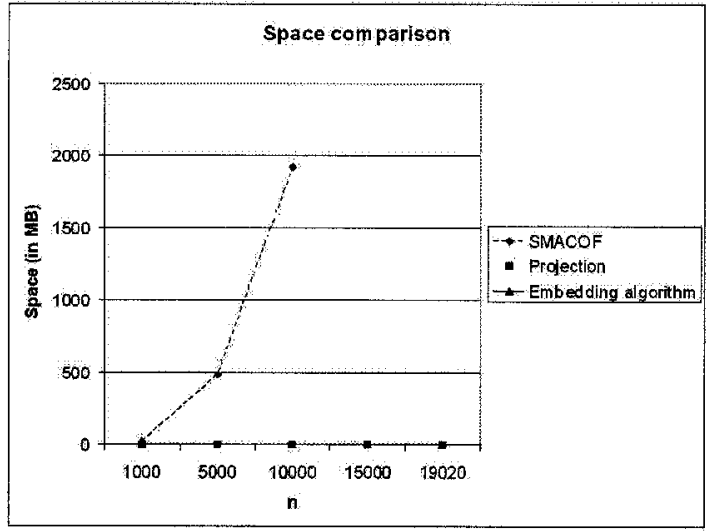

(a)

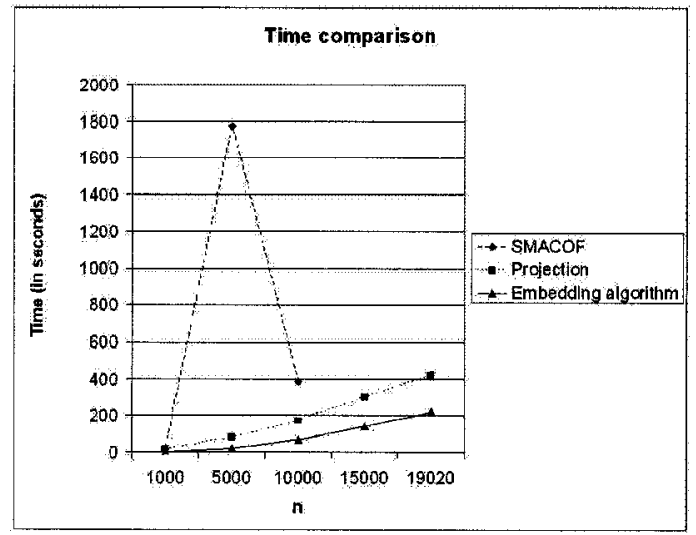

(b)

Figure 4.3: Comparison of time and space used by the embedding algorithm (solid), the projection algorithm (dotted), and the SMACOF algorithm (dashed) for the gamma telescope dataset. The number of objects $n$ is shown along the $x$-axis and the space and time requirements are shown along the y-axis.

individual is shown in 11 different expressions. The eleven facial expressions of one of the subjects in the database are shown in Figure 4.4. An embedding of these faces can be used for classification by subject or expression or for face recognition. Each grey-level image is a vector of dimension 77760 . The distance between two grey-level images is computed as the Euclidean vector-distance. Note that the running time of evaluating this distance function is significantly larger than the running time of evaluating the distance function in the previous experiment because of the higher dimensionality of the data vectors.

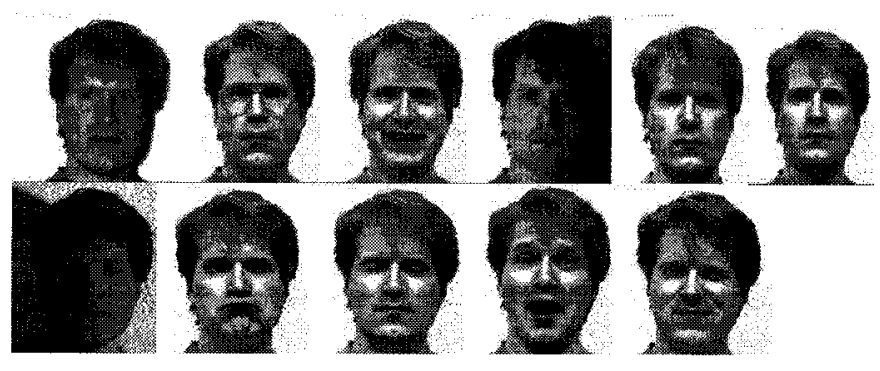

Figure 4.4: Facial expressions in the database.

The results are shown in Table 4.2. We can see that the space used by the 
embedding algorithm and the projection algorithm is significantly lower than the space used by SMACOF. The space used by the projection algorithm is about $70 \%$ of the space used by the embedding algorithm, since no inner-cluster distances need to be stored. The quality of all three embeddings is poor because the faces cannot be represented well in the plane. As expected, the result by SMACOF is best, followed by the result by the embedding algorithm. The SMACOF algorithm is fastest and the embedding algorithm is slowest. This is different than in the previous experiment. The reason is that it takes longer to compute the dissimilarity between two grey-level images than to compute the dissimilarities between two gamma particles.

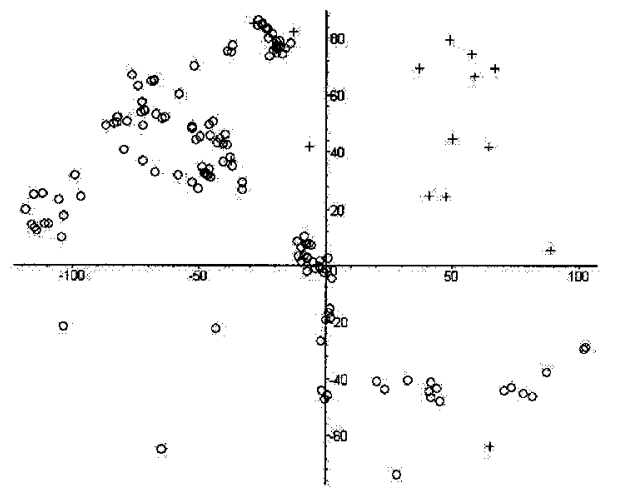

(a)

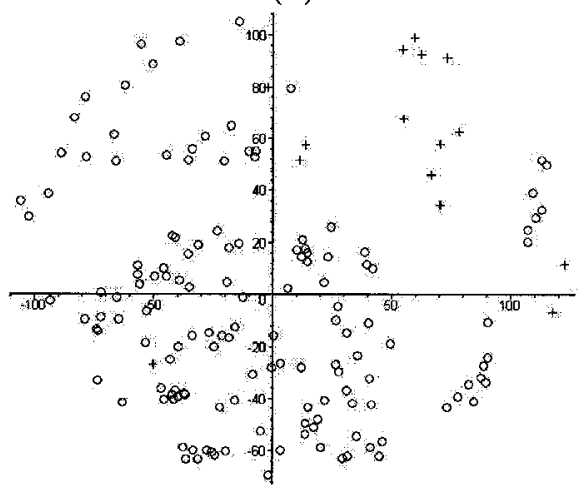

(c)

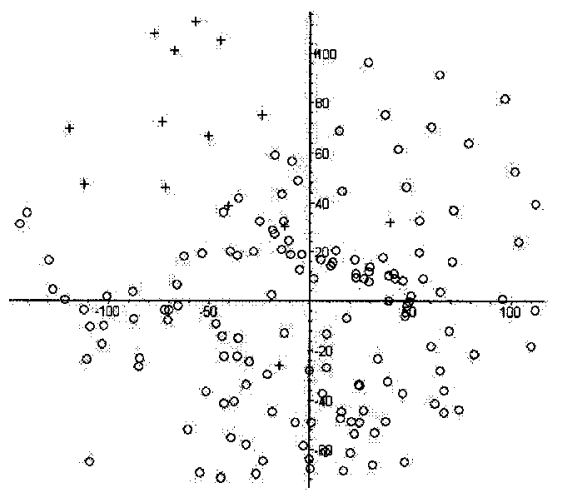

(b)

Figure 4.5: The Figure shows the embedding results for the face database. Figure (a): embedding computed by the embedding algorithm. Figure (b): embedding computed by the projection algorithm. Figure (c): embedding computed by SMACOF.

The embeddings are shown in Figure 4.5(a) to (c). Figure 4.5(a) shows the 


\begin{tabular}{|l|r|r|r|r|}
\hline$n$ & \multicolumn{4}{|c|}{165} \\
\hline & $t$ & $E_{L S M D S}$ & $S(\mathrm{MB})$ & time (sec) \\
\hline Emb. alg. & 174 & $1.17 \cdot 10^{7}$ & 0.070 & 84 \\
\hline Proj. alg. & 48 & $\mathbf{1 . 4 9 \cdot 1 0 ^ { 7 }}$ & 0.052 & 43 \\
\hline SMACOF & 143 & $8.63 \cdot 10^{6}$ & 0.69 & 9 \\
\hline
\end{tabular}

Table 4.2: Quality of embedding for the Yale Face Database.

embedding obtained using the projection algorithm, Figure 4.5(b) shows the result obtained using the embedding algorithm, and Figure 4.5(c) shows the result obtained using the SMACOF algorithm. The points marked as crosses correspond to the expression where the face is lit from the right side only shown on the bottom left of Figure 4.4. We can see that most of the crosses are close in all of the embedding results. This suggests that we can visualize different expressions as clusters of points in the plane.

\subsubsection{Computing a Canonical Form}

The third application we consider is to compute the canonical form of a complete triangular surface. In this application, objects are vertices on a triangular surface and dissimilarities between objects are geodesic distances between the corresponding vertices. Note that in this application, the assumption that the dissimilarity function can be evaluated in constant time does not hold, since it takes $O(n \log n)$ time to compute a geodesic distance [56]. Hence, the running time of the embedding algorithm becomes $O\left(n^{4} \log n+n^{\frac{5}{2}} \log n d t\right)$.

We evaluate the algorithms using the following two experiments. First, we evaluate the algorithms by embedding the surface of the swiss roll dataset into $\mathbb{R}^{2}$. The swiss roll dataset has a non-Euclidean structure, but can be rolled into a planar patch. Due to the complexity of unrolling the swiss roll, this experiment is commonly used to demonstrate the quality of embedding algorithms [22,87,103]. In our experiment, we use the parametric form of the swiss roll surface given by Bronstein et al. [22]: $x=\theta, y=0.51\left(\frac{1}{2.75 \pi}+0.75 \phi\right) \cos (2.5 \phi), z=0.51\left(\frac{1}{2.75 \pi}+0.75 \phi\right) \sin (2.5 \phi)$, where $(\theta, \phi) \in[0,1] \times[0,1]$.

Second, we evaluate the algorithms using the triangular mesh from the Princeton Shape Benchmark [95] shown in Figure 4.9(a) consisting of $n=429$ vertices. We 
refine the mesh using local subdivision of triangles and run the algorithm on different resolutions of the mesh.

In the first (respectively second) experiment, we use geodesic distances on the mesh as dissimilarities and embed the vertices into $\mathbb{R}^{2}$ (respectively $\mathbb{R}^{3}$ ). Table 4.3 (respectively Table 4.4) reports the amount of storage required by the algorithms, the maximum number $t$ of iterations required by the algorithms, the running time of the algorithms, and the error of the embedding computed by the algorithms according to equation (2.2). The time and space complexities of the algorithms is furthermore visualized in Figure 4.6 (respectively Figure 4.7). The $x$-axis of the graph shows the number $n$ of vertices and the $y$-axes show the amount of storage used by the algorithms in $\mathrm{MB}$ and the running times of the algorithms in seconds.

We can see that the amount of storage required by the SMACOF algorithm shown as dashed curve grows significantly faster than the amount of storage required by the embedding algorithm shown as solid curve and the projection algorithm shown as dotted curve. This is to be expected, since the SMACOF algorithm takes $O\left(n^{2}\right)$ storage while the other two algorithms take $O(n)$ storage. Furthermore, the projection algorithm requires only about half of the amount of storage required by the embedding algorithm.

The SMACOF algorithm is fastest and the embedding algorithm is slowest. Note that the difference in running time between the three algorithms is larger than in the previous experiment. The reason is that evaluating the dissimilarity function is slow in this experiment as the running time of the distance function is $O(n \log n)$.

\begin{tabular}{|c|c|c|c|c|c|c|c|c|c|c|c|c|}
\hline \multirow[t]{2}{*}{$\mathrm{n}$} & \multicolumn{4}{|c|}{ Emb. alg. } & \multicolumn{4}{|c|}{ Proj. alg. } & \multicolumn{4}{|c|}{ SMACOF } \\
\hline & $t$ & $E_{L S M D S}$ & $S$ & time & $t$ & $E_{L S M D S}$ & $S$ & time & $t$ & $E_{L S M D S}$ & $S$ & time \\
\hline 100 & 1 & $4.7 \cdot 10^{-27}$ & 0.062 & 12 & 1 & $1.4 \cdot 10^{-27}$ & 0.049 & 8 & 1 & $6.8 \cdot 10^{-28}$ & 0.31 & 2 \\
\hline 250 & 1 & $1.7 \cdot 10^{-26}$ & 0.081 & 149 & 1 & $5.0 \cdot 10^{-26}$ & 0.056 & 108 & 1 & $1.7 \cdot 10^{-27}$ & 1.42 & 26 \\
\hline 500 & 1 & $2.7 \cdot 10^{-025}$ & 0.11 & 1120 & 1 & $1.5 \cdot 10^{-025}$ & 0.068 & 832 & 1 & $1.0 \cdot 10^{-25}$ & 5.20 & 243 \\
\hline 750 & 1 & $5.3 \cdot 10^{-025}$ & 0.11 & 4070 & 1 & $7.2 \cdot 10^{-025}$ & 0.077 & 2633 & $\overline{1}$ & $1.0 \cdot 10^{-24}$ & 11.36 & 793 \\
\hline 1000 & 1 & $9.6 \cdot 10^{-24}$ & 0.17 & 8472 & 1 & $1.2 \cdot 10^{-23}$ & 0.086 & 6462 & 1 & $9.7 \cdot 10^{-24}$ & 19.90 & 1933 \\
\hline
\end{tabular}

Table 4.3: Quality of embedding for the swiss roll. The table shows the maximum number $t$ of iterations required by the LSBFGS quasi-Newton method [73], the embedding error $E_{L S}$ of the computed embedding, the storage use $S$ in $M B$, and the running time in seconds of all three algorithms that were implemented. 


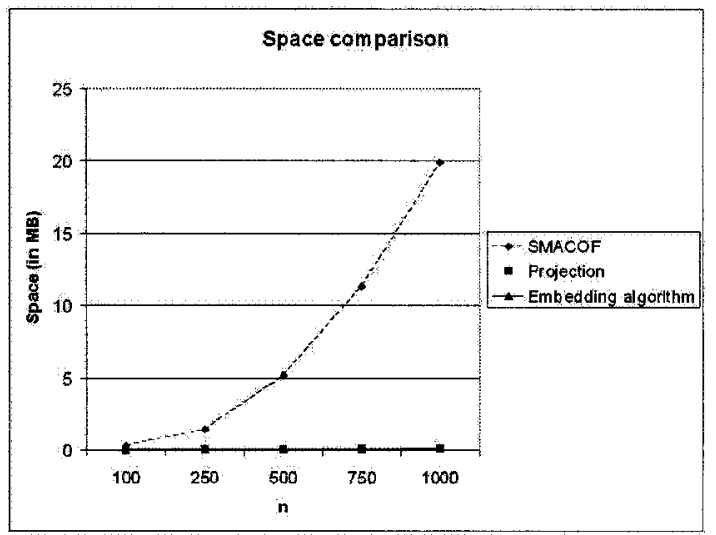

(a)

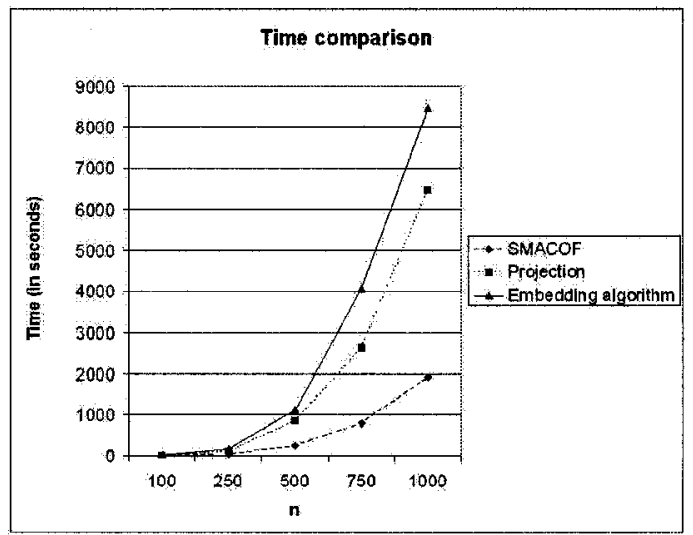

(b)

Figure 4.6: Comparison of time and space used by the embedding algorithm (solid), the projection algorithm (dotted), and the SMACOF algorithm (dashed) for the swiss roll. The number of objects $n$ is shown along the $x$-axis and the space and time requirements are shown along the $y$-axis.

\begin{tabular}{|r|r|r|r|r||r|r|r|r||r|r|r|r|}
\hline $\mathbf{n}$ & \multicolumn{9}{|c||}{ Emb. alg. } & \multicolumn{4}{|c||}{ Proj. alg. } & \multicolumn{4}{|c|}{ SMACOF } \\
\hline & $t$ & $E_{L S M D S}$ & $S$ & time & $t$ & $E_{L S M D S}$ & $S$ & time & $t$ & $E_{L S M D S}$ & $S$ & time \\
\hline 429 & 111 & 190 & 0.12 & 1403 & 91 & 233 & 0.073 & 807 & 99 & 950 & 4.05 & 162 \\
\hline 550 & 143 & 170 & 0.13 & 2894 & 96 & 263 & 0.080 & 1680 & 152 & 126 & 6.45 & 374 \\
\hline 1121 & 143 & 673 & 0.20 & 23720 & 93 & 1550 & 0.11 & 15663 & 128 & 540 & 25.32 & 3150 \\
\hline
\end{tabular}

Table 4.4: Quality of embedding for the Alien. The table shows the maximum number $t$ of iterations required by the LSBFGS quasi-Newton method [73], the embedding error $E_{L S}$ of the computed embedding, and the storage use $S$ of all three algorithms that were implemented.

An image of the swiss roll containing 500 vertices as well as the embeddings computed using the tested algorithms are shown in Figure 4.8. Note that all of the results are similar except for rotations.

For the Alien dataset $(n=429)$, the embedding obtained using the projection algorithm is shown in Figure 4.9(b), the embedding obtained using the embedding algorithm is shown in Figure 4.9(c), and the embedding obtained using the SMACOF algorithm is shown in Figure 4.9(d). We can see that all of the embeddings are similar. 


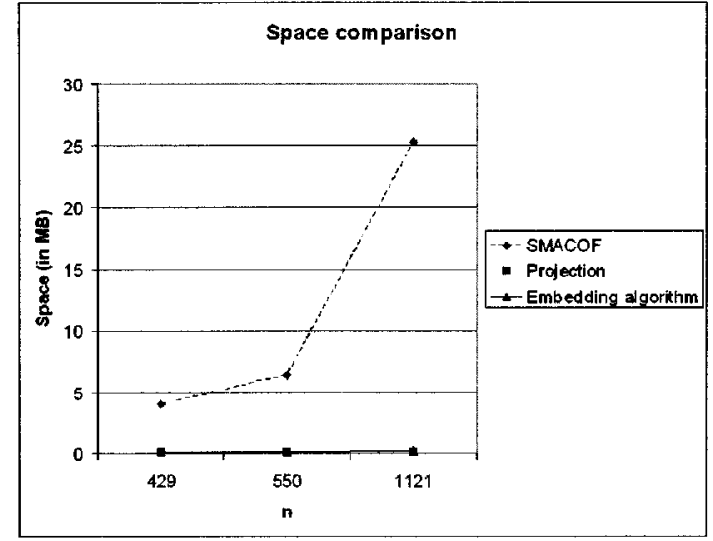

(a)

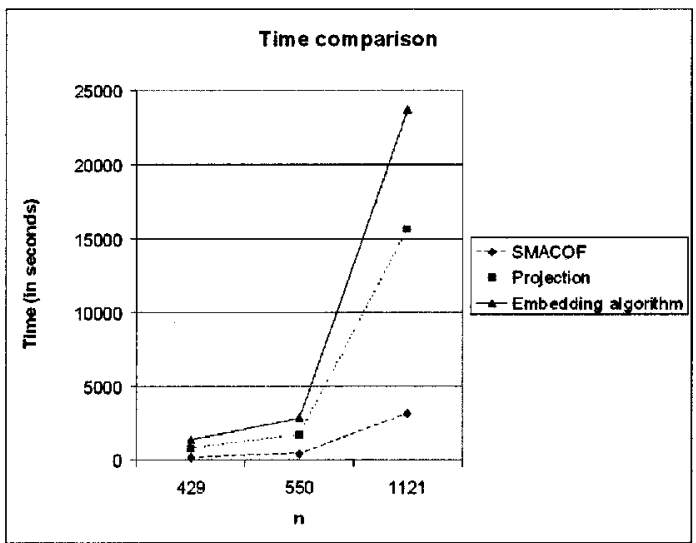

(b)

Figure 4.7: Comparison of time and space used by the embedding algorithm (solid), the projection algorithm (dotted), and the SMACOF algorithm (dashed) for the Alien. The number of objects $n$ is shown along the $x$-axis and the space and time requirements are shown along the $y$-axis.

\subsubsection{Summary}

We conclude that both the embedding algorithm and the projection algorithm can compute an embedding of lower quality than the SMACOF embedding using significantly less storage. In most of our experiments, the error of the embedding computed using the embedding algorithm is about twice the error of the embedding computed using SMACOF. As expected, the embedding algorithm yields an embedding of higher

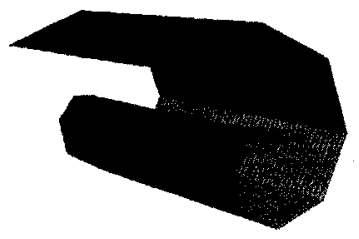

(a)

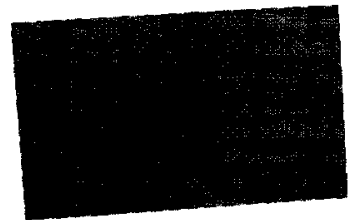

(b)

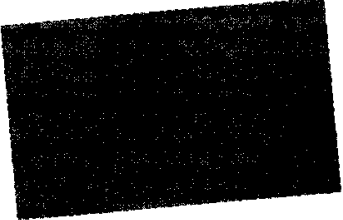

(c)

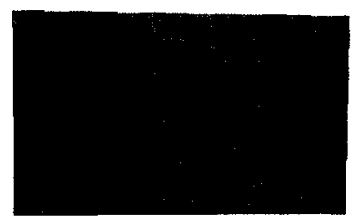

(d)

Figure 4.8: The Figure shows the swiss roll and its embeddings. Figure (a): swiss roll with $n=500$ vertices. Figure (b): embedding computed by the embedding algorithm. Figure (c): embedding computed by projection algorithm. Figure (d): embedding computed by SMACOF. Note that the results shown in (b)-(d) are similar except for rotations. 


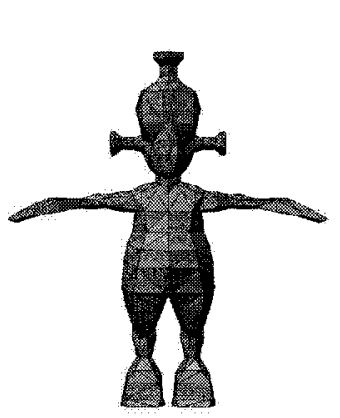

(a)

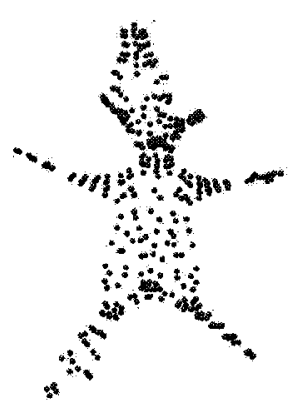

(b)

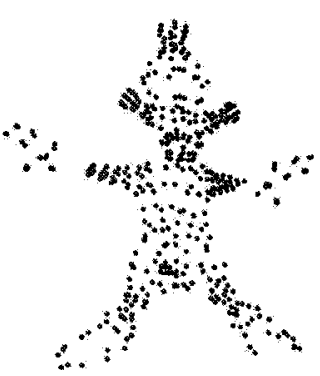

(c)

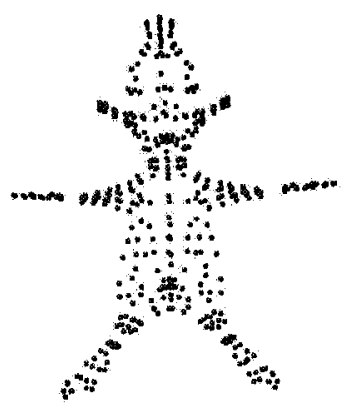

(d)

Figure 4.9: Alien model used for evaluation of results. Figure (a) shows the original model, Figure (b) shows the embedding using the projection algorithm, Figure (c) shows the embedding using the embedding algorithm, and Figure (d) shows the embedding using SMACOF algorithm.

quality than the projection algorithm.

Due to its linear space requirement, the embedding algorithm can be applied to compute embeddings for large datasets where storing a full dissimilarity matrix is no longer feasible as shown using the gamma telescope dataset. The embedding algorithm is especially useful to compute a low-dimensional embedding of a large dataset where the dissimilarity function can be evaluated fast. In this case, the embedding algorithm was shown to outperform the SMACOF algorithm in terms of running time. If the evaluation of the dissimilarity function is a large constant or a function whose running time grows as a function of $n$, then the embedding algorithm is slower than the SMACOF algorithm.

\subsection{Conclusions}

This chapter considers the problem of finding a set of points in low-dimensional space so that the distances between points are preserved. Although MDS or principal coordinate analysis are powerful techniques for this purpose, they require quadratic space complexity. We have proposed a linear-space algorithm assuming that the dissimilarity for any pair of objects can be computed in constant time using a function. Our experiments show that when computing canonical representations of triangular 
manifolds the quality of the embedding computed using the proposed linear-space algorithm is only slightly lower than the quality of the embedding computed using the SMACOF algorithm. However, the space requirement of the embedding algorithm was shown to be significantly lower than the space requirement of the SMACOF algorithm.

An important open question is to find an algorithm to compute an embedding in a low-dimensional space that has provably low distortion using linear space. 


\title{
Chapter 5
}

\section{Posture Invariant Correspondence}

\author{
A kitten is so flexible that she is almost double; the hind parts are equivalent \\ to another kitten with which the forepart plays. She does not discover that \\ her tail belongs to her until you tread on it. \\ HENRY DaVid THOREAU (1817-1862)
}

This chapter presents an approach to find dense point-to-point correspondences between two deformed surfaces corresponding to different postures of the same nonrigid object in a fully automatic way. The approach requires no prior knowledge about the shapes being registered or the initial alignment of the shapes. We consider surfaces that are represented by possibly incomplete triangular meshes. We model the deformations of an object as isometries. To solve the correspondence problem, our approach maps the intrinsic geometries of the surfaces into a low-dimensional Euclidean space via multi-dimensional scaling as in Chapter 3. This results in postureinvariant shapes that can be registered using rigid correspondence algorithms. This work is published in the International Journal of Shape Modeling [110].

\subsection{Introduction}

We consider the problem of finding dense point-to-point correspondences between two deformed surfaces $S^{(0)}$ and $S^{(1)}$ corresponding to different postures of the same 
non-rigid object. That is, given a position $x^{(0)}$ on $S^{(0)}$, we aim to find the position $x^{(1)}$ on $S^{(1)}$ that corresponds to the same intrinsic location on $S^{(1)}$ as does $x^{(0)}$ on $S^{(0)}$. If the position $x^{(1)}$ is absent on $S^{(1)}$ due to incomplete data, no correspondence is found for $x^{(0)}$. Finding dense point-to-point correspondences between two deformed surfaces is a key problem in various applications such as mesh deformation and animation [5], shape registration [69], object recognition [49], and mesh parameterization $[61,89]$. The $3 \mathrm{D}$ models used in these applications usually come from digitizing real-world objects from a discrete set of measurements using a 3D laser-range scanner or image-based reconstruction. Therefore, the reconstructed surfaces are often incomplete.

The main difficulty in finding point-to-point correspondences is that local regions on the surface are often not distinctive. Hence, finding the correspondence for all object points corresponds to searching a large set of candidate correspondences. Previous methods to find point-to-point correspondences for deformable surfaces either restrict the search space using prior knowledge about the objects being registered [2] or use probabilistic methods to solve the problem [4] which has the drawback of producing inaccurate correspondences.

The method proposed in this chapter considers surfaces that are represented by possibly incomplete triangular meshes. We model deformations of an object as isometries. We aim to find dense point-to-point correspondences between two incomplete triangular surfaces $S^{(0)}$ and $S^{(1)}$ consisting of $n^{(0)}$ and $n^{(1)}$ vertices, respectively, using a fully automatic correspondence algorithm that does not assume knowledge about markers or template shapes.

We propose an approach to solve the registration problem that can be viewed as an extension of Jain et al.'s approach [50] and that is related to Bronstein et al.'s approach [20]. Both the approach by Jain et al. and the approach by Bronstein et al. have only been used to compute the correspondence between a small set of samples consisting of no more than 3000 vertices. This is due to the high complexity of the algorithms. We extend the approach of Jain et al. by applying a course-to-fine strategy, thereby making the approach useful for real-life data sets containing tens of thousands of vertices.

Our approach consists of three main steps. First, we compute uniformly distributed 
sample sets $P^{(r)}$ containing $n^{\prime(r)}$ vertices from $S^{(r)}$ for $r=0,1$. We compute the geodesic distance $\delta_{i, j}$ between the vertices $p_{i}$ and $p_{j}$ for $i, j \in P^{(r)}$ using the fast marching technique introduced by Kimmel and Sethian [56]. Furthermore, we compute confidence values $\omega_{i, j}=1-\frac{m_{i, j}^{h}}{m_{i, j}}$, where $m_{i, j}$ is the number of edges on the geodesic path computed by the fast marching technique from $p_{i}$ to $p_{j}$ and where $m_{i, j}^{h}$ is the number of edges tracing a hole of $S^{(r)}$ on the geodesic path from $p_{i}$ to $p_{j}$. We say that an edge traces a hole of $S^{(r)}$ if the edge crosses a triangle that contains at least one vertex on the hole. We use the geodesic distances $\delta_{i, j}$ as dissimilarities and the confidence values $\omega_{i, j}$ as weights to embed the samples $P^{(r)}$ of the manifold $S^{(r)}$ via multi-dimensional scaling. Let $X^{(r)}$ denote the embedding. The approach embeds $P^{(r)}$ into a low-dimensional Euclidean space $\mathbb{R}^{k}$ using least-squares MDS.

Second, we compute the rigid correspondence between the posture-invariant canonical forms. Our approach employs the Hungarian method [80] for this computation. The Hungarian method finds a maximum weight matching in a weighted bipartite graph.

Third, all vertices of $S^{(r)} \backslash P^{(r)}$ are projected to the embedding space. The approach finds the correspondence of the projected vertices by evaluating an approximating thin-plate spline mapping the embedding $X^{(0)}$ to $X^{(1)}$.

\subsection{Correspondence via Least-Squares Multi- Dimensional Scaling}

We find the point-to-point correspondence between vertices on $S^{(0)}$ and $S^{(1)}$, respectively, using a method that extends the work of Jain et al. [50]. One limitation of Jain et al.'s approach is its quadratic space complexity. This complexity stems from the computation of the canonical form and limits the scalability of the approach. The models considered for Jain et al.'s experiments all have at most 250 vertices. We overcome this limitation and make the approach applicable to real-world data consisting of tens of thousands of vertices by taking a coarse-to-fine approach.

Our algorithm is summarized in Algorithm 3 and outlined in more detail in 
Section 5.3. The input of the algorithm consists of two possibly incomplete triangular manifolds $S^{(0)}$ and $S^{(1)}$ consisting of $n^{(0)}$ and $n^{(1)}$ vertices, respectively.

\subsection{Description of the Algorithm}

In this section, we describe each step of the approach in detail. First, the approach computes a small number of uniformly distributed sample points $P^{(0)}$ and $P^{(1)}$ on $S^{(0)}$ and $S^{(1)}$, respectively, using Voronoi sampling (see Section 2.5 ).

Second, we establish a coarse correspondence between the sample points $P^{(0)}$ and $P^{(1)}$ as described in Section 5.3.1. Third, we establish the fine correspondence, that is, the correspondences between vertices in $S^{(0)} \backslash P^{(0)}$ and $S^{(1)} \backslash P^{(1)}$. This step is described in Section 5.3.2.

\subsubsection{Coarse Correspondence}

We establish the coarse correspondence, which is the correspondence between the two sample sets, using a variation of the approach introduced by Jain et al. [50]. For the sample set $P^{(r)}, r=0,1$, we compute the pairwise geodesic distances $\delta_{i, j}$ on $S^{(r)}$ between the vertices $p_{i}$ and $p_{j}$ for $i, j \in P^{(r)}$ using the fast marching technique [56]. In fact, due to the close relationship between the fast marching technique and farthest point sampling, we compute $\delta_{i, j}$ at the same time as we compute the sample points. We do this by storing all of the geodesic distances between each sample point $p_{i}$ and all of the other vertices of $S^{(r)}$. We also compute confidence values $\omega_{i, j}$ as approximate fraction of the geodesic path between $p_{i}$ and $p_{j}$ that does not trace a hole of $S^{(r)}$. We use confidence values $\omega_{i, j}=1-\frac{m_{i, j}^{h}}{m_{i, j}}$, where $m_{i, j}$ is the number of edges on the geodesic path from $p_{i}$ to $p_{j}$ on $S^{(r)}$ and where $m_{i, j}^{h}$ is the number of edges tracing a hole of $S^{(r)}$ on the geodesic path on $S^{(r)}$ from $p_{i}$ to $p_{j}$. Chapter 3 shows that this choice of confidence values yields MDS embeddings that represent well the geodesic distances on $S^{(r)}$.

We embed the $n^{\prime(r)}$ sample vertices $P^{(r)}=\left\{p_{1}^{(r)}, \ldots, p_{n^{\prime}(r)}^{(r)}\right\}$ into $\mathbb{R}^{k}$ for a constant embedding dimension $k$ using least-squared MDS as discussed in Section 2.3. We 


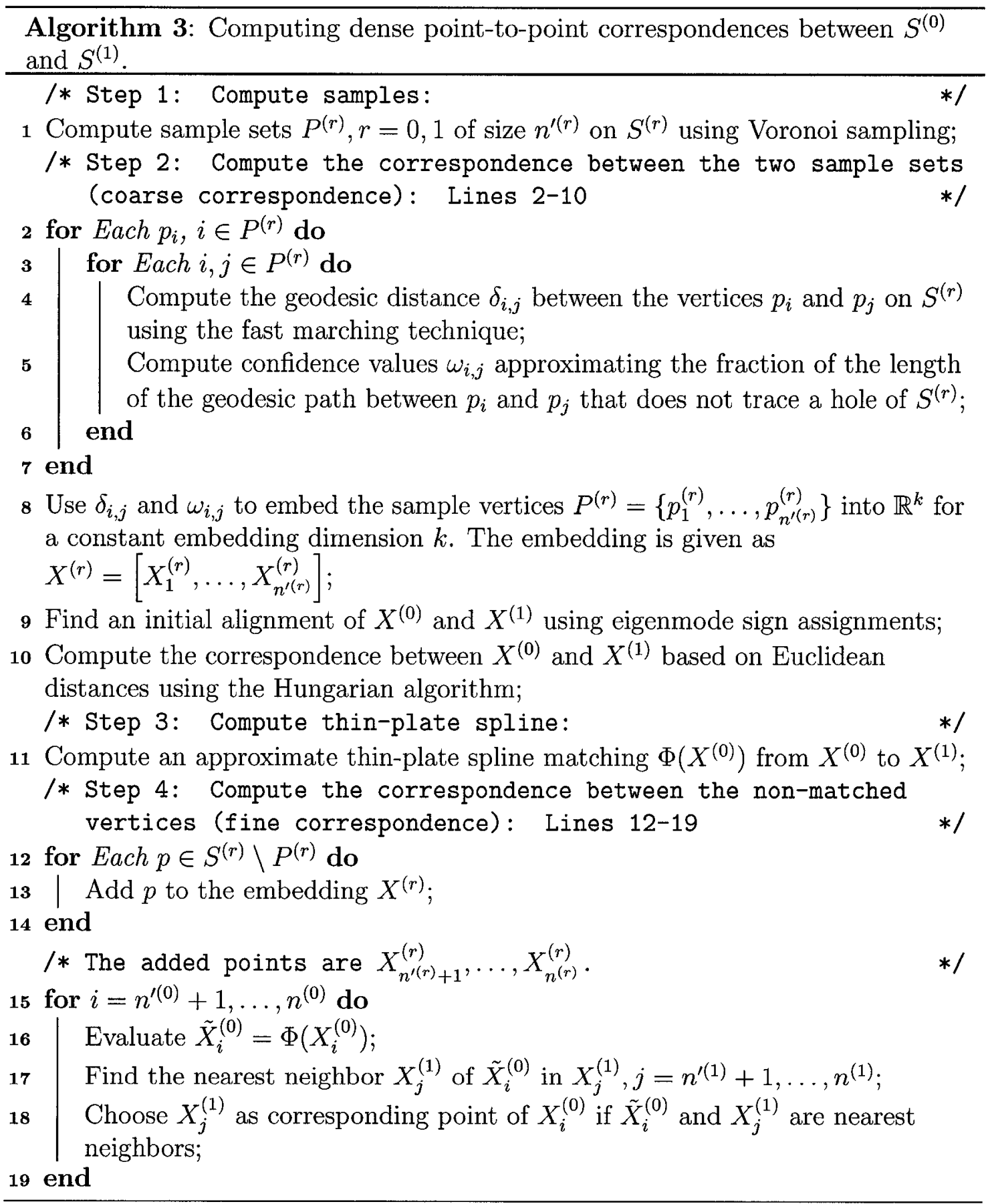


perform least-squares MDS with the geodesic distances $\delta_{i, j}$ as dissimilarities and the confidence values $\omega_{i, j}$ as weights to embed the samples $P^{(r)}$ of the manifold $S^{(r)}$ to an embedding configuration $X^{(r)}$ in $\mathbb{R}^{k}$. The configurations $X^{(r)}$ have the property that Euclidean distances in embedding space approximate well the geodesic distances on $S^{(r)}$ according to $E_{L S}$. Hence, $X^{(r)}$ is called the bending invariant form or canonical form [34].

Since the embeddings $X^{(0)}$ and $X^{(1)}$ are approximately bending invariant, we can find correspondences between the sample sets by rigid registration of the canonical forms. A similar approach has been implemented by Jain et al. [50]. As the canonical forms $X^{(r)}$ are invariant with respect to rotation, translation, and reflection [28, Chapter 1], we need to consider multiple alignments of $X^{(0)}$ and $X^{(1)}$. We do this as follows. First, we align both $X^{(0)}$ and $X^{(1)}$ by the eigenvectors of their respective data covariance matrices. Second, we compute an optimal rigid correspondence using the Hungarian method [80] for each sign assignment of the eigenvectors. Third, after computing the $2^{k}$ different rigid correspondences, we choose the one that yields the lowest cost.

In the first step, we learn the two covariance matrices of the embeddings $X^{(0)}$ and $X^{(1)}$. We then compute the eigenvectors of the covariance matrices. Let the eigenvectors be sorted in decreasing order with respect to their corresponding eigenvalues. We align the embeddings $X^{(0)}$ and $X^{(1)}$, such that the coordinate axes correspond to the directions of the eigenvectors in order. That is, we align the coordinate axes with the directions of the largest data variance of the embeddings. Since $X^{(r)}$ is invariant with respect to reflection, there are $2^{k}$ relative alignments of $X^{(0)}$ and $X^{(1)}$ that preserve the order of the eigenvectors. These relative alignments are called the sign assignments of the eigenvectors in the following.

In the second step, we compute an optimal rigid correspondence for a given sign assignment of the eigenvectors. Without loss of generality assume that $n^{(0)} \leq n^{(1)}$. To compute the optimal rigid correspondence for a given sign assignment, we aim to find an assignment function $a(i)$ that assigns exactly one point $X_{a(i)}^{(1)}$ to every point $X_{i}^{(0)}$, such that $E_{H}=\sum_{i=1}^{n^{\prime(0)}} d\left(X_{i}^{(0)}, X_{a(i)}^{(1)}\right)$ is minimized, where $d$ denotes the Euclidean distance in $\mathbb{R}^{k}$. This problem can be viewed as an assignment problem 
and it can be solved by finding a maximum-weight matching in a bipartite graph as follows. Let the bipartite graph be the complete bipartite graph on the two vertex sets $X^{(0)}$ and $X^{(1)}$. Let the edge between the vertices $X_{i}^{(0)}$ and $X_{j}^{(1)}$ of the bipartite graph have weight $\frac{1}{d\left(X_{i}^{(0)}, X_{j}^{(1)}\right)}$. Finding the maximum weight matching in this graph yields the sought assignment $a(i)$. We compute the maximum weight matching in cubic time using the Hungarian algorithm [80]. After assigning a point $X_{a(i)}^{(1)}$ to a point $X_{i}^{(0)}$, we can measure $e=d\left(X_{i}^{(0)}, X_{a(i)}^{(1)}\right)$. As $e$ is measured as Euclidean distance in embedding space, $e$ is an approximation of the error made by corresponding $X_{i}^{(0)}$ to $X_{a(i)}^{(1)}$ measured as geodesic distance on $S^{(0)}$ or $S^{(1)}$.

To eliminate erroneous matchings that assign a point $X_{a(i)}^{(1)}$ to a point $X_{i}^{(0)}$ although the part corresponding to $p_{i}^{(0)}$ is missing in $S^{(1)}$, we eliminate matching points where $e$ is larger than the sampling resolution along the mesh.

Note that any rigid or almost rigid registration algorithm can be used to find the coarse correspondence. Jain et al. [50] use a modified iterative closest point method for this step. As there is a one-to-one correspondence between $P^{(r)}$ and $X^{(r)}$, the correspondences of the points $P^{(0)}$ and $P^{(1)}$ directly follow.

Finally, in the third step, we choose as coarse correspondence the alignment of $X^{(0)}$ and $X^{(1)}$ that yields the lowest cost $E_{H}$.

\subsubsection{Fine Correspondence}

This section describes how to compute the fine correspondence given the coarse correspondence. We model the deformation of $X^{(0)}$ to $X^{(1)}$ using an approximate thinplate spline. The following description is due to Dryden and Mardia [31]. Denote the matrix of points of $X^{(0)}$ that have a valid corresponding point in $X^{(1)}$ by $T=\left[\vec{t}_{1} \vec{t}_{2} \ldots \vec{t}_{l}\right]$ with $l \leq n^{\prime(0)}$. Furthermore, denote the matrix of points of $X^{(1)}$ corresponding to $T$ in that order by $Y=\left[\vec{y}_{1} \vec{y}_{2} \ldots \vec{y}_{l}\right]$. The thin-plate spline deformation is $\Phi(\vec{t})=$ $\vec{c}+A \vec{t}+W^{T} s(\vec{t})$, where $\vec{c}$ is a $k$-dimensional vector, $A$ is a $k \times k$ matrix, $W$ is a $l \times k$ matrix, and $s(\vec{t})=\left[\phi\left(\vec{t}-\vec{t}_{1}\right) \ldots \phi\left(\vec{t}-\vec{t}_{l}\right)\right]^{T}$ is a $l$-dimensional vector with

$$
\phi(\vec{t})=\left\{\begin{array}{l}
\|\vec{t}\|^{2} \log \|\vec{t}\|,\|\vec{t}\|>0, \\
0, \text { otherwise }
\end{array} .\right.
$$


We find $\Phi(\vec{t})$ by solving the linear system of equations

$$
\left[\begin{array}{ccc}
S+\lambda I & \mathbf{1} & T^{T} \\
\mathbf{1}^{T} & 0 & 0 \\
T & 0 & 0
\end{array}\right]\left[\begin{array}{c}
W \\
c^{T} \\
A^{T}
\end{array}\right]=\left[\begin{array}{c}
Y^{T} \\
0 \\
0
\end{array}\right],
$$

where $I$ is the identity matrix, $S_{i, j}=\phi\left(\vec{t}_{i}-\vec{t}_{j}\right), 1$ is an $l \times 1$ vector containing 1 at each position, and $\lambda$ is a constant. Following Jain et al. [50], we choose $\lambda$ as the median distance between matching point pairs. The approximate thin-plate spline gives us a way of transforming any point from the embedding $X^{(0)}$ to the embedding $X^{(1)}$.

We establish the fine correspondence, that is, the correspondences between vertices in $S^{(0)} \backslash P^{(0)}$ and $S^{(1)} \backslash P^{(1)}$. To compute the correspondence in embedding space, we add all of the points in $S^{(r)} \backslash P^{(r)}$ to the embedding $X^{(r)}$ for $r=0,1$ as outlined in Section 3.2. To add $p_{n^{\prime}(r)+j}^{(r)}, j>0$ to the least-squares MDS embedding, we compute the geodesic distances $\delta_{n^{\prime(r)}+j, 1}, \ldots, \delta_{n^{\prime}(r)+j, n^{\prime}(r)}$ as dissimilarities and the confidence values $\omega_{n^{\prime(r)}+j, 1}, \ldots, \omega_{n^{\prime(r)}+j, n^{\prime(r)}}$ as weights as before. We then find a position $X_{n^{\prime(r)}+j}^{(r)}$ such that

$$
E_{L S}^{*}=\sum_{i=1}^{n^{\prime(r)}} \omega_{n^{\prime(r)}+j, i}\left(\delta_{n^{\prime(r)}+j, i}-d_{n^{\prime(r)}+j, i}(X)\right)^{2}
$$

is minimized. Note that the geodesics and weights do not need to be recomputed, since we computed and stored those values during farthest point sampling. Let the embedded points be denoted by $X_{n^{\prime(r)}+1}^{(r)}, \ldots, X_{n^{(r)}}^{(r)}$. Once the embedded points are known, we use the approximate thin-plate spline to find the correspondences in bending invariant space. We first compute $\tilde{X}_{i}^{(0)}=\Phi\left(X_{i}^{(0)}\right)$ for $i=n^{(0)}+1, \ldots, n^{(0)}$. We choose $X_{j}^{(1)}, j=n^{\prime(1)}+1, \ldots, n^{(1)}$ as corresponding point of $X_{i}^{(0)}$ if $\tilde{X}_{i}^{(0)}$ and $X_{j}^{(1)}$ are nearest neighbors.

As in the case of coarse correspondences, we eliminate matching points where the Euclidean distance between the points is larger than the sampling resolution along the mesh to reduce the number of erroneous matchings.

Note that this heuristic algorithm is similar, albeit more general than the work by Jain et al. [50]. Jain et al. use a slight modification of step 2 described in Algorithm 3 to register two surfaces $S^{(0)}$ and $S^{(1)}$. By deriving a coarse-to-fine approach for the 
correspondence problem using canonical forms, we make the use of canonical forms applicable to real-life data sets with tens of thousands of vertices. The reason is that the coarse-to-fine approach overcomes the quadratic time and storage complexity of the algorithm. A detailed analysis of the presented algorithm follows.

\subsection{Analysis of the Algorithm}

We analyze the total space and time complexity of the algorithm by analyzing each step given in Algorithm 3. Let $n=\max \left(n^{(0)}, n^{(1)}\right), n^{\prime}=\max \left(n^{(0)}, n^{\prime(1)}\right)$. The Voronoi sampling in step 1 of the algorithm takes $O\left(n^{\prime} n \log n\right)$ time and space. We can store the dissimilarities and weights computed during the execution of the sampling algorithm. That way, all the weights and dissimilarities needed during the execution of the algorithm are precomputed.

The coarse correspondence in step 2 of the algorithm takes $O\left(t n^{2}\right)$ time to compute the canonical forms of $P^{(r)}$, where $t$ is the maximum number of iterations required by the quasi-Newton algorithm. Trying all align assignments of the eigenmodes and executing the Hungarian method each time takes $O\left(2^{k} n^{\prime 3}\right)$ time. Hence, the total time in step 2 is $O\left(n^{\prime 2}\left(t+2^{k} n^{\prime}\right)\right)$.

Computing the approximate thin-plate spline in step 3 of the algorithm requires solving a linear system of equations of size $n^{\prime}+k+1$. This takes $O\left(\left(n^{\prime}+k\right)^{3}\right)$ time [84, Chapter 2].

Finally, step 4 of the algorithm takes $O(t k n)$ time to project all the points in $S^{(r)} \backslash P^{(r)}$ to the embedding $X^{(r)}$, where $t$ is the maximum number of iterations required by the quasi-Newton algorithm. This holds since all the weights and dissimilarities are precomputed. Evaluating the RBF for all of the points takes $O(n k)$ time. It remains to find the nearest neighbor in $X^{(1)}$ for each point in $\tilde{X}^{(0)}$. We use a three-dimensional $\mathrm{kd}$-tree on $X^{(1)}$ to perform this step more efficiently. A kd-tree is a space-partitioning data structure that repeatedly splits space using orthogonal hyperplanes. Each node of the tree is three-dimensional; each non-leaf node generates a splitting hyperplane that divides space into two subspaces and each leaf node corresponds to one of the vertices of $X^{(1)}$. The tree can be built in $O(n \log n)$ time and uses $O(n)$ storage. Using 
the kd-tree, it takes $O(n \sqrt{n})$ to find all the nearest neighbors [30, Chapter 5.2]. The total time consumed by step 4 is therefore $O(n(t k+\sqrt{n}))$.

Hence, the total time complexity of the algorithm is $O\left(n^{\prime} n \log n+n^{2}\left(t+2^{k} n^{\prime}\right)+\right.$ $\left.\left(n^{\prime}+k\right)^{3}+n(t k+\sqrt{n})\right)$.

Since $k$ is a constant and since in the average case $n^{\prime}<<n$, the running time becomes $O(n \sqrt{n})$. The total space complexity is $O\left(n^{\prime 2}+n\right)$, which is $O(n)$ for the average case $n^{\prime}<<n$. Hence, we reduced the average asymptotic space and time requirement compared to the algorithm suggested by Jain et al. [50], thereby making the technique scalable.

Note that the running time of our algorithm for an average case is dominated by finding all the nearest neighbors. We can improve the asymptotic running time of this step, thereby improving the average asymptotic running time of our algorithm. The exact neighbors in $\mathbb{R}^{k}$ can be found in $O\left(k^{O(1)} \log n\right)$ time $[27,76]$. This reduces the running time of our algorithm on average to $O\left(n k^{O(1)} \log n\right)$. However, the space requirement of the data structures used to answer the nearest neighbor queries grows exponentially in $k$.

If we allow approximate nearest neighbors, the time and space complexity of our algorithm can be improved. Let the nearest neighbor of point $\tilde{X}_{i}^{(0)}$ be denoted by $X_{j}^{(1)}$. An approximate nearest neighbor of $\tilde{X}_{i}^{(0)}$ with approximation constant $1+\epsilon, \epsilon>0$ is defined as a point in $X^{(1)}$ with distance at most $(1+\epsilon) d\left(\tilde{X}_{i}^{(0)}, X_{j}^{(1)}\right)$ from $\tilde{X}_{i}^{(0)}$. An approximate nearest neighbor of $\tilde{X}_{i}^{(0)}$ can be found in $O\left(\left(\frac{k \log n}{\epsilon}\right)^{O(1)}\right)$ time using $O\left(n^{1 / \epsilon^{O(1)}}\right)$ space $[47,48]$. Using this technique reduces the average running time of our algorithm to $O\left(n\left(\frac{k \log n}{\epsilon}\right)^{O(1)}\right)$.

In our implementation, we use a kd-tree to perform the nearest neighbor search due to the simplicity of its implementation.

\subsection{Experimental Results}

We implemented and tested the algorithm using models from the CAESAR database [85], the McGill 3D shape benchmark [117], and the Princeton Shape Benchmark [95]. We further used models with known ground truth to evaluate the quality of the approach. 
We first describe the experiment that evaluates the quality of our approach. We chose a model of an alien from the Princeton Shape Benchmark, subdivided the model to obtain a high resolution mesh, and animated the model to obtain multiple postures with known correspondences using the automatic technique by Baran and Popović [10]. Figure 5.1 illustrates the experiment. The three postures of the alien used to conduct the experiment consist of 6858 vertices and are shown in the first row of the figure. The second row of the figure shows the 2500 sample vertices found on each model using farthest point sampling. The third row of the figure shows the canonical forms of the sample points. The fourth row shows the full fine correspondence found by the algorithm. We found the correspondences between posture (a) and postures (b) and (c) in this experiment. Each vertex is assigned a unique color between red and green in posture (a). The corresponding points in postures (b) and (c) are then displayed using the same color in row four of Figure 5.1. We can see that a visually pleasing correspondence is found.

We compare the correspondences found by our algorithm to the ground truth by computing the geodesic distances between the correspondence found by the algorithm and the true correspondence for each vertex. We measure the error in correspondence as the number of edges along the shortest path between the correspondence found by the algorithm and the true correspondence. Since the algorithm rejects erroneous matchings automatically as outlined in Section 5.3.1, some points do not obtain a correspondence. We do not assign an error to rejected correspondences. When registering posture (a) and posture (b), 1935 correspondences are rejected as erroneous. When registering posture (a) and posture (c), 1842 correspondences are rejected as erroneous. A histogram of the error encountered is shown in Figure 5.2. The histogram shows two different data sets: the set of errors when corresponding posture (a) to posture (b) is shown in black and the set of errors when corresponding posture (a) to posture (c) is shown in grey. Nearly all of the correspondences found by our algorithm are accurate within a distance of two edge lengths. This shows that the approach taken in this chapter yields correspondences of high quality. 

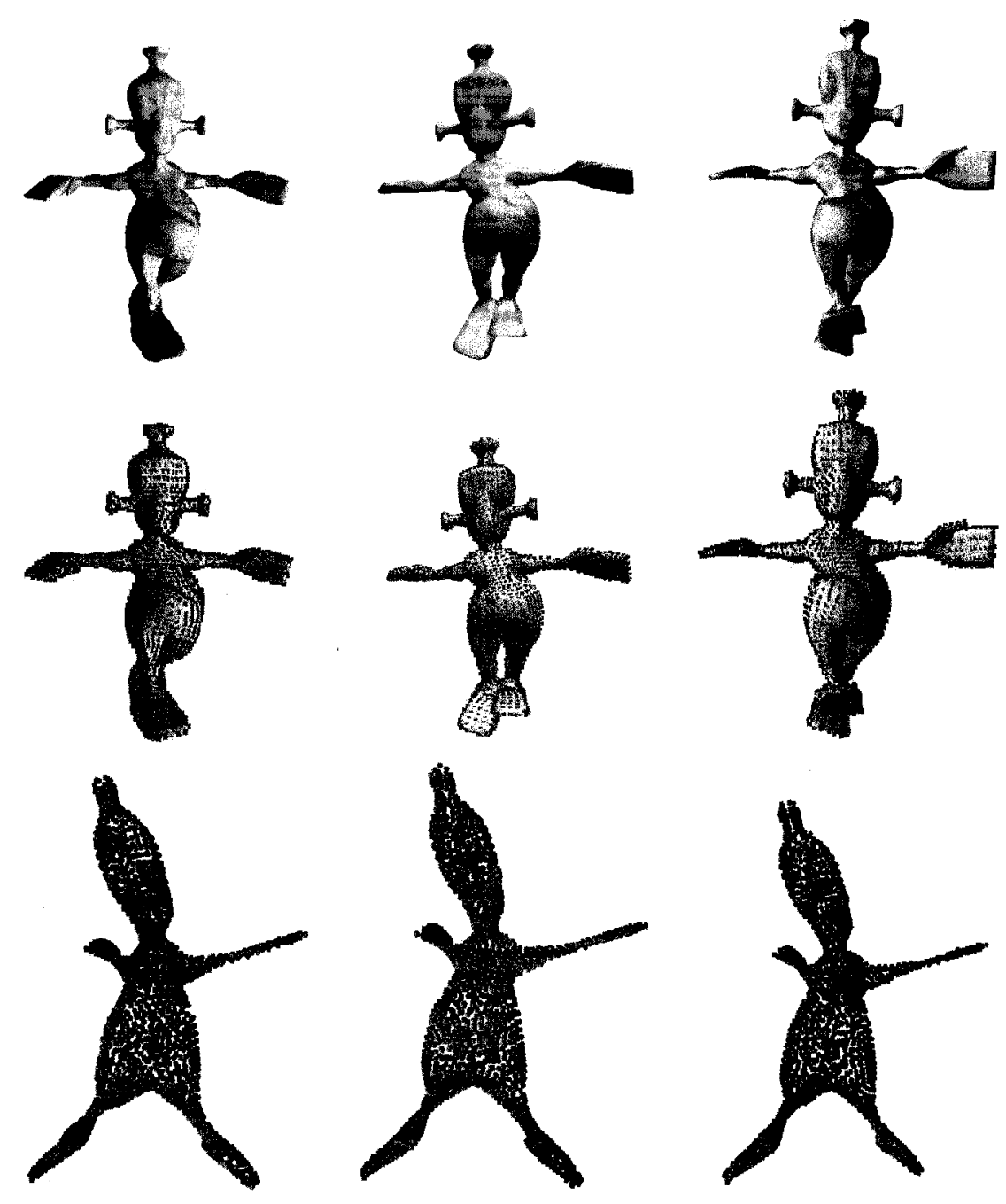

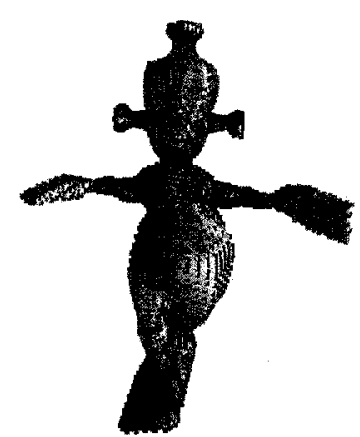

(a)

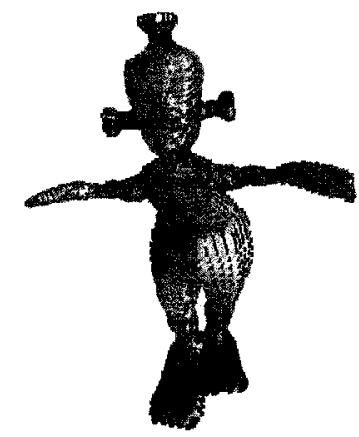

(b)

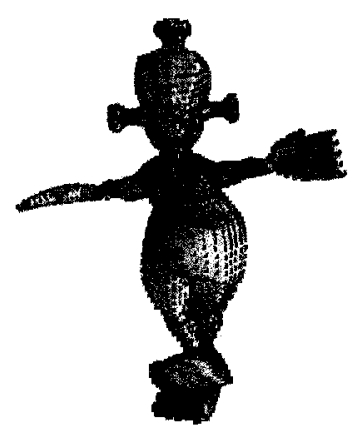

(c)

Figure 5.1: Models of alien in postures (a) to (c) with known ground truth. The first row shows the models, the second row shows the sample points used to find the coarse correspondence, the third row shows the canonical forms of the sample points, and the fourth row shows the color-coded correspondence. 
For some poses of the alien model, the correspondences found are erroneous due to symmetry alignment. A case for which this problem occurs is when registering the posture shown in Figure 5.1 (a) with the posture shown in Figure 5.3. In this case, the left side of the posture shown in Figure 5.1 (a) is found to correspond to the right side of the posture shown in Figure 5.3 and vice versa. To illustrate this, Figure 5.3 shows a true correspondence we aim to find in green and the correspondence found by the algorithm in red. The problem of symmetric alignments is a limitation of our approach and it is discussed in more detail below.

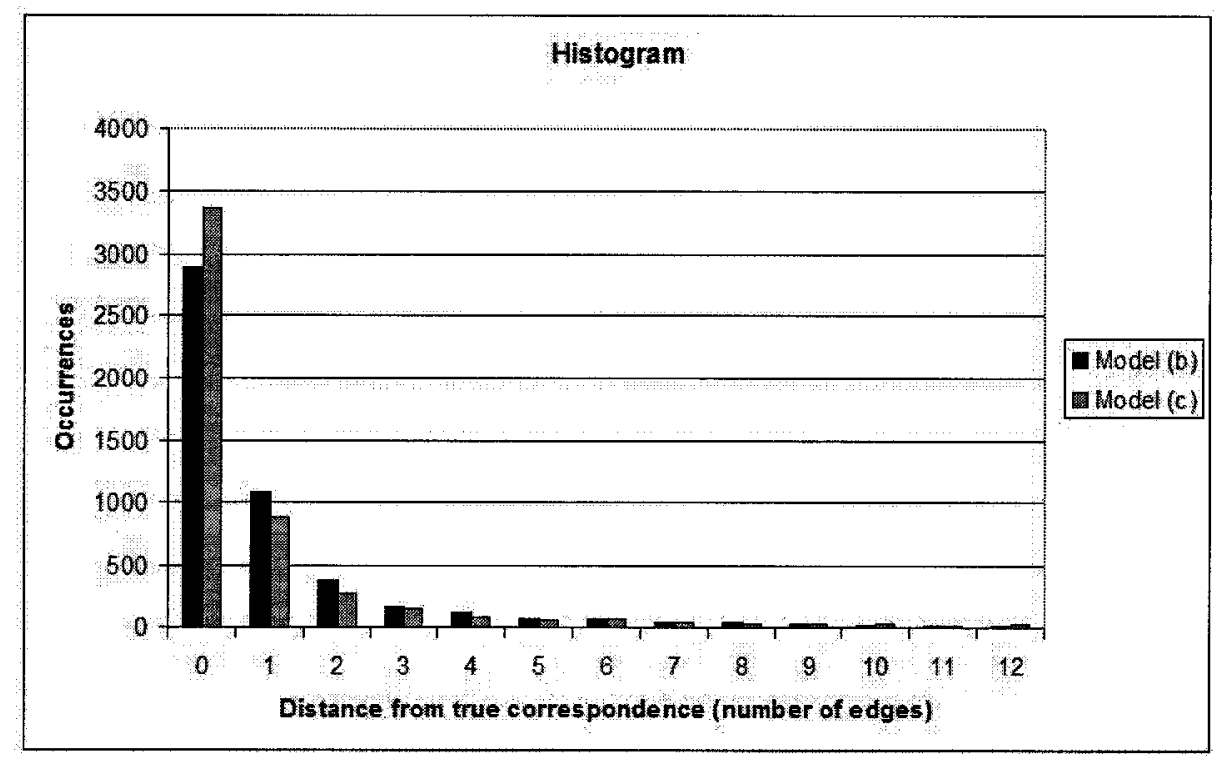

Figure 5.2: Histogram of errors made by correspondence algorithm. The black columns show the histogram of errors when corresponding posture (a) to posture (b). The grey columns show the histogram of errors when corresponding posture (a) to posture (c).

We further conducted two experiments with incomplete real-life data sets. The first articulated model we experimented with is the model of a teddy bear from the McGill 3D shape benchmark. Figure 5.4(a) shows the first model consisting of 21338 vertices, 2000 of which were chosen as sample points. Figure 5.4(b) shows the second model consisting of 25658 vertices, 2050 of which were chosen as sample points. Note that both models contain many small holes. Figure 5.5 shows the aligned canonical forms of the two bears. The canonical forms are almost rigid transformations. The 


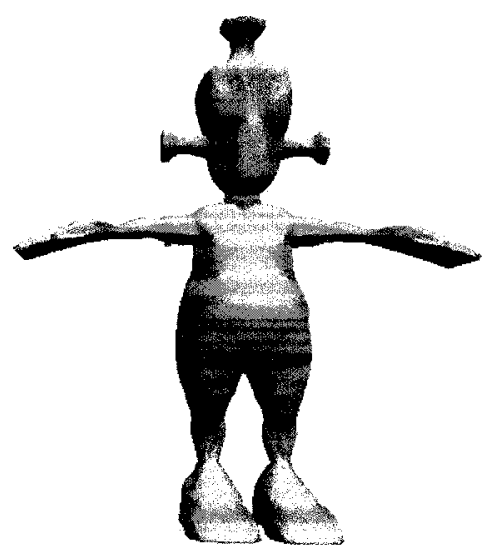

Figure 5.3: Erroneous correspondence due to symmetry problems.

correspondence computed by our algorithm is visualized in Figure 5.6. We visualize the result by assigning a unique color to each vertex on the first model and by drawing vertices on the second model in same color as their corresponding vertices.

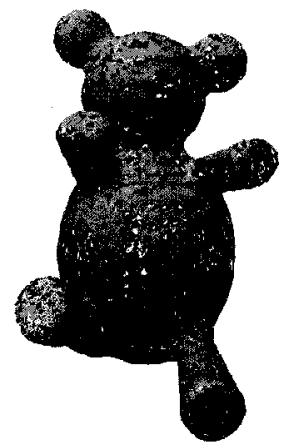

(a)

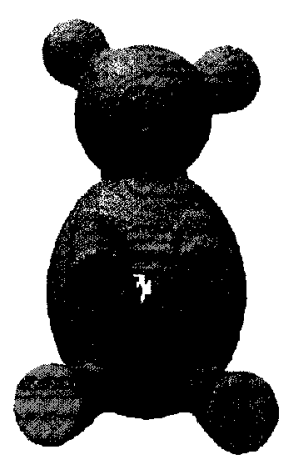

(b)

Figure 5.4: Models of teddy bears from the McGill 3D shape benchmark.

The second articulated model we experimented with is a subject of the CAESAR data base. The models of the CAESAR data base are available in three different postures. The three original models are shown in Figures 5.7(a) to (c). We manually changed the models to exclude the chair and fix large holes on the back for models (b) and (c). For model (b), the hands were detached from the legs, since our method cannot cope with changing topologies. Furthermore, we excluded one hand for each of the models to avoid symmetry problems during registration. The changed models are 


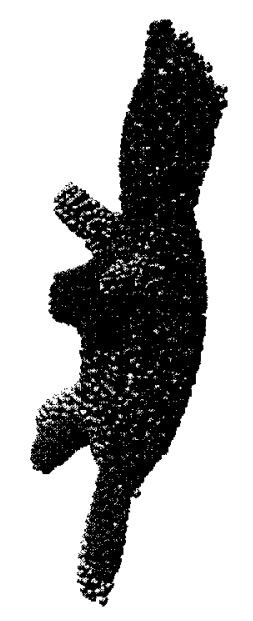

Figure 5.5: Optimal rigid alignment of canonical forms.

shown in Figures 5.8(a) to (c) and the modified models contain about 30,000 vertices. The sample sizes were chosen between 2,000 and 3,000 vertices.

Figure 5.9 shows the results of registering the models using the proposed algorithm. Figure 5.9(a) shows the registration of model (a) with model (c). We visualize the result by segmenting model (a), by assigning each segment a unique color, and by drawing vertices on the model (c) in same color as their corresponding vertices. We can see that the overall correspondence is correct, although some vertices of the head in (a) correspond to the left arm in (c). Figure 5.9(b) shows the registration of model (b) with model (c). We visualize the result by using the segmentation of model (c), by assigning each segment a unique color, and by drawing vertices on the model (b) in same color as their corresponding vertices. Note that the correspondence registered the left side of model (c) with the right side of model (b). This happens although we excluded one hand from consideration to avoid problems of this kind.

Since the emphasis of this chapter is on the fact that our algorithm is a theoretically more efficient approach than the approaches presented by Bronstein et al. [20] and Jain et al. [50], we developed a non-optimized experimental implementation. Hence, the running time of the experiments were just under twenty minutes for the largest of the data sets. Those running times could be improved by using multi-threading and by implementing some of the algorithms on the GPU as in Bronstein et al. [20]. 

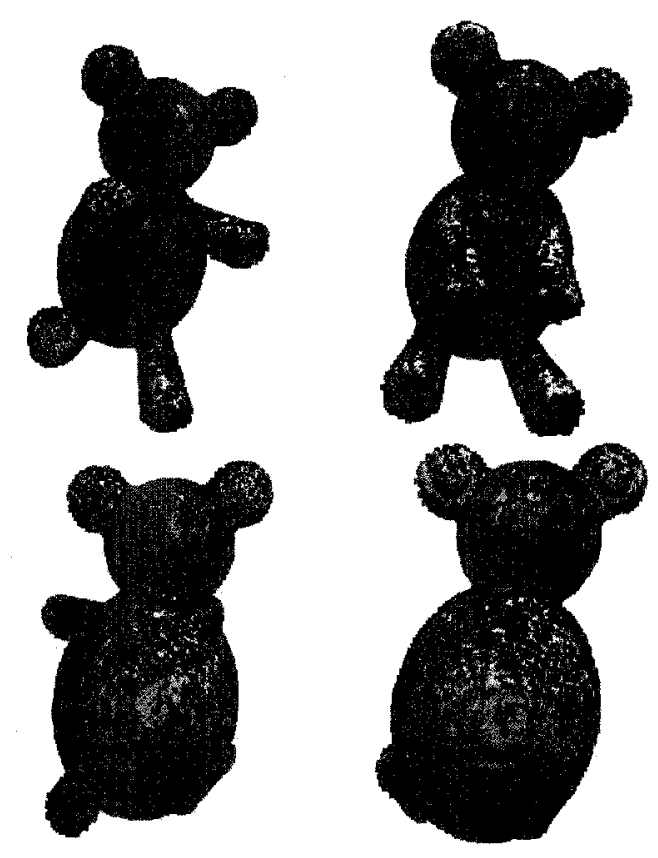

Figure 5.6: Fine correspondence computed by the algorithm.

\subsection{Conclusion}

This section summarizes the contribution of this work by comparing our algorithm to the algorithms proposed by Jain et al. [50] and to recent related work by Bronstein et al. [20]. Furthermore, we summarize limitations of our algorithm.

Our algorithm works well for non-symmetric surfaces which can be represented well in Euclidean spaces. The main advantage of our algorithm is that no prior knowledge about the objects being registered is required. The approach presented in this chapter is more time and space efficient than the approach by Jain et al. due to our coarse-to-fine strategy. While Jain et al.'s approach was a conceptual contribution, all of the experiments conducted were on small-scale models consisting of about 250 vertices. Our approach extends this concept and makes it applicable to real-life data sets with tens of thousands of vertices by overcoming the quadratic time and space complexity using a coarse-to-fine strategy.

The approach presented in this chapter can be viewed as a variation of the approach by Bronstein et al. Both approaches were developed independently at the same time. 


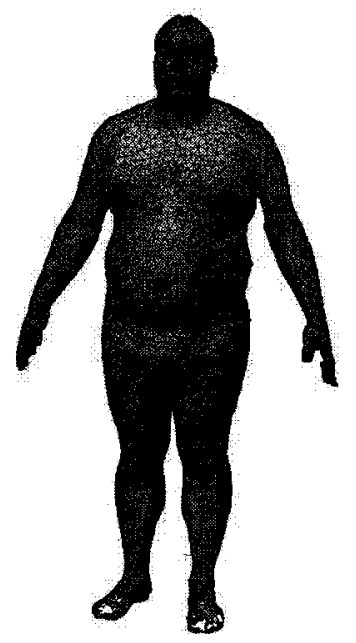

(a)

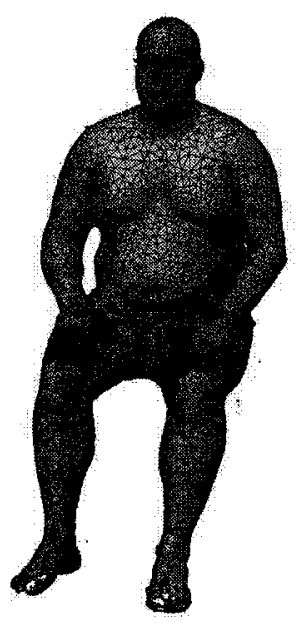

(b)

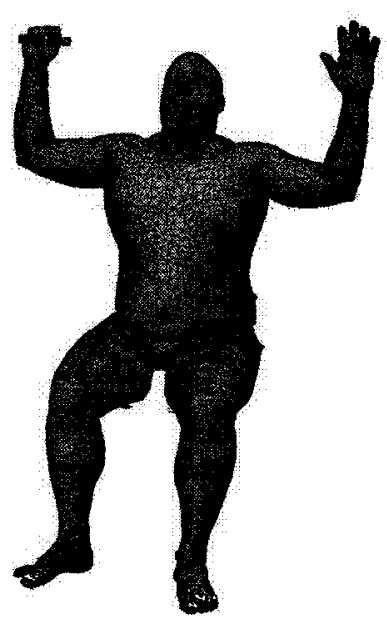

(c)

Figure 5.7: Models of CAESAR data base.

The main difference between the two approaches is the choice of the embedding space. While we embed to $\mathbb{R}^{k}$, Bronstein et al. use generalized multi-dimensional scaling to embed one manifold into another. While the approach of Bronstein et al. is more suitable to embed spaces that are not flat such as surfaces of human bodies, the approach assumes that one of the meshes is a complete mesh or a template mesh. Our approach does not assume this prior knowledge. Therefore, our approach is favorable for manifolds with nearly flat intrinsic geometry, where both models being registered contain significant amounts of missing data. Another limitation of Bronstein et al.'s approach is its high time complexity. To compute the canonical form, our approach recomputes the Euclidean distances $d_{i, j}(X)$ along with the gradient of $d_{i, j}(X)$ with respect to $X$ in each SMACOF iteration. The approach by Bronstein et al. needs to compute geodesic distances on the template mesh to replace $d_{i, j}(X)$. This means that in each SMACOF iteration, geodesic distances along with the gradient of the geodesic distances with respect to the positions of the points on the template surface need to be computed. Hence, while our algorithm takes $O\left(t n^{2}\right)$ time to compute the canonical form of $n^{\prime}$ sample points in $t$ iterations, the approach by Bronstein et al. takes $O\left(t n^{\prime 2} \log n^{\prime}\right)$ time. This makes our approach more efficient and therefore more suitable for large data sets. While our approach finds dense point-to-point 


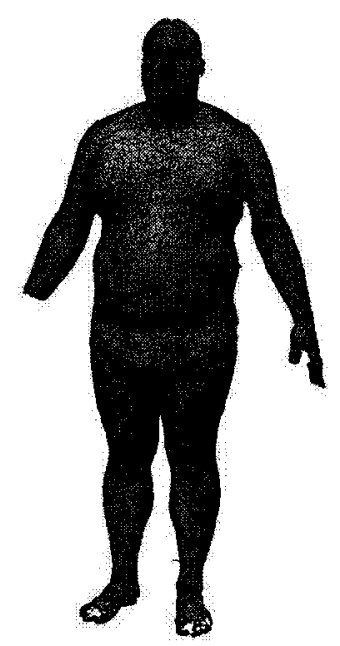

(a)

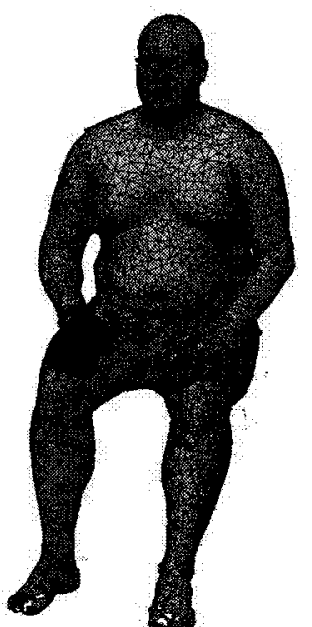

(b)

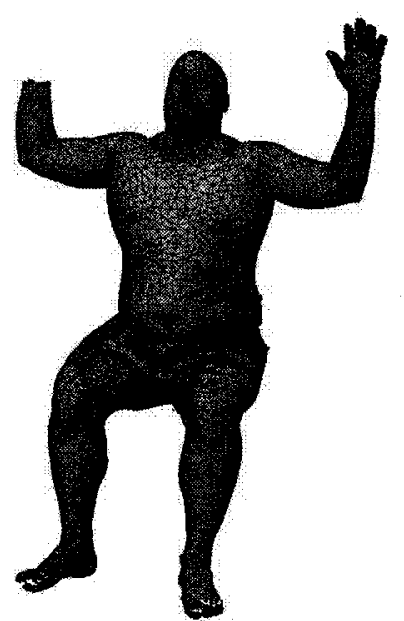

(c)

Figure 5.8: Modified models of CAESAR data base.

correspondences for data sets with tens of thousands of vertices, the approach by Bronstein et al. was only shown to compute correspondences between coarse sample sets containing about 3000 vertices.

Finally, we summarize some limitations of our approach that should be addressed in the future:

- Symmetric surfaces may be registered wrong by the coarse correspondence step of the algorithm. This limitation is addressed in the following chapters.

- Surfaces that cannot be represented well in Euclidean spaces cannot be registered reliably using this algorithm.

- Surfaces with large holes cannot be registered reliably using this algorithm, since large holes alter the global shape of the canonical embedding of the sample points. This is the reason we fixed the back parts of the sitting human models.

- Surfaces with many significant outliers cannot be registered reliably using this algorithm, because MDS is not robust with respect to outliers [28]. This means that outliers can alter the global shape of the canonical embedding of the sample points. 

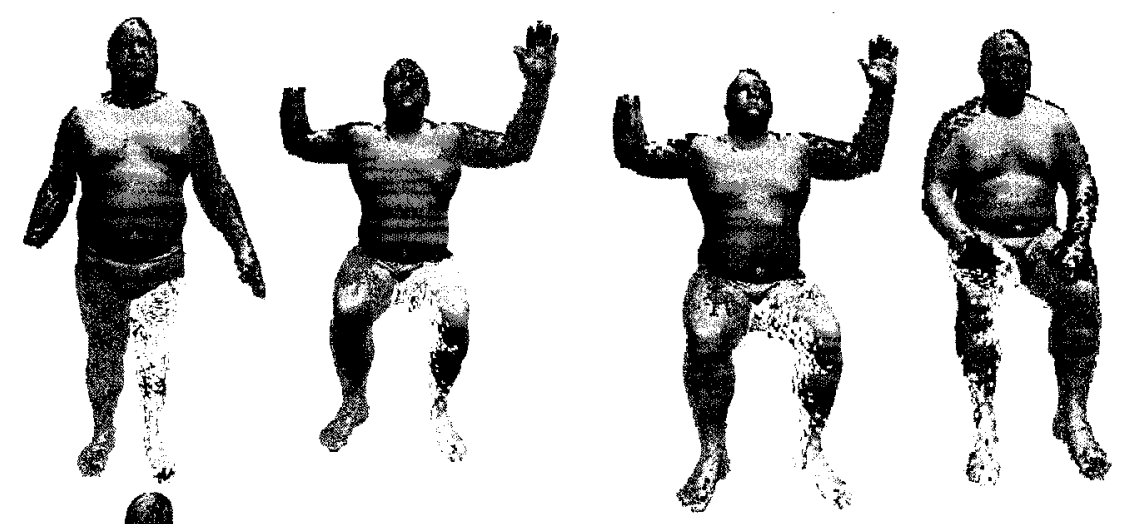

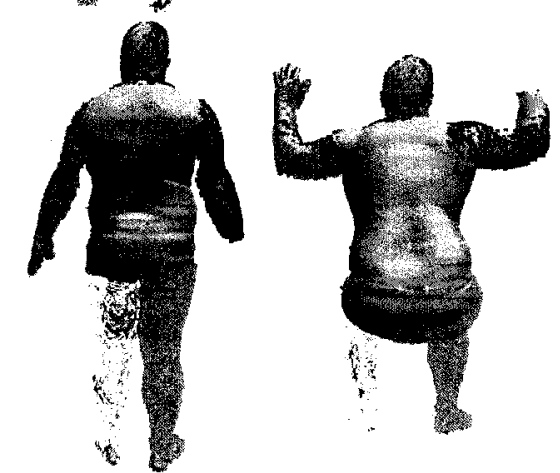

(a)

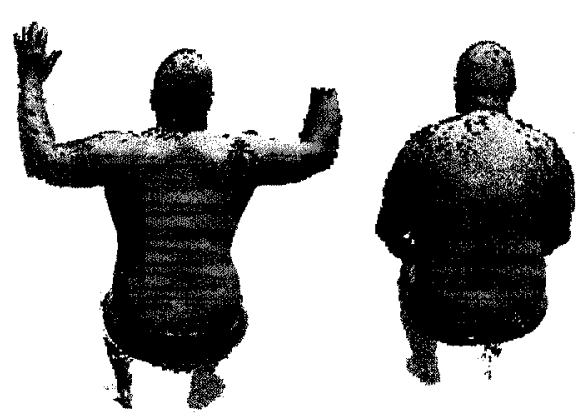

(b)

Figure 5.9: Fine correspondence computed by the algorithm. 


\title{
Chapter 6
}

\section{Morphing of Triangular Meshes in Shape Space}

\author{
I do not accept or desire in physics any other principles than in geometry \\ or abstract mathematics; because all the phenomena of nature are explained \\ thereby, and certain demonstrations concerning them can be given. \\ RENÉ DescaRTES (1596-1650)
}

We can use a deformation distance to overcome the symmetry problem encountered in the previous chapter. This chapter therefore considers morphing. The following chapter will use the results of this chapter to overcome the symmetry problem.

This chapter presents a novel approach to morph between two isometric poses of the same non-rigid object given as triangular meshes. We model the morphs as linear interpolations in a suitable shape space $\mathcal{S}$. For triangulated $3 D$ polygons, we prove that interpolating linearly in this shape space corresponds to the most isometric morph in $\mathbb{R}^{3}$. We then extend this shape space to arbitrary triangulations in $3 D$ using a heuristic approach and show the practical use of the approach using experiments. The newly presented approach solves the morphing problem without the need to solve a minimization problem. Furthermore, we discuss a modified shape space that is useful for isometric skeleton morphing. Finally, we present a method that applies the shape space to segment triangular meshes into near-rigid components. 


\subsection{Introduction}

We consider morphing in order to use the morphing techniques to overcome the symmetry problem encountered in the previous chapter. Interpolating smoothly between two given poses of the same non-rigid object, known as morphing, is interesting in its own right as it arises from many geometry processing problems. For example, morphing can compute an animation between given poses of a human or an animal because humans and animals deform approximately isometrically. Given two isometric poses of the same non-rigid object as triangular meshes $S^{(0)}$ and $S^{(1)}$ with known pointto-point correspondences, we aim to find a smooth isometric deformation between the poses.

Recently, Kilian et al. [55] showed that an isometric morph can be computed by finding the geodesic path in a shape space. A mesh that contains $n$ vertices is usually represented in shape space as a vector in $\mathbb{R}^{3 n}$ that contains all the vertex coordinates of the mesh. The mesh is positioned in a standard position to be translation and rotation invariant. That is, a shape is a vector of dimension $\mathbb{R}^{3 n}$ that contains all the geometrical information about the shape and that is invariant under location and rotation [54]. Computing geodesic paths in this space involves solving a large-scale non-linear optimization problem. Non-linear optimization problems are usually solved iteratively starting from an initial solution. This is computationally expensive. It is difficult to find the best solution because convergence problems may arise if the initial solution is far from the optimum. Finding a good initial solution is not straight forward. Kilian et al. overcome the inefficiency of the approach and the difficulty of finding a good initial solution by implementing two multi-resolution schemes. This is difficult to implement.

The objective of this chapter is to propose an alternative representation of the shape space. Instead of encoding the extrinsic geometry of the mesh in shape space as Kilian et al., we encode the intrinsic geometry of the mesh in shape space. This has the effect that a point on a geodesic path in shape space simply linearly interpolates between the endpoints of the geodesic path. Hence, we replace solving a large-scale non-linear optimization problem with linearly interpolating between two points. This 
is more efficient, conceptually easier, and easier to implement.

A deformation of a shape represented by a triangular mesh is isometric if and only if all triangle edge lengths are preserved during the deformation [55]. We call a morph $S^{(t)}, 0<t<1$ between two (possibly non-isometric) shapes $S^{(0)}$ and $S^{(1)}$ most isometric if it minimizes the sum of the absolute values of the differences between the corresponding edge lengths of two consecutive shapes summed over all shapes $S^{(t)}$, for $t$ in $[0,1]$. Note that in a similar definition, Kilian et al. [55] use the $L_{2}$ instead of the $L_{1}$ metric to measure most isometric morphs. In this chapter, we examine isometric deformations of general triangular manifold meshes in $3 D$ and of triangulated $3 D$ polygons, which are triangular meshes with no interior vertices. We introduce a new shape space $\mathcal{S}$ for triangulated $3 D$ polygons that has the property that interpolating linearly in shape space corresponds to the most isometric morph in $\mathbb{R}^{3}$. We then extend this shape space to arbitrary triangulations in $3 D$ using a heuristic approach. Furthermore, we discuss a modification of the shape space that is useful for isometric skeleton morphing. Finally, we apply the shape space to segment triangular meshes in to near-rigid components.

Related work is summarized in Section 2.2 .

\subsection{Theory of Shape Space for Triangulated $3 D$ Polygons}

This section introduces a novel shape space for triangulated $3 D$ polygons with the property that interpolating linearly in shape space corresponds to the most isometric morph in $\mathbb{R}^{3}$. The dimensionality of the shape space is linear in the number of vertices of the deformed polygon.

We start with two triangulated $3 D$ polygons $P^{(0)}$ and $P^{(1)}$ corresponding to two near-isometric poses of the same non-rigid object. Poses are considered near-isometric if the stretching between the poses is small. We assume that the point-to-point correspondence of the vertices $P^{(0)}$ and $P^{(1)}$ are known. Furthermore, we assume that both $P^{(0)}$ and $P^{(1)}$ share the same underlying mesh structure $M$. Hence, we know the 
mesh structure $M$ with two sets of ordered vertex coordinates $V^{(0)}$ and $V^{(1)}$ in $\mathbb{R}^{3}$, where $M$ is an outer-planar graph. We will show that we can represent $P^{(0)}$ and $P^{(1)}$ as points $p^{(0)}$ and $p^{(1)}$ in a shape space $\mathcal{S}$, such that each point $p^{(t)}$ that is a linear interpolation between $p^{(0)}$ and $p^{(1)}$ corresponds to a triangular mesh $P^{(t)}$ isometric to $P^{(0)}$ and $P^{(1)}$ in $\mathbb{R}^{3}$.

As we know the point-to-point correspondence of the vertices $P^{(0)}$ and $P^{(1)}$, we can find the best rigid alignment of the two shapes by solving an overdetermined linear system of equations and by modifying the solution to ensure a valid rotation matrix. To modify the solution to the overdetermined linear system, we compute a singular value decomposition of the solution matrix and replace the singular value matrix by the identity matrix [37].

Let $M$ consist of $n$ vertices. As $M$ is a triangulation of a $3 D$ polygon with $n$ vertices, $M$ has $2 n-3$ edges and $n-2$ triangles. We assign an arbitrary but fixed order on the vertices, edges, and faces of $M$. The shape space $\mathcal{S}$ is defined as follows. The first 3 coordinates of a point $p \in \mathcal{S}$ correspond to the coordinates of the first vertex $v$ in $M$. Coordinates 4 and 5 of $p$ correspond to the direction of the first edge of $M$ incident to $v$ in spherical coordinates. The next $2 n-3$ coordinates of $p$ are the lengths of the edges in $M$ in order. The final $2(n-2)$ coordinates of $p$ describe the outer normal directions of the triangles in $M$ in spherical coordinates, in order. An example of a shape space of a triangle is shown in Figure 6.1. Hence, the shape space $\mathcal{S}$ has dimension $5+2 n-3+2(n-2)=4 n-2=\Theta(n)$.

In the following, we prove that interpolating linearly between $P^{(0)}$ and $P^{(1)}$ in shape space yields the most isometric morph. To interpolate linearly in shape space, we interpolate the edge lengths by a simple linear interpolation. That is, $p_{k}^{(t)}=$ $t p_{k}^{(0)}+(1-t) p_{k}^{(1)}$, where $p_{k}^{(x)}$ is the $k$ th coordinate of $p^{(x)}$. The normal vectors are interpolated using geometric spherical linear interpolation (SLERP) [96]. That is, $p_{k}^{(t)}=\frac{\sin (1-t) \Theta}{\sin \Theta} p_{k}^{(0)}+\frac{\sin t \Theta}{\sin \Theta} p_{k}^{(1)}$, where $\Theta$ is the angle between the two directions that are interpolated.

Note that the relative rigid alignment of $P^{(0)}$ and $P^{(1)}$ in $\mathbb{R}^{3}$ has an influence on the linear interpolation. That is, the interpolating shape space point varies as the relative rigid alignment of $P^{(0)}$ and $P^{(1)}$ in $\mathbb{R}^{3}$ changes. The change occurs because the 

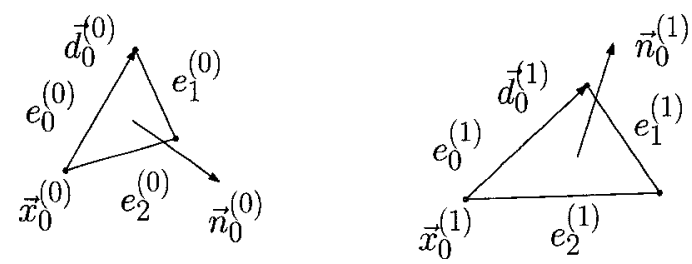

$$
p^{(0)}=\left(\begin{array}{c}
\vec{x}_{0}^{(0)} \\
\vec{d}_{0}^{(0)} \\
e_{0}^{(0)} \\
e_{1}^{(0)} \\
e_{2}^{(0)} \\
\vec{n}_{0}^{(0)}
\end{array}\right)
$$$$
p^{(1)}=\left(\begin{array}{c}
\vec{x}_{0}^{(1)} \\
\vec{d}_{0}^{(1)} \\
e_{0}^{(1)} \\
e_{1}^{(1)} \\
e_{2}^{(1)} \\
\vec{n}_{0}^{(1)}
\end{array}\right)
$$

Figure 6.1: The shape space $\mathcal{S}$ of a triangle.

angles between the normal vectors of $p^{(t)}$ change as a result of the rigid transformation. This is the reason we choose to find the best rigid alignment of $P^{(0)}$ and $P^{(1)}$ before transforming the polygons into $\mathcal{S}$.

To study interpolation in shape space, we make use of the dual graph $D(M)$ of $M$. The dual graph $D(M)$ has a node for each triangle of $M$. We denote the dual node corresponding to face $f$ of $M$ by $D(f)$. Two nodes of $D(M)$ are joined by an arc if the two corresponding triangles in $M$ share an edge. We denote the dual arc corresponding to an edge $e$ of $M$ by $D(e)$. Note that because $M$ meshes a $3 D$ polygon, it is an outer-planar triangular graph and so the dual graph of $M$ is a binary tree. An example of a mesh $M$ with its dual graph $D(M)$ is shown in Figure 6.2.

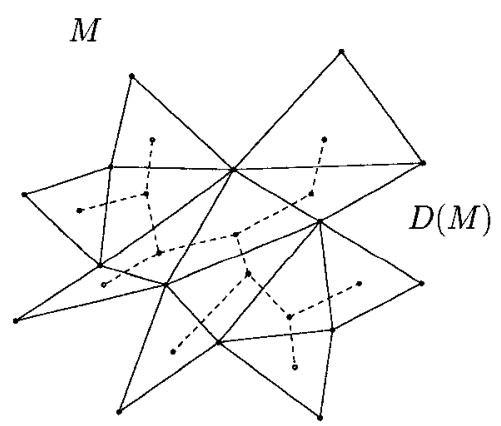

Figure 6.2: $A$ mesh $M$ with its dual graph $D(M)$. 
Theorem 6.2.1. Let $M$ be the underlying mesh structure of the triangulated $3 D$ polygons $P^{(0)}$ and $P^{(1)}$. The linear interpolation $p^{(t)}$ between $p^{(0)}$ and $p^{(1)}$ in shape space $\mathcal{S}$ for $0 \leq t \leq 1$ has the following properties:

1. There exists a unique mesh $P^{(t)} \in \mathbb{R}^{3}$ that corresponds to $p^{(t)} \in \mathcal{S}$ that has the underlying mesh structure $M$. The mesh $P^{(t)}$ is a valid triangular mesh. We can compute this mesh using a traversal of the binary tree $D(M)$ in $\Theta(n)$ time.

2. If $P^{(0)}$ and $P^{(1)}$ are isometric, then $P^{(t)}$ is isometric to $P^{(0)}$ and $P^{(1)}$. If $P^{(0)}$ and $P^{(1)}$ are not isometric, then each edge length of $P^{(t)}$ linearly interpolates between the corresponding edge lengths of $P^{(0)}$ and $P^{(1)}$.

3. The coordinates of the vertices of $P^{(t)}$ are a continuous function of $t$.

Proof. Part 1: To prove uniqueness, we start by noting that the first vertex $v$ of $P^{(t)}$ is uniquely determined by the first three coordinates of $p^{(t)}$. The direction of the first edge $e$ of $M$ incident to $v$ is uniquely determined by coordinates 4 and 5 of $p^{(t)}$, because each point on the unit sphere determines a unique direction in $\mathbb{R}^{3}$. The length of each edge of $P^{(t)}$ is uniquely determined by the following $2 n-3$ coordinates. Furthermore, the outer normal of each triangle is uniquely determined by the following $2(n-2)$ coordinates, because each point on the unit sphere determines a unique direction in $\mathbb{R}^{3}$. Hence, the edge $e$ is uniquely determined. For a triangle $f$ containing $e$, we now know the position of two vertices of $f$, the plane containing $f$, and the three lengths of the edges of $f$. Assuming that the normal vectors in shape space represent right-hand rule counterclockwise traversals of each triangle, this uniquely determines the position of the last vertex of $f$. We can now determine the coordinate of each vertex of $P^{(t)}$ uniquely by traversing $D(M)$. We start the traversal of $D(M)$ at $D(f)$. Recall that the coordinates of the vertices of triangle $f$ are known. Hence, when traversing an arc $D(e)$ incident to $D(f)$, we know the vertex coordinates of the shared edge between the two triangles corresponding to endpoints of $D(e)$. Denote the endpoint of $D(e)$ not corresponding to $f$ by $f^{\prime}$. For $f^{\prime}$, we now know the position of two vertices of $f^{\prime}$, the plane containing $f^{\prime}$, and the three lengths of the edges of $f^{\prime}$. Hence, we can compute the position of the last vertex of $f^{\prime}$. Because we now know the 
coordinates of all the vertices of $f^{\prime}$, we can traverse all of the arcs in $D(M)$ incident to $D\left(f^{\prime}\right)$. In this fashion, we can set all of the vertex coordinates of $P^{(t)}$ by traversing $D(M)$. Because $D(M)$ is a tree, it is cycle-free. Hence, the coordinates of each vertex of $P^{(t)}$ are set exactly once. Because the complexity of $D(M)$ is $\Theta(n)$, the algorithm terminates after $\Theta(n)$ steps.

It remains to prove that $P^{(t)}$ is a valid triangular mesh, that is, that the three edge lengths of each triangle of $P^{(t)}$ satisfy the triangle inequality. We assume that both input meshes were valid triangular meshes. Hence, for any triangle $t$ with edge lengths $a^{(0)}, b^{(0)}, c^{(0)}$ in $P^{(0)}$ and $a^{(1)}, b^{(1)}, c^{(1)}$ in $P^{(1)}$, the following inequalities hold:

$$
a^{(0)}+b^{(0)} \geq c^{(0)}, b^{(0)}+c^{(0)} \geq a^{(0)}, c^{(0)}+a^{(0)} \geq b^{(0)}
$$

and

$$
a^{(1)}+b^{(1)} \geq c^{(1)}, b^{(1)}+c^{(1)} \geq a^{(1)}, c^{(1)}+a^{(1)} \geq b^{(1)} .
$$

In $P^{(t)}, a^{(t)}=(1-t) a^{(0)}+t a^{(1)}, b^{(t)}=(1-t) b^{(0)}+t b^{(1)}, c^{(t)}=(1-t) c^{(0)}+t c^{(1)}$ due to the linear interpolation of the end positions. Hence, $a^{(t)}+b^{(t)}=(1-t) a^{(0)}+t a^{(1)}+$ $(1-t) b^{(0)}+t b^{(1)}=(1-t)\left(a^{(0)}+b^{(0)}\right)+t\left(a^{(1)}+b^{(1)}\right) \geq(1-t) c^{(0)}+t c^{(1)}$. Similarly, we can show that $b^{(t)}+c^{(t)} \geq a^{(t)}$ and $c^{(t)}+a^{(t)} \geq b^{(t)}$. Hence, $P^{(t)}$ is a valid triangular mesh.

Part 2: The edge lengths of $P^{(t)}$ are linear interpolations between the edge lengths of $P^{(0)}$ and $P^{(1)}$. Hence, the claim follows.

Part 3: When varying $t$ continuously, the point $p^{(t)} \in \mathcal{S}$ varies continuously. Hence, the coordinate of the lengths of all the edges vary continuously. Because a direction $[\sin v \cos u, \sin v \sin u, \cos v]^{T}$ varies continuously if $u$ and $v$ vary continuously, the normal directions vary continuously. Because all the vertex positions of the mesh $P^{(t)}$ are uniquely determined by continuous functions of those quantities, all vertex positions of $P^{(t)}$ vary continuously.

We do not need to solve minimization problems to find the shortest path in shape space as in Kilian et al. [55]. The only computation required to find an intermediate deformation pose is a graph traversal of $D(M)$.

Because $D(M)$ has complexity $\Theta(n)$, we can traverse $D(M)$ in $\Theta(n)$ time. Hence, we can compute intermediate deformation poses in $\Theta(n)$ time each. We denote this 
the polygon algorithm in the following. In the following, we show that the polygon algorithm computes the most isometric morph.

Corollary 1 . The polygon algorithm computes the most isometric morph between two triangulated $3 D$ polygons $P^{(0)}$ and $P^{(1)}$ with underlying mesh structure $M$.

Proof. The following argument shows that Part 2 of Theorem 6.2.1 implies that the most isometric morph is found. Consider an edge $e$ of $M$ and let $l(e)^{(t)}$ denote the length of $e$ in $P^{(t)}$. As all edge lengths are linearly interpolated during the morph, $e$ is linearly interpolated during the morph. Let the morph consist of $k+1$ poses $P^{(0)}, P^{\left(\frac{1}{k}\right)}, \ldots, P^{(1)}$. Recall that a morph is most isometric if it minimizes

$$
Q=\sum_{e \in E} \sum_{i=1}^{k}\left|l(e)^{\left(\frac{i}{k}\right)}-l(e)^{\left(\frac{i-1}{k}\right)}\right|,
$$

where $E$ is the edge set of $M$. It is

$$
\begin{aligned}
Q & \geq \sum_{e \in E} \sum_{i=1}^{k} l(e)^{\left(\frac{i}{k}\right)}-l(e)^{\left(\frac{i-1}{k}\right)} \\
& \geq \sum_{e \in E}\left(l(e)^{\left(\frac{1}{k}\right)}-l(e)^{(0)}+l(e)^{\left(\frac{2}{k}\right)}-l(e)^{\left(\frac{1}{k}\right)}+\ldots+l(e)^{(1)}-l(e)^{\left(\frac{k-1}{k}\right)}\right) \\
& \geq \sum_{e \in E} l(e)^{(1)}-l(e)^{(0)} .
\end{aligned}
$$

The linear interpolation achieves exactly $Q=\sum_{e \in E} l(e)^{(1)}-l(e)^{(0)}$, for it simply sums

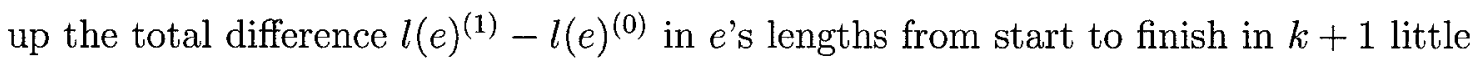
pieces. As linear interpolation achieves the lower bound on $Q$, the most isometric morph is found by the polygon algorithm.

\subsection{Theory of Shape Space for Skeleton Morphing}

A very similar shape space to the one presented in Section 6.2 can be used to isometrically morph between two topologically equivalent skeletons. Let a skeleton in $\mathbb{R}^{3}$ be a set of joints connected by links arranged in a tree-structure. That is, we can consider a skeleton to be a tree in $\mathbb{R}^{3}$ consisting of $n$ vertices and $n-1$ edges.

The shape space presented in Section 6.2 can be simplified to a shape space for skeletons in $\mathbb{R}^{3}$ with the property that interpolating linearly in shape space corresponds 
to the most isometric morph in $\mathbb{R}^{3}$. The dimensionality of the shape space is linear in the number of links of the skeleton.

We start with two skeletons $S^{(0)}$ and $S^{(1)}$ corresponding to two near-isometric poses. We assume that the point-to-point correspondence of $S^{(0)}$ and $S^{(1)}$ are known. Hence, we know the tree structure $T$ with two sets of ordered vertex coordinates $V^{(0)}$ and $V^{(1)}$ in $\mathbb{R}^{3}$. As before, we first find the best rigid alignment of the two skeletons.

The skeleton shape space $\mathcal{S}_{\mathcal{S}}$ is defined in a similar way as $\mathcal{S}$. We assign an arbitrary but fixed root to $T$ and traverse the edges of $T$ in a depth-first order. We assign an arbitrary order to the edges incident on each vertex of $T$. The first 3 coordinates of $s$ correspond to the coordinates of the root in $T$. The next $n-1$ coordinates of $s$ are the lengths of the edges in $T$ in depth-first order. The final $2(n-1)$ coordinates of $s$ describe the unit directions of the edges in spherical coordinates in depth-first order. All edges are oriented such that they point away from the root. Note that the shape space $\mathcal{S}$ has dimension $\Theta(n)$.

Interpolating linearly between points in $\mathcal{S}_{\mathcal{S}}$ is performed the same way as interpolating linearly between points in $\mathcal{S}$. Namely, edge lengths are interpolated linearly and unit directions are interpolated via SLERP. With a technique similar to the proof of Theorem 6.2.1, we can prove the following theorem. In the proof, we do not need to consider a dual graph, but we can simply traverse the tree $T$ in depth-first order to propagate the information.

Theorem 6.3.1. Let $T$ be the underlying tree structure of the skeletons $S^{(0)}$ and $S^{(1)}$. The linear interpolation $s^{(t)}$ between $s^{(0)}$ and $s^{(1)}$ in shape space $\mathcal{S}_{\mathcal{S}}$ for $0 \leq t \leq 1$ has the following properties:

1. The skeleton $S^{(t)} \in \mathbb{R}^{3}$ that corresponds to $s^{(t)} \in \mathcal{S}_{\mathcal{S}}$ is uniquely defined and has the underlying tree structure $T$. We can compute this tree using a depth-first traversal of the tree in $\Theta(n)$ time.

2. If $S^{(0)}$ and $S^{(1)}$ are isometric, then $S^{(t)}$ is isometric to $S^{(0)}$ and $S^{(1)}$. If $S^{(0)}$ and $S^{(1)}$ are not isometric, then each edge length of $S^{(t)}$ linearly interpolates between the corresponding edge lengths of $S^{(0)}$ and $S^{(1)}$.

3. The coordinates of the vertices of $S^{(t)}$ are a continuous function of $t$. 
This theorem allows us to morph isometrically between the skeletons of two shapes corresponding to two postures of the same articulated object in $\Theta(n)$ time. Figure 6.3 shows an example of such a morph.

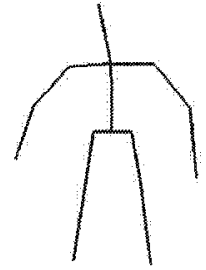

(a)

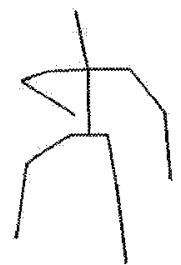

(b)

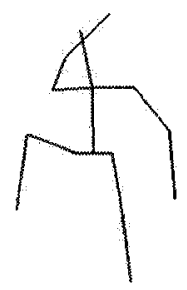

(c)

Figure 6.3: Example of most isometric morph between two skeleton poses. Input poses are shown in (a) and (c) and morph for $t=0.5$ is shown in (b).

In the remainder of this chapter, we will focus our attention on morphing between triangular meshes.

\subsection{Generalization to Triangular Meshes}

This section extends the shape space $\mathcal{S}$ from Section 6.2 to arbitrary connected triangular meshes. However, we can no longer guarantee the properties of Theorem 6.2.1, because the dual graph of the triangular mesh $M$ is no longer a tree.

Given two triangular meshes $S^{(0)}$ and $S^{(1)}$ corresponding to two near-isometric poses of the same non-rigid object with known point-to-point correspondence, we know one mesh structure $M$ with two sets of ordered vertex coordinates $V^{(0)}$ and $V^{(1)}$ in $\mathbb{R}^{3}$.

As before, we can use this information to find the best rigid alignment in $\mathbb{R}^{3}$. We do this before representing the shapes in a shape space. As outlined above, this alignment has a major influence on the result of the morph.

As before, we can represent $S^{(0)}$ and $S^{(1)}$ as points $s^{(0)}$ and $s^{(1)}$ in a shape space $\mathcal{S}$ using the same shape space points as in Section 6.2. Let $s^{(t)}$ be the linear interpolation of $s^{(0)}$ and $s^{(1)}$ in $\mathcal{S}$, where the linear interpolation is computed as outlined in Section 6.2. The existence of a mesh $S^{(t)} \in \mathbb{R}^{3}$ that has the underlying mesh structure $M$ and 
that corresponds to $s^{(t)}$ is no longer guaranteed. This can be seen using the example shown in Figure 6.4. Figure 6.4(a) and (b) show two isometric meshes $S^{(0)}$ and $S^{(1)}$. The dual graph $D(M)$ of the mesh structure $M$ is a simple cycle. Note that although the start and the end pose are isometric, we cannot find an intermediate pose that satisfies all of the interpolated normal vectors with SLERP and that is isometric to $S^{(0)}$ and $S^{(1)}$.

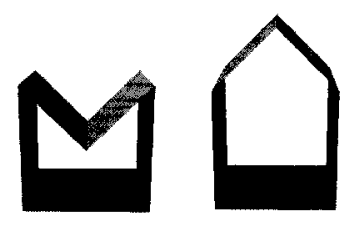

(a)

(b)

Figure 6.4: Example of isometric triangular meshes where intermediate poses interpolating all normals and edge lengths do not exist.

Let $M$ consist of $n$ vertices. As $M$ is a planar graph, $M$ has $\Theta(n)$ edges and $\Theta(n)$ triangles. The shape space $\mathcal{S}$ is defined using the same shape space points as in Section 6.2. The shape space $\mathcal{S}$ has dimension $\Theta(n)$. As before, we interpolate linearly in shape space by interpolating the edge lengths by a simple linear interpolation.

Observation 1. Given a triangular mesh $S^{(t)}$ with underlying mesh structure $M$, point $s^{(t)}$ in $\mathcal{S}$ is uniquely determined. However, the inverse operation, that is, computing a triangular mesh $S^{(t)}$ given a point $s^{(t)} \in \mathcal{S}$, is ill-defined.

This is illustrated in Figure 6.4.

To compute a unique triangular mesh $S^{(t)}$ given a point $s^{(t)} \in \mathcal{S}$ that linearly interpolates between $s^{(0)}$ and $s^{(1)}$, such that $S^{(t)}$ approximates the information given in $s^{(t)}$ well, we use the dual graph $D(M)$ of $M$. Unlike in Section $6.2, D(M)$ is not necessarily a tree. Our algorithm therefore operates on a minimum spanning tree $T(M)$ of $D(M)$. The tree $T(M)$ is computed by assigning a weight to each arc $e$ of $D(M)$. The weight of $e$ is equal to the difference in dihedral angle of the supporting planes of the two triangles of $M$ corresponding to the two endpoints of $e$. That is, we compute the dihedral angle between the two supporting planes of the two triangles of $M$ corresponding to the two endpoints of $e$ for the start pose $S^{(0)}$ and for the end 
pose $S^{(1)}$, respectively. The weight of $e$ is then set as the difference between those two dihedral angles, which corresponds to the change in dihedral angle during the deformation. The weight can therefore be seen as a measure of rigidity. The smaller the weight, the smaller the change in dihedral angle between the two triangles during the deformation, and the more rigidly the two triangles move with respect to each other. As $T(M)$ is a minimum spanning tree, $T(M)$ contains the arcs corresponding to the most rigid components of $M$.

Similar to Section 6.2 , we compute $S^{(t)}$ by traversing $T(M)$. However, unlike in Section 6.2 , setting the vertex coordinates of a vertex $v$ of $S^{(t)}$ using two paths from the root of $T(M)$ to two triangles containing $v$ can yield two different resulting coordinates for $v$. An example of this situation is given in Figure 6.5, where the coordinates of $v$ can be set by starting at $\operatorname{root}(T(M))$, and traversing the $\operatorname{arcs} e_{2}$ and $e_{3}$ of $T(M)$ or by traversing the $\operatorname{arcs} e_{1}, e_{4}$, and $e_{5}$ of $M$. We call the different coordinates computed for $v$ in $T(M)$ candidate coordinates of $v$. Our algorithm computes the coordinates of each vertex $v \in S^{(t)}$ as the average of all the candidate coordinates of $v$.

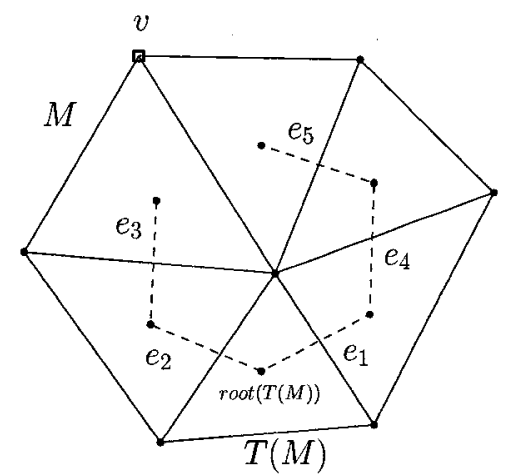

Figure 6.5: $A$ mesh $M$ with its dual minimum spanning tree $T(M)$.

Let us analyze the maximum number of candidate coordinates that can occur for a vertex in $S^{(t)}$. Let $e$ denote an edge of $M$ such that $D(e)$ is in $T(M)$. Let $v$ denote the vertex of $S^{(t)}$ opposite $e$ in the triangle corresponding to an endpoint of $D(e)$, such that the coordinates of $v$ are computed when traversing $D(e)$. This situation is illustrated in Figure 6.6. Let $d_{1}$ and $d_{2}$ denote the total number of candidate coordinates of the two endpoints of $e$. By traversing $D(e)$, we compute $d_{1} d_{2}$ candidate coordinates for $v$. 
We can therefore bound the number of candidate coordinates of $v$ computed using the path through $D(e)$ by $d_{1} d_{2}$. Note that the number of candidate coordinates for the two endpoints of the first edge is one. Furthermore, each vertex $v$ can be reached by at most $\operatorname{deg}(v)$ paths in $T(M)$, where $\operatorname{deg}(v)$ denotes the degree of vertex $v$ in $M$. As each path in $T(M)$ has length at most $m-1$, where $m=O(n)$ is the number of triangles of $M$, we can bound the total number of candidate coordinates in $S^{(t)}$ by $\sum_{v \in V} 2^{m-1} \operatorname{deg}(v)=2 n 2^{m-1}$, where $V$ is the vertex set of $M$.

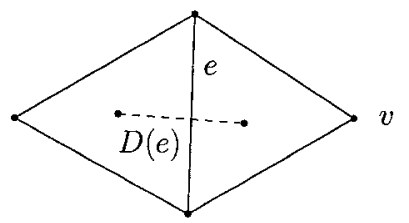

Figure 6.6: Illustration of how to bound the number of candidate coordinates of $v$ computed using the path through $D(e)$.

Our algorithm finds a triangular mesh $S^{(t)}$ corresponding to $s^{(t)}$ that is isometric to $S^{(0)}$ and $S^{(1)}$ if such a mesh exists, because all of the candidate coordinates are equal in this case and taking their average yields the desired result. If there is no isometric mesh corresponding to $s^{(t)}$, our algorithm finds a unique mesh that weighs all the evidence given by $T(M)$ equally. By choosing $T(M)$ as a minimum spanning tree based on weights representing rigidity, we allocate rigid parts of the model more emphasis than non-rigid parts. The reason for this is that in most near-isometric morphs, triangles close to non-rigid joints are deformed more than triangles in mainly rigid parts of the model. We conclude with the following.

Proposition 1. Let $S^{(0)}$ and $S^{(1)}$ denote two isometric connected triangular meshes and let $s^{(0)}$ and $s^{(1)}$ denote the corresponding shape space points, respectively. We can compute a unique triangular mesh $S^{(t)}$ representing the information given in the linear interpolation $s^{(t)}, 0 \leq t \leq 1$ of $s^{(0)}$ and $s^{(1)}$, in exponential time. We find a triangular mesh $S^{(t)}$ corresponding to $s^{(t)}$ that is isometric to $S^{(0)}$ and $S^{(1)}$ if such a mesh exists.

The algorithm can easily be extended to work for a non-connected triangular mesh $M$ by removing rigid transformations for each connected component of $M$ using local 
coordinate systems. We can then adapt the algorithm by finding the dual graph $D(M)$ and a minimum spanning tree $T(M)$ for each connected component of $M$. With this information, we can traverse the graph as described above. We denote this the exponential algorithm in the following.

\subsection{Efficient Algorithm to Deform Triangular Meshes}

As the exponential algorithm is limited for pragmatic reasons to triangular meshes with few vertices, this section describes a more computationally efficient algorithm to find the deformed poses.

To compute a unique triangular mesh $S^{(t)}$ given a point $s^{(t)} \in \mathcal{S}$ that linearly interpolates between $s^{(0)}$ and $s^{(1)}$, such that $S^{(t)}$ approximates the information given in $s^{(t)}$ well, we use the minimum spanning tree $T(M)$ of the dual graph $D(M)$ of $M$ as before. An approach that reduces the total number of candidate coordinates of each vertex of $S^{(t)}$ to $O(n)$ is to restrict to one the number of times each edge of $T(M)$ can be traversed. We traverse $T(M)$ in depth-first order. When an edge $D(e)$ is traversed, we add candidate coordinates to one vertex $v$ as shown in Figure 6.6. However, we only add at most a linear number of candidate coordinates to $v$ as described below. Denote by $v_{0}(e)$ and $v_{1}(e)$ the vertices of $e$, denote by $d_{1}$ and $d_{2}$ the number of candidate coordinates that were added to $v_{0}(e)$ and $v_{1}(e)$, respectively, during the traversal of $T(M)$ before traversing the edge $D(e)$, and let $v_{0}(e)$ be the vertex of $e$ that was updated more recently in the traversal of $T(M)$. Let the candidate coordinates of $v_{0}(e)$ $\left(v_{1}(e)\right.$, respectively) be given by $c_{1}^{1}, \ldots, c_{1}^{d_{1}}\left(c_{2}^{1}, \ldots, c_{2}^{d_{2}}\right.$, respectively) ordered from the least recently to the most recently added candidate coordinate. When traversing $D(e)$, we add $d_{2}$ candidate coordinates to $v$ by computing coordinates of $v$ based on the candidate pairs $\left(c_{1}^{d_{1}}, c_{2}^{1}\right), \ldots,\left(c_{1}^{d_{1}}, c_{2}^{d_{2}}\right)$.

This strategy computes $O(n)$ candidates per vertex of $S^{(t)}$, and hence a total of $O\left(n^{2}\right)$ candidates, thereby avoiding the computation of an exponential number of candidate coordinates. To find the final coordinate of a vertex $v$, we average all of the 
candidate coordinates of $v$. We denote this the averaging algorithm in the following.

The averaging approach was found to yield satisfactory results in all test cases. Just as in the algorithm by Kilian et al. [55], the results depend heavily on the initial rigid alignment of the shapes.

Furthermore, the results of the averaging algorithm depend on the order of the vertices and edges in $M$. Recall that the first vertex and its first incident edge are known. As we limit the number of times an edge of $T(M)$ can be traversed, not all of the information is propagated through the graph. Hence, the first vertex and the first edge influence the final result. We can eliminate this dependence at the cost of a higher running time by taking $O(n)$ shape spaces; one with each possible oriented edge as first edge. The running time of this algorithm is $O\left(n^{3}\right)$, which is too high for practical applications. We therefore can also use a smaller number of first edges that are found using Voronoi sampling [35]. If $O(\log n)$ samples are used, the running time of the algorithm becomes $O\left(n^{2} \log n\right)$ and the influence of the first edge is expected to be low. We do not include experiments obtained using these extensions of the approach as the averaging algorithm yields similar results as the less efficient algorithms in all of our experiments.

\subsection{Experimental Results}

This section presents experiments using the algorithms introduced in this chapter. We show exact morphs of triangulated $3 D$ polygons, morphs of models with few vertices computed using the exponential algorithm, and morphs of larger triangular meshes computed using the averaging algorithm.

OpenMP was used to improve the efficiency of the algorithms. To compute the minimum spanning tree $T(M)$, the boost graph library [97] was used.

\subsubsection{Deforming Triangulated $3 D$ Polygons}

We demonstrate the efficient polygon algorithm using the simple polygon shown in Figure 6.8. We deform the polygon shown in Figure 6.8 (a) to the polygon shown in 
Figure 6.8 (i). The morph is illustrated in Figures $6.8(\mathrm{~b})-(\mathrm{h})$. All of the intermediate poses are isometric to the start and end poses. The overlayed poses are shown in Figure 6.7. The running time of this example is less than 1 second.

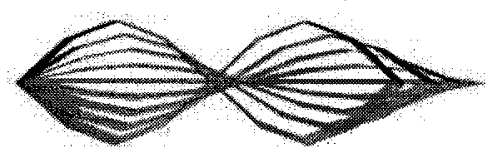

Figure 6.7: Isometric morph of a simple polygon. The start polygon is a $3 D$ polygon obtained by discretizing the curve $y=\sin (x)$ and by adding thickness to the curve along the $z$-direction. The end polygon is similarly obtained from $y=-\sin x$.

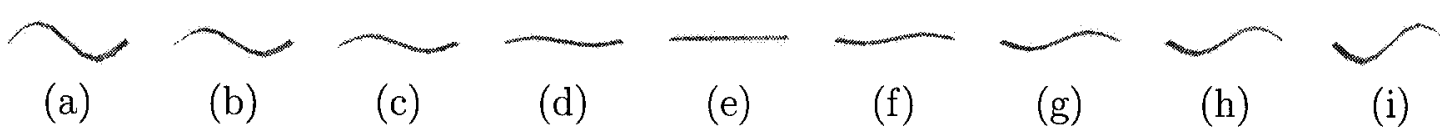

Figure 6.8: Isometric morph of a simple polygon from pose (a) to pose (i). Intermediate poses obtained using the polygon algorithm are given from left to right.

\subsubsection{Deforming General Triangular Meshes}

We present experimental results for the exponential algorithm and the averaging algorithm. First, we run the exponential algorithm on one model with few vertices to demonstrate the quality of the results. The model we use to test the approach is shown in Figure 6.9. We aim to smoothly and isometrically deform the pose shown in Figure 6.9(a) to the pose shown in Figure 6.9(i). As mentioned above, there is no isometric deformation between the poses that interpolates the triangle normals. The result of our algorithm is shown in Figures 6.9(b)-(h). Note that all triangle normals are interpolated and the symmetry of the model is preserved. Furthermore, all edge lengths with the exception of the edges of the four top faces are interpolated.

Second, we demonstrate the quality and efficiency of the averaging algorithm. The first experiment morphs between animated poses of the armadillo model. The models are chosen from the AIM@SHAPE repository ${ }^{1}$. The models contain 331904 triangles

\footnotetext{
${ }^{1}$ http://shapes.aimatshape.net/releases.php
} 


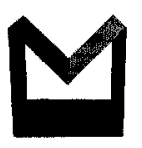

(a)

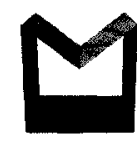

(b)

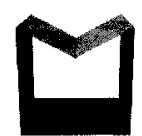

(c)

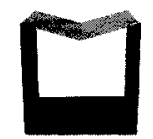

(d)

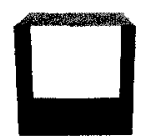

(e)

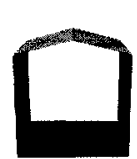

(f)

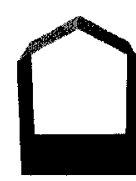

(g)

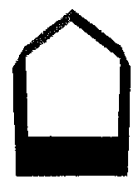

(h)

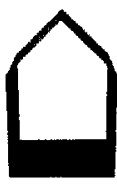

(i)

Figure 6.9: Most isometric morph of a cycle from pose (a) to pose (i). Intermediate poses obtained using the exponential algorithm are given from left to right.

and 165954 vertices. Testing our algorithms on these models emphasizes the practical use of our method. The results are shown in Figure 6.10. Note that the intermediate poses are visually pleasing. Although some small bumps appear on the right arm of the armadillo in Figure 6.10 (b) and on the left arm of the armadillo in Figure 6.10 (e), most details are preserved during the morph.

Figure 6.11 shows the morph between two poses of a model of a human being. The deformed pose of the model was found using the automatic technique by Baran and Popovic [10]. The models contain 10002 vertices. The two given meshes are shown in Figures 6.11(a) and (i). Intermediate poses using the averaging algorithm are shown in Figures 6.11(b) to (h). Note that although small bumps appear on the leg of the morphed surface, the morphs are intuitive.

Furthermore, we morph between heads from the CAESAR database [85]. The correspondence between the two head models was found using the approach by Xi et al. [112]. The models contain 11102 vertices. The two given meshes are shown in Figures 6.12(a) and (i). Intermediate poses obtained using the averaging algorithm are shown in Figure 6.12 (b) to (h). Note that the morphs are visually pleasing.

Finally, we morph between a few poses of the Alien model shown in Figure 6.13. The Alien model is chosen from the Princeton Shape Benchmark [95] and animated to obtain multiple postures with known correspondences using the automatic technique by Baran and Popovic [10]. All of the Alien models contain 429 vertices. The results are shown in Figure 6.13 and we can see that all of the morphs are visually pleasing.

For all of the experiments conducted, we measured the time efficiency to compute one intermediate pose at $t=0.5$. The running times range from under one second for the smaller models to about 30 minutes for the armadillo models. Note that the 


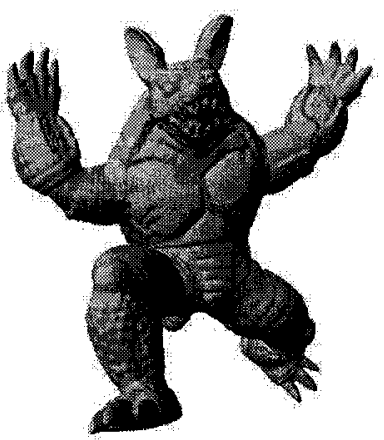

(a)

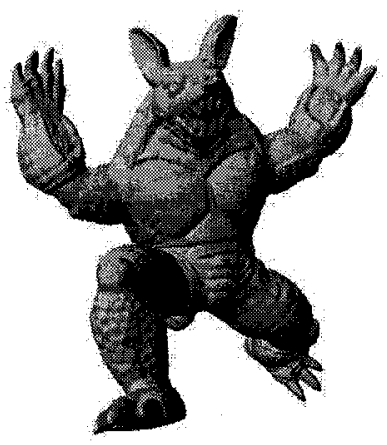

(d)

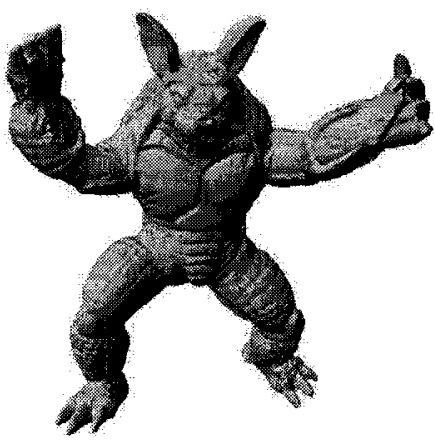

(b)

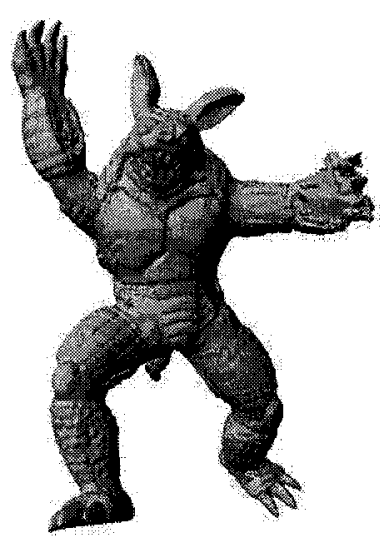

(e)

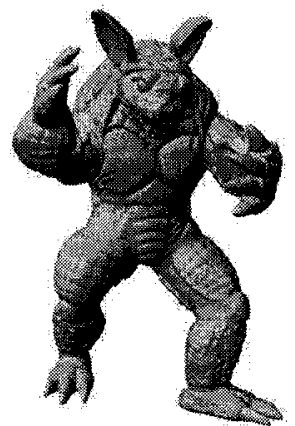

(c)

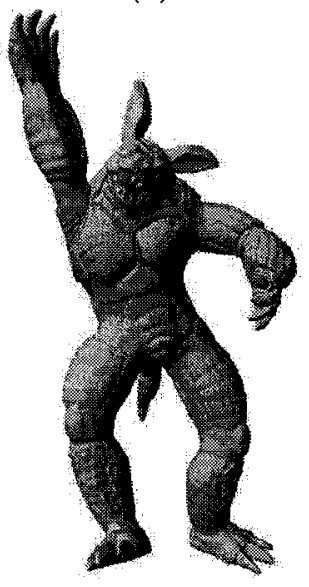

(f)

Figure 6.10: Most isometric morph of the armadillo model from pose (a) to pose (c) and from pose (d) to pose $(f)$. The intermediate poses obtained using the averaging algorithm for $t=0.5$ are given in figures (b) and (e).

developed implementation is non-optimized and experimental.

The running times might be improved by using a multi-resolution technique as follows. First, use progressive meshes [44] to obtain a low-resolution mesh containing $O(\sqrt{n})$ vertices. Progressive meshes are suitable for this task because the overall shape of an object is maintained. Second, compute the morph using this low-resolution mesh in $O(n)$ time. Third, add the vertices back one by one using vertex splits. Compute the coordinate of the newly added vertex $v$ by averaging the candidate coordinates computed using the $\operatorname{deg}(v)$ triangles containing $v$, where $\operatorname{deg}(v)$ denotes the degree of $v$. Adding $O(\sqrt{n})$ vertices in this fashion takes $O(n)$ time. Hence, the total time to 


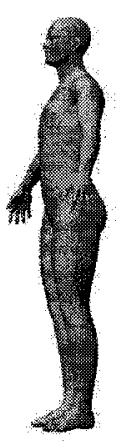

(a)

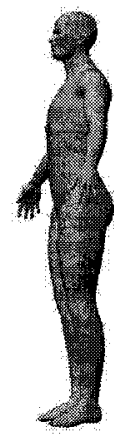

(b)

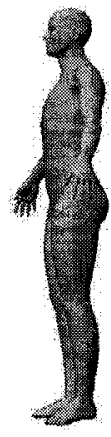

(c)

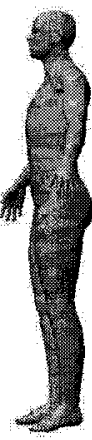

(d)

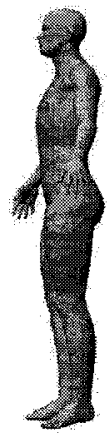

(e)

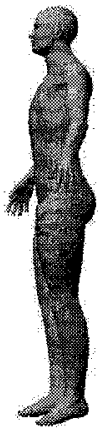

(f)

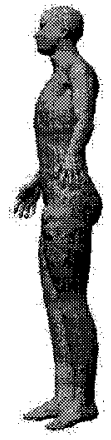

(g)

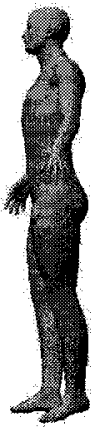

(h)

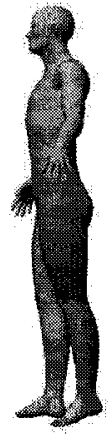

(i)

Figure 6.11: Most isometric morph of a human from pose (a) to pose (i). Intermediate poses obtained using the averaging algorithm are given from left to right.

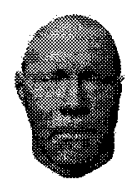

(a)

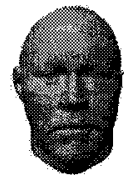

(b)

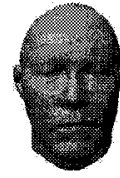

(c)

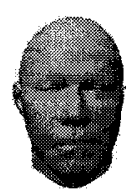

(d)

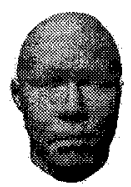

(e)

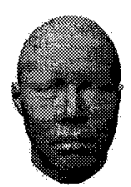

(f)

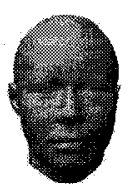

(g)

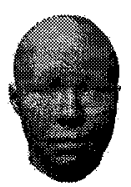

(h)

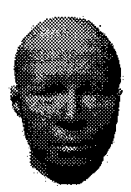

(i)

Figure 6.12: Most isometric morph of a head from pose (a) to pose (i). Intermediate poses obtained using the averaging algorithm are given from left to right.

compute a morph is $O(n)$ when this multi-resolution technique is used. Furthermore, the running times might be improved by implementing some of the algorithms on the GPU.

\subsection{Application to Segmentation Into Near-Rigid Components}

An extension of our newly developed shape space can be used to solve the problem of segmenting a deforming triangular mesh into near-rigid components based on $k$ given poses of the same non-rigid object. We model the segmentation problem as a clustering problem in dual space and find near-rigid segments with the property that segment boundaries are located at regions of large deformation. The presented approach is asymptotically faster than previous approaches that achieve the same 


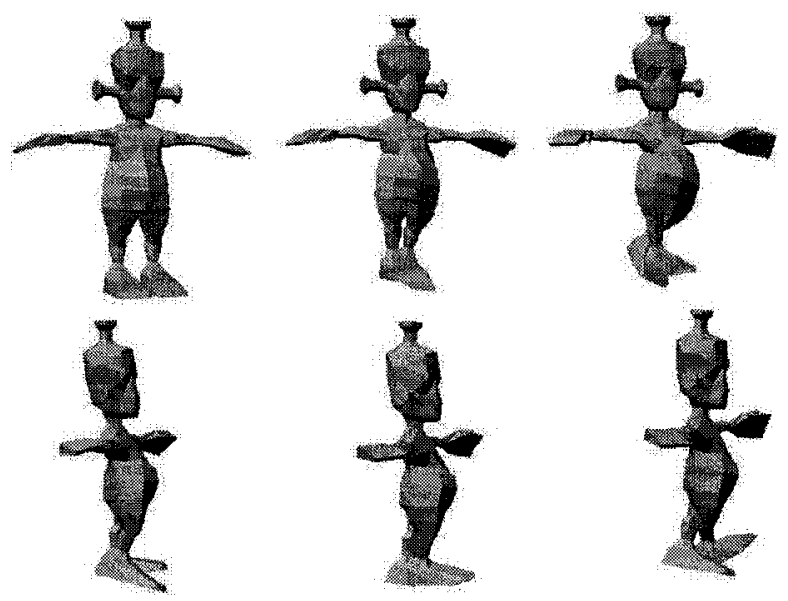

Figure 6.13: The two rows show start and end poses on the left and right, respectively. The intermediate poses shown in the middle columns are interpolations for $t=0.5$ obtained using the averaging algorithm.

property. The presented approach does not require any user-specified parameters.

The mesh segmentation problem is interesting in its own right. Mesh segmentation is an important tool in computer graphics. Different applications require mesh segmentations such as morphing, texture mapping, mesh simplification, and skeleton extraction. The type of mesh segmentation that is required depends strongly on the application. In the following, we focus on near-rigid mesh segmentations. For a recent survey on mesh segmentations in general, refer to Shamir [93]. Segmenting a mesh into near-rigid components based on a given set of deforming input meshes has various applications in geometry processing and computer graphics such as skeleton extraction [51] and skeleton-based morphing [51,68, 104].

Most previous methods segment one given static mesh [53], [70], [74]. Recently, several methods were proposed that consider segmenting a mesh based on a given set of deforming meshes with known point-to-point correspondence [52], [51], [88], [66]. Katz et al. [52] transform the given poses into a multi-dimensional space with the property that all of the poses are similar in this space. James and Twigg [51] consider the rotational sequences between corresponding triangles in different poses. Each rotational sequence is mapped to a point in a high-dimensional space and the near-rigid components are found using mean-shift clustering in this space. This clustering is used 
for skeleton extraction and animation. Although James and Twigg find near-rigid components, they do not find a segmentation of the mesh because some triangles in deforming regions do not belong to any cluster. Sattler et al. [88] pursue a similar approach. However, unlike James and Twigg, they do not analyze the motion of each triangle of the mesh, but the motion of each vertex of the mesh. Vertices with similar motions are clustered to obtain a segmentation. However, the experimental results show that a near-rigid segmentation is not always obtained. Lee et al. [66] propose an algorithm to find a near-rigid segmentation. The algorithm initially finds feature triangles on the mesh and uses those to grow clusters. The clusters are grown according to a distance metric between triangles based on a combination of geodesic distances and deformation distances. Although visually pleasing results are obtained, the algorithm is too slow for practical purposes since all pairwise distances between triangles are computed. Furthermore, the algorithm requires user-specified parameters.

We propose a novel method to segment a mesh into near-rigid components. Given $k$ poses of the same non-rigid object as triangular meshes $S^{(0)}, \ldots, S^{(k-1)}$ with known point-to-point correspondences, we aim to partition the mesh into near-rigid segments with the property that segment boundaries are located at regions of large deformation. Let $S^{(0)}, \ldots, S^{(k-1)}$ contain $n$ vertices each. We achieve this goal by modeling the segmentation problem as a clustering problem in dual space. Our algorithm runs in $O\left(k^{2} n+n \log n\right)$ time, which is a significant improvement over the algorithm by Lee et al. that takes $\omega\left(n^{2}\right)$ time. Furthermore, our algorithm does not require any user-specified parameters.

We start with $k$ poses $S^{(0)}, \ldots, S^{(k-1)}$ of the same non-rigid object given as triangular meshes. We assume that $S^{(0)}, \ldots, S^{(k-1)}$ share the same underlying mesh structure $M$. Hence, we know the mesh structure $M$ with $k$ sets of ordered vertex coordinates $V^{(0)}, \ldots, V^{(k)}$ in $\mathbb{R}^{3}$. Let $M$ contain $n$ triangles. To find a near-rigid segmentation of $M$, we make use of $D(M)$ and of a weighting scheme of edges in $D(M)$ similar to the previously introduced weighting scheme.

We assign a weight to each edge $e$ of $D(M)$. The weight of $e$ is equal to the maximum difference in dihedral angle of the supporting planes of the two triangles of $M$ corresponding to the two endpoints of $e$. That is, we compute the dihedral angle 
between the two supporting planes of the two triangles of $M$ corresponding to the two endpoints of $e$ for all the poses $S^{(0)}, \ldots S^{(k-1)}$. The weight of $e$ is then set as the maximum difference between any pair of dihedral angles. We use these weights to compute a minimum spanning tree $T(M)$ of $D(M)$.

Next, we find a clustering in dual space. The goal is to find a segmentation with the property that segment boundaries are located at regions of the largest deformation. Note that regions of largest deformation in $M$ correspond to edges with large weights in $D(M)$. Hence, we can find the sought segmentation by finding a farthest $d$-partition of $D(M)$. Recall from Chapter 4 that we can find this by cutting the $d-1$ longest edges of $T(M)$.

As the number $d$ of clusters is unknown in our case, we delete all the edges of $T(M)$ that have weights larger than a threshold $t_{1}$. This results in a farthest partition of $D(M)$. It remains to compute the threshold $t_{1}$. To compute $t_{1}$ in a fully automatic manner, we analyzed the distribution of the edge weights for a given set of input meshes. We found that the distribution of the edge weights resembles an exponential distribution. We use the edge weights to learn the underlying exponential distribution via maximum likelihood estimation. We then set $t_{1}$ to the third quartile of the learned distribution. This way we are expected to keep $75 \%$ of the edges in $T(M)$.

The segmentation corresponding to a farthest clustering of $D(M)$ may contain a large number of small segments. In many applications such as skeleton extraction, small segments are undesirable. We therefore merge the small segments. This consists of two subproblems: evaluating which segments are considered to be small, and merging small segments with neighboring segments. Assume that we are given a threshold $t_{2}$ such that any segment with less than $t_{2}$ elements is considered to be small and any segment with at least $t_{2}$ elements is not considered to be small. We will discuss later how to compute $t_{2}$ in a fully automatic way.

To merge small segments with neighboring segments, we find the edge in $T(M)$ with minimum weight that connects a small segment to a neighboring segment. Once the edge is found, we merge the two segments joined by the edge and repeat. The algorithm terminates once no segment is considered to be small. This algorithm maintains the property that segment boundaries are located at regions of large deformation because 
we merge along the least deforming edges that are available. More precisely, the resulting segmentation $S$ has the property that $S$ has the segment boundaries along edges of largest deformation among all segmentations with minimum segment size $t_{2}$. Note that the clustering is similar to the size-constrained farthest partition defined in Chapter 4. However, unlike in Chapter 4, the size of a cluster is not constrained to be below a threshold.

It remains to compute the threshold $t_{2}$. To compute $t_{2}$ in a fully automatic way, we analyzed the distribution of the segment sizes for a given set of input meshes. We found that the distribution of the segment sizes resembles a normal distribution. We use the known segment sizes to learn the underlying normal distribution $\left(\mu, \sigma^{2}\right)$ via maximum likelihood estimation. We then set $t_{2}$ to $\mu+3 * \sigma$. This way, $99.7 \%$ of the segments are expected to be considered small.

Figure 6.14 shows an example of the computed near-rigid segments of a set of five armadillo models and on a set of ten horse models. The horse models are created and used by Sumner et al. [99] and contain 16843 triangles. For the armadillo, note how the upper arm, lower arm, hands, and on the armadillo's left, the fingers, form near-rigid components. Segments on the back of the armadillo follow along the boundary of the shell. For the horse, the near-rigid segmentation captures well the skeletal structure of the animal.

\subsection{Conclusion}

We presented an approach to morph efficiently between isometric poses of triangular meshes in a novel shape space. The main advantage of this morphing method is that the most isometric morph is always found in linear time when triangulated $3 D$ polygons are considered. For general triangular meshes, the approach cannot be proven to find the optimal solution. However, this chapter presents an efficient heuristic approach to find a morph for general triangular meshes that does not depend on solving a non-linear optimization problem.

The presented experimental results demonstrate that the heuristic approach yields visually pleasing results. The approach is not invariant with respect to the order of 


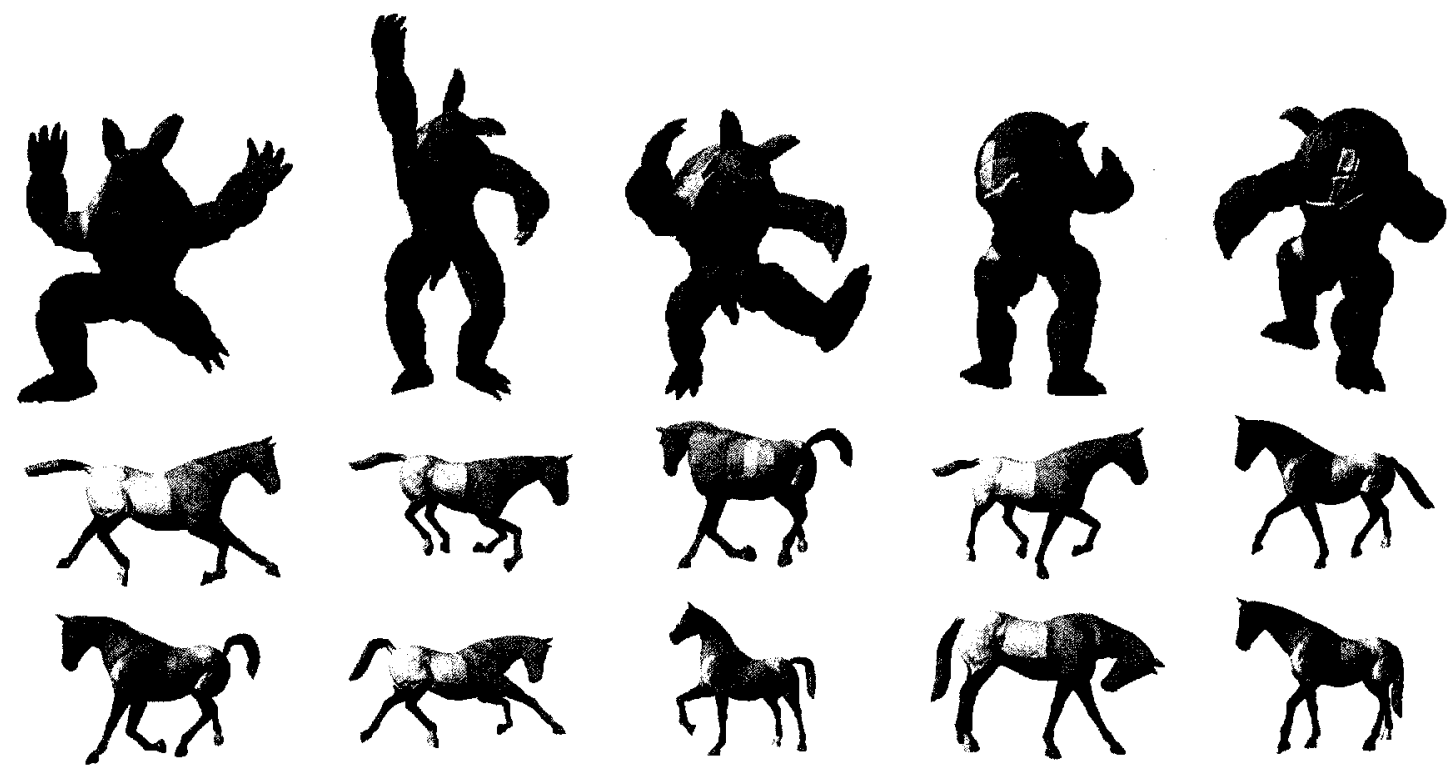

Figure 6.14: Near-rigid segmentation of the armadillo and the horse model.

the vertices of the mesh, but can be modified to have this property at the cost of a higher running time.

Furthermore, the newly developed shape space can also be applied to the problem of segmenting meshes into near-rigid parts.

An interesting direction for future work is to find an efficient way of morphing triangular meshes while guaranteeing that no self-intersections occur. For polygons in two dimensions, this problem was solved using an approach based on energy minimization [46].

We will use the newly developed shape space in the following chapter to overcome the symmetry problem when solving the correspondence problem. 


\section{Chapter 7}

\section{Combining Correspondence and Shape Space}

Animation offers a medium of story telling and visual entertainment which can bring pleasure and information to people of all ages everywhere in the world.

WALT DisNEY (1901-1966)

Chapter 5 proposes an algorithm to find dense point-to-point correspondences between two deformed possibly incomplete triangular meshes corresponding to different postures of the same non-rigid object in a fully automatic way. The approach models the deformations of an object as isometries and solves the correspondence problem by mapping the intrinsic geometries of the surfaces into a low-dimensional Euclidean space via multi-dimensional scaling. Recall that one problem arising during the rigid registration is the erroneous alignment of shapes with symmetric canonical forms. We overcome this problem by combining the algorithm presented in Chapter 5 with the shape space introduced in Chapter 6. This combination allows us to compute the correspondence that yields the most isometric morph. 


\subsection{Introduction}

As in Chapter 5, we consider the problem of finding dense point-to-point correspondences between two deformed surfaces $S^{(0)}$ and $S^{(1)}$ corresponding to different postures of the same non-rigid object. The aim is to use a deformation cost to overcome the symmetry problem encountered in Chapter 5.

Recently, two methods were proposed to find the point-to-point correspondences in a fully automatic way by minimizing a deformation cost $[45,116]$. These methods do not assume prior knowledge of markers or template shapes. However, both methods rely heavily on non-intuitive user-specified parameters. For a more thorough review of the two methods, refer to Section 2.2 .

We propose a new method to solve the correspondence problem in a fully automatic way that does not require any user-specified parameters. We find candidate correspondences using multi-dimensional scaling as in Chapter 5 . We then find the best alignment by choosing the candidate that has the shortest deformation distance. The deformation distance is measured in the shape space introduced in Chapter 6.

\subsection{Overview of the Algorithm}

This section gives an overview of the proposed algorithm. The approach starts by finding a set of candidate correspondences. The correct correspondence is found as the candidate correspondence that minimizes a deformation energy.

The candidate correspondences are found using multi-dimensional scaling as in Chapter 5. However, the approach presented in Chapter 5 has the drawback that near-symmetric shapes are registered erroneously. The reason is that the wrong candidate correspondence is accepted as the correct solution to the correspondence problem. Section 7.3 reviews how to efficiently find candidate correspondences.

To find the correct candidate correspondence, we evaluate a deformation cost for each candidate correspondence. The candidate correspondence with the smallest deformation cost is chosen as the final correspondence. To evaluate the deformation cost, we compute a deformation distance between $S^{(0)}$ and $S^{(1)}$ in the shape space 
introduced in Chapter 6. Section 7.4 outlines how to compute the deformation cost for a given candidate correspondence.

\subsection{Finding Candidate Correspondences Via Multi- Dimensional Scaling}

This section describes how we find candidate correspondences. First, we find coarse correspondences using the algorithm introduced in Section 5.3.1. Recall that this results in an assignment function $a(i)$ that assigns exactly one point $X_{a(i)}^{(1)}$ to every point $X_{i}^{(0)}$, where $X_{i}^{(r)}$ is the $i$-th vertex in the $r$-th canonical form for $r=0,1$.

To eliminate erroneous matchings that assign a point $X_{a(i)}^{(1)}$ to a point $X_{i}^{(0)}$, we employ a different approach than in Chapter 5 . We wish to only accept correspondences that form a graph $G^{(0)}$ on $S^{(0)}$ that is approximately isometric to a graph $G^{(1)}$ on $S^{(1)}$. We can find these correspondences using a kernel extraction method as proposed by Leordeanu and Hebert [67] and used by Huang et al. [45]. The approach proceeds as follows. We first compute a consistency matrix $C$ with entries

$$
c_{i, j}=\min \left(\frac{\delta_{i, j}^{(0)}}{\delta_{a(i), a(j)}^{(1)}}, \frac{\delta_{a(i), a(j)}^{(1)}}{\delta_{i, j}^{(0)}}\right) .
$$

Note that $0 \leq c_{i, j} \leq 1$. The matrix $C$ measures if two pairs of correspondences are consistent. They are consistent if and only if the geodesic distances $\delta_{i, j}^{(0)}$ on $S^{(0)}$ and $\delta_{a(i), a(j)}^{(1)}$ on $S^{(1)}$ are almost identical. In case of consistency, $c_{i, j}$ is close to 1 . To allow for small non-isometric deformations, we do not use $C$ to extract the valid correspondences, but define a matrix $M$ to find the kernel [45]. The entries of $M$ are

$$
m_{i, j}=\left\{\begin{array}{l}
\left(\frac{c_{i, j}-c_{0}}{1-c_{0}}\right)^{2} \text { if } c_{i, j}>c_{0}, \\
0 \text { otherwise }
\end{array}\right.
$$

where $c_{0}$ is a threshold. The threshold $c_{0}$ describes how much non-isometric deformation is acceptable. Unlike Huang et al. [45], we do not set the threshold empirically. Instead, to compute $c_{0}$ in a fully automatic manner, we analyzed the distribution of 
the entries of the matrix $C$ over all $2^{k}$ candidate correspondences. We found that the distribution resembles part of a Gaussian distribution. Note that for the different candidate alignments, the distributions of the entries of the matrix $C$ differ significantly. However, after summing all of the $2^{k}$ matrix entries, the distribution resembles a Gaussian in all of the cases we encountered. The distribution for one of the Alien models discussed in Section 7.5 is shown in Figure 7.1. The figure shows a histogram of the values $c_{i, j}$. We use the $2^{k}$ matrices $C$ (one matrix per candidate correspondence) to learn the underlying normal distribution $\left(\mu, \sigma^{2}\right)$ via maximum likelihood estimation. We then set $c_{0}$ to $\mu-3 * \sigma$. This way, $99.7 \%$ of the correspondences are expected to be accepted.
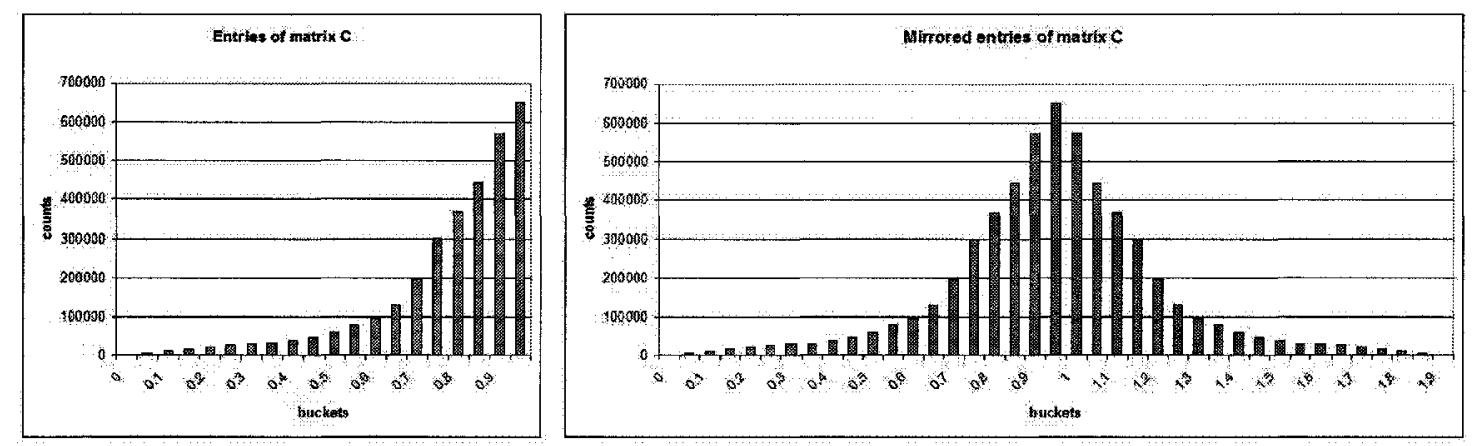

Figure 7.1: The distribution of the entries of the matrix $C$ over all $2^{k}$ candidate correspondences for one of the Alien models. The left side shows the distribution. The right side shows the Gaussian obtained by mirroring the values along $x=1$.

Finally, to compute the fine correspondence, all vertices of $S^{(r)} \backslash P^{(r)}$ are projected to the embedding space. As in Chapter 5, the approach finds the correspondence of the projected vertices by evaluating an approximating thin-plate spline mapping the embedding $X^{(0)}$ to $X^{(1)}$. The vertices of $S^{(r)} \backslash P^{(r)}$ are then registered using a nearest neighbor search. We only accept correspondences that are consistent with the sample correspondences according to the learned parameter $c_{0}$.

The running time of computing all $2^{k}$ candidate correspondences is

$$
O\left(n^{\prime} n \log n+n^{\prime 2} t+2^{k}\left(\left(n^{\prime}+k\right)^{3}+n(t k+\sqrt{n})\right)\right)
$$

where $n=\max \left(n^{(0)}, n^{(1)}\right), n^{\prime}=\max \left(n^{(0)}, n^{\prime(1)}\right), k$ is the dimension of the embedding space, and $t$ is the maximum number of iterations used to compute the embedding of 
the points. Since $k=3$ and since on average $n^{\prime}$ and $t$ are much smaller than $n$, the average running time of the algorithm is $O(n \sqrt{n})$. As discussed in Chapter 5 , this can be improved to $O\left(n k^{O(1)} \log n\right)$ by using a faster data structure to find the nearest neighbor in $\mathbb{R}^{k}[27,76]$.

The following section outlines how we choose the best candidate correspondence.

\subsection{Choosing a Candidate Correspondence}

Recall that we assume that $S^{(0)}$ and $S^{(1)}$ are near-isometric. Hence, we wish to accept the correspondence that deforms $S^{(0)}$ to $S^{(1)}$ in the most isometric way. To solve this problem, we need to compute a common mesh structure $M$ such that embedding $M$ into $\mathbb{R}^{3}$ with $V^{(0)}$ as vertices approximates $S^{(0)}$ and such that embedding $M$ into $\mathbb{R}^{3}$ with $V^{(1)}$ as vertices approximates $S^{(1)}$. Once $M$ is known, we need to evaluate a cost function such that minimizing the cost function over all candidate alignments yields the correspondence that deforms $S^{(0)}$ to $S^{(1)}$ in the most isometric way.

\subsubsection{Computing a Common Mesh $M$}

Given a candidate correspondence, we know two sets of ordered vertex coordinates: a set of vertex coordinates $V^{(0)}$ on $S^{(0)}$ and a set of vertex coordinates $V^{(1)}$ on $S^{(1)}$. Note that for $r=0,1, V^{(r)}$ is a subset of the vertices of $S^{(r)}$ because some vertices have no correspondence. Furthermore, we know the underlying mesh structures $M^{(0)}$ and $M^{(1)}$. Note that $M^{(0)}$ and $M^{(1)}$ do not have the same topology. Furthermore, $M^{(0)}$ and $M^{(1)}$ may be incomplete or even disconnected.

We compute two mesh structures $M^{\prime(r)}$ starting from $M^{(r)}$. The goal for $M^{\prime(r)}$ is to only contain vertices that are in the set $V^{(r)}$. We compute $M^{\prime(r)}$ from $M^{(r)}$ using half-edge collapses. We collapse all the half-edges until only vertices in $V^{(r)}$ remain. We always collapse the half-edge that results in the least change in volume. Lindstrom and Turk [71] explain how to find this half-edge. This results in two mesh structures on $V^{(r)}: M^{\prime(0)}$ and $M^{\prime(1)}$. Both $M^{\prime(0)}$ and $M^{\prime(1)}$ have the property that embedding them into $\mathbb{R}^{3}$ with $V^{(0)}$ as vertices approximates $S^{(0)}$ and that embedding them into 
$\mathbb{R}^{3}$ with $V^{(1)}$ as vertices approximates $S^{(1)}$.

\subsubsection{Evaluating a Cost Function}

Recall that $M$ denotes a mesh structure such that embedding $M$ into $\mathbb{R}^{3}$ with $V^{(0)}$ as vertices approximates $S^{(0)}$ and such that embedding $M$ into $\mathbb{R}^{3}$ with $V^{(1)}$ as vertices approximates $S^{(1)}$. Given $M, V^{(0)}$, and $V^{(1)}$, we can evaluate how isometric the two deformed shapes are by computing the energy

$$
E(M)=\sum_{(i, j) \in E}\left(d\left(v_{i}^{(0)}, v_{j}^{(0)}\right)-d\left(v_{i}^{(1)}, v_{j}^{(1)}\right)\right)^{2},
$$

where $E$ is the edge set of $M, v_{i}^{(r)}$ is the $i$-th vertex in the ordered set $V^{(r)}$ for $r=0,1$, and $d(p, q)$ denotes the Euclidean distance between $p$ and $q$. The energy $E(M)$ measures how isometric the shapes are because a deformation of a shape represented by a triangular mesh is isometric if and only if all triangle edge lengths are preserved during the deformation [55].

The energy $E(M)$ is a deformation distance in the shape space $\mathcal{S}$ introduced in Chapter 6. Kilian et al. [55] note that we can find the true point-to-point correspondences between two deformed triangular meshes over a set of possible candidate correspondence alignments by computing the distance in $\mathcal{S}$ for each alignment. The correct alignment is found as the alignment with minimum distance. This observation holds because the correct correspondence is the shortest geodesic path in shape space $\mathcal{S}$. Note that the shape space used by Kilian et al. simply encodes the extrinsic geometry of the deformed shapes. We use the shape space from Chapter 6 because this shape space encodes the intrinsic geometry of the deformed mesh. Hence, a geodesic path in our shape space is simply a straight line. Our shape space is therefore more suitable to measure the deformation distance than the shape space proposed by Kilian et al.

To obtain a symmetric deformation energy, we evaluate the energy twice and choose the cost as the maximum of the two results. That is, the deformation cost for a candidate correspondence is computed as

$$
E_{D}=\max \left(E\left(M^{\prime(0)}\right), E\left(M^{\prime(1)}\right)\right) .
$$




\subsection{Experimental Results}

This section presents experiments using the algorithm presented in this chapter. OpenMP was used to improve the efficiency of the algorithms. To minimize the energy when computing the canonical form, a quasi-Newton method is used. The quasi-Newton method used is the limited-memory Broyden-Fletcher-Goldfarb-Shanno scheme [73].

We first show some results of the correspondence algorithm. We then show how the computed correspondence can be applied to morphing and to finding near-rigid components. These two applications are discussed in detail in Chapter 6.

\subsubsection{Correspondence Results}

We first describe an experiment that evaluates the quality of our approach. The same experiment was conducted in Chapter 5. This allows us to compare the two approaches.

To conduct the experiment, we chose a model of an alien from the Princeton Shape Benchmark [95], subdivided the model to obtain a high resolution mesh, and animated the model to obtain multiple postures with known correspondences using the automatic technique by Baran and Popović [10]. Figure 7.2 illustrates the experiment. The three postures of the alien used to conduct the experiment consist of 6858 vertices. We use 2500 sample vertices to compute the canonical forms. The figure shows the correspondence found by the algorithm. We found the correspondences between posture (a) and postures (b),(c), and (d) in this experiment. Each vertex is assigned a color in posture (a). The corresponding points in postures (b),(c), and (d) are then displayed using the same color in row four of Figure 7.2. Note that some vertices do not obtain a color in postures (b),(c), and (d). The reason is that no correspondence was found for those vertices. We can see that a visually pleasing correspondence is found for all postures. Our implementation takes about 35 minutes to find each of the correspondences.

We compare the correspondences found by our algorithm to the ground truth by computing the geodesic distances between the correspondence found by the algorithm 
and the true correspondence for each vertex. We measure the error in correspondence as the number of edges along the shortest path between the correspondence found by the algorithm and the true correspondence. Since the algorithm rejects erroneous matchings automatically, some points do not obtain a correspondence. We do not assign an error to rejected correspondences. When registering posture (a) and posture (b), 5764 correspondences are rejected as erroneous. When registering posture (a) and posture (c), 4710 correspondences are rejected as erroneous. When registering posture (a) and posture (d), 4930 correspondences are rejected as erroneous. A histogram of the error encountered is shown in Figure 7.3. The histogram shows three different data sets: the set of errors when corresponding posture (a) to posture (b) is shown in light grey, the set of errors when corresponding posture (a) to posture (c) is shown in dark grey, and the set of errors when corresponding posture (a) to posture (d) is shown in white. Nearly all of the correspondences found by our algorithm between posture (a) and postures (c) and (d) are accurate within a distance of two edge lengths. This shows that the presented approach yields correspondences of high quality. In case of corresponding postures (a) and (b), the best candidate correspondence is found. The large correspondence error is a result of non-isometric models.

Note that unlike in the approach in Chapter 5 , no symmetry alignment problems occur. However, our approach accepts fewer correspondences.

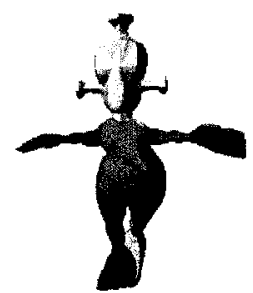

(a)

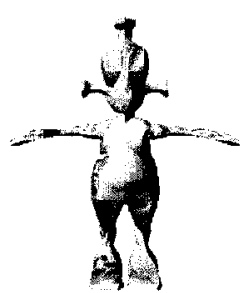

(b)

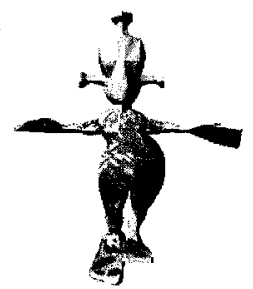

(c)

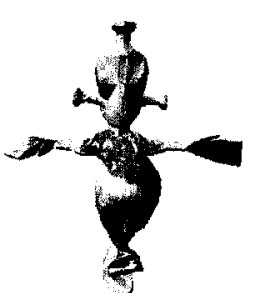

(d)

Figure 7.2: Color-coded correspondence for models of alien in postures (a) to (d) with known ground truth.

Second, we evaluated the proposed approach using two models with large non-rigid deformation. The models are shown in Figure 7.4. The cat models contain 7207 vertices and were created and used by Sumner et al. [99]. We use 2000 samples to 


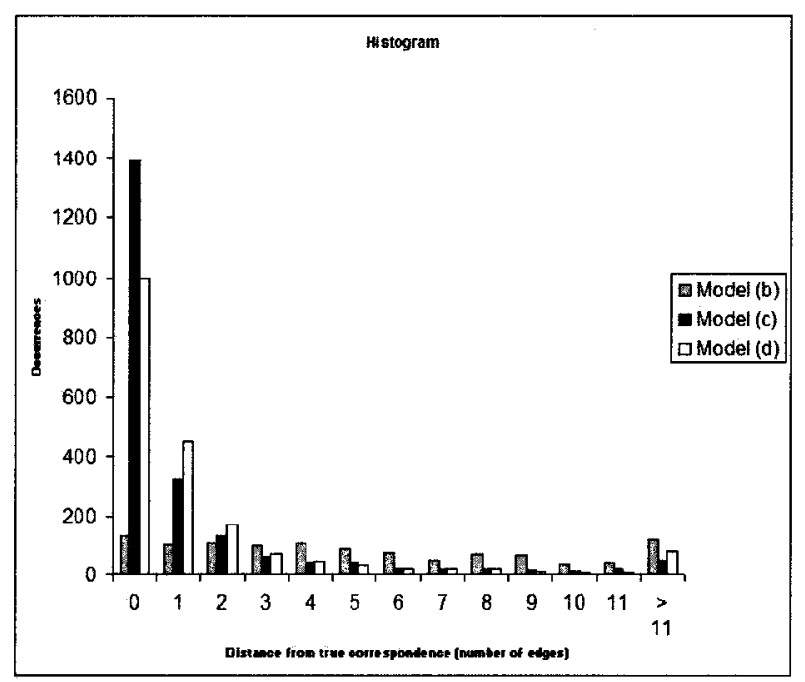

Figure 7.3: Histogram of the errors made by the correspondence algorithm.

compute the canonical forms. The gorilla models contain between 2028 and 2046 vertices and were created by Bronstein et al. [20]. We use 1000 samples to compute the canonical forms. Note that the correct correspondence is found for all of the shown poses. No symmetric alignment problems occur. This shows that the proposed approach is suitable for models with large deformation. Our implementation computes each of the correspondences in less than 10 minutes.
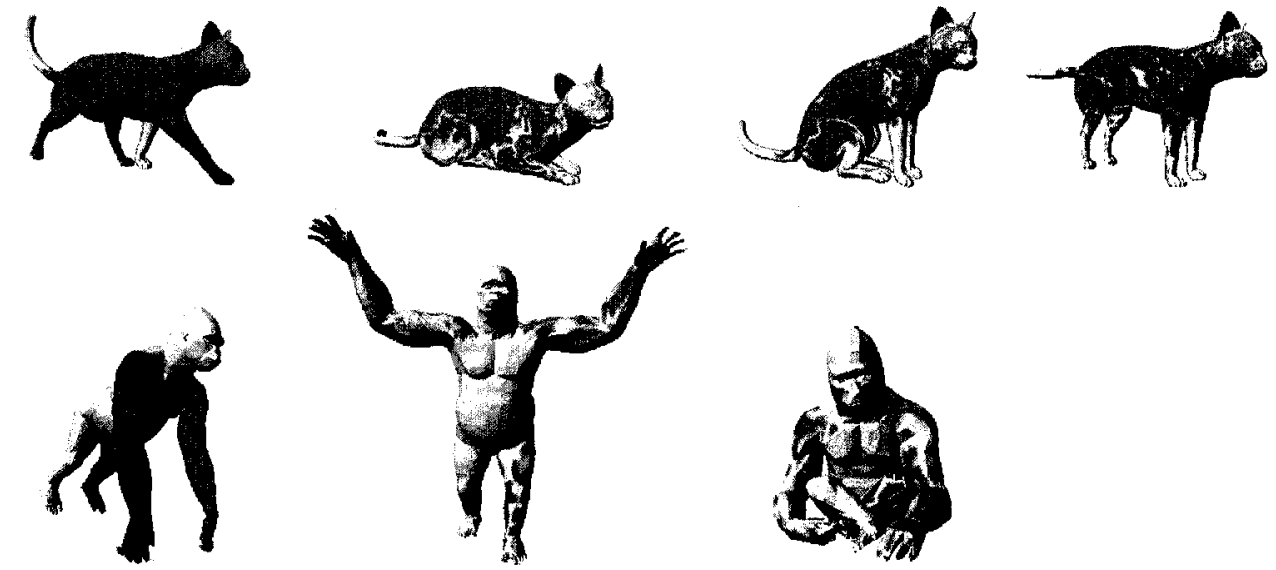

Figure 7.4: Color-coded correspondence for cat and gorilla models with large non-rigid deformation. 
Third, we evaluated the performance of the proposed approach for models with incomplete underlying meshes. The models are shown in Figure 7.5. The human model is a model from the CAESAR database [85]. We manually changed the models to exclude the chair and fix large holes on the back for model (b). Furthermore, for model (b), the hands were detached from the legs, since our method cannot cope with changing topologies. The modified models contain 10993 and 11476 vertices. We use 2000 samples to compute the canonical forms. The model of the bear is from the McGill 3D shape benchmark [117]. The models contain 21338 and 25658 vertices. Both models contain many small holes. We use 2050 samples to compute the canonical forms. Note that the correct correspondence is found for all of the shown poses. No symmetric alignment problems occur. This shows that the proposed approach is suitable for incomplete models with small holes. For the bear, our implementation computes the correspondences in about 80 minutes.

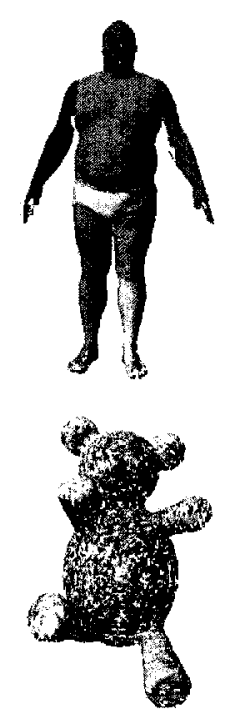

(a)
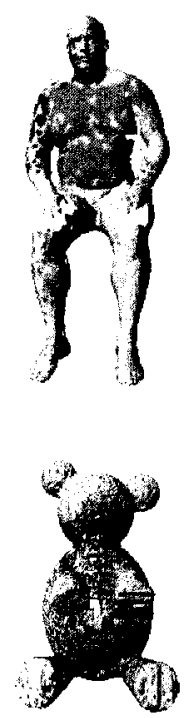

(b)

Figure 7.5: Color-coded correspondence for incomplete models of a human and a bear.

The proposed approach has limitations. One limitation we encountered in our experiments is due to the assumption that models deform approximately isometrically. If the models deform non-isometrically, the incorrect correspondence is found. An example of this is shown in Figure 7.6, where the human model is deformed into the 
third pose present in the CAESAR database. The torso of the human does not deform isometrically. Because of this, the wrong candidate correspondence is chosen and the front of the human in the standing pose is incorrectly matched to the back of the human in the sitting pose.
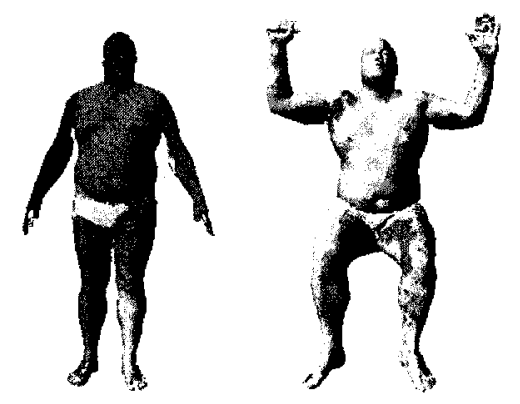

Figure 7.6: Color-coded correspondence for models of a human that do not deform isometrically. The wrong correspondence is found.

A further example of this limitation is shown in Figure 7.7. The models were created by Bronstein et al. [20]. Poses (e) and (f) are registered correctly. However, the remaining poses register incorrectly. Note that none of the incorrect poses is simply a symmetric misalignment. Instead, parts of the object are registered correctly and other parts of the object are registered incorrectly. For instance, in pose (b) the arms are registered correctly while the legs are registered incorrectly. Note that in those cases, none of the 8 candidate correspondences we compute is correct. This problem does not arise because we choose the incorrect candidate correspondence, but because none of the candidate correspondences is correct. This problem occurs although the embedding error is small when computing the canonical forms. This shows that the models are not isometric.

\subsubsection{Application to Morphing}

An application of the correspondence problem is the problem of morphing smoothly between two poses with known correspondence as discussed in Chapter 6. This section applies the proposed algorithm to the morphing problem. To morph, we first need to find a complete corresponding mesh. Note that the proposed algorithm only 


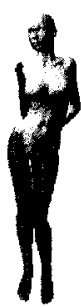

(a)

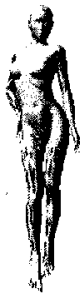

(b)

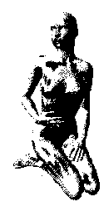

(c)

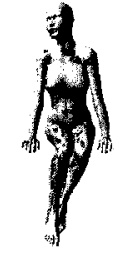

(d)

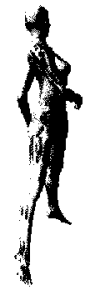

(e)

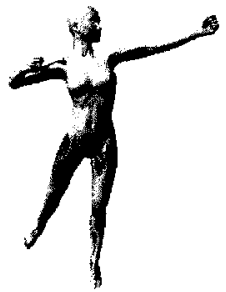

(f)

Figure 7.7: Color-coded correspondence for models of a dancer that does not deform isometrically.

computes point-to-point correspondences for a subset of the vertices. The reason is that erroneous correspondences are rejected using the kernel technique described above.

To compute two complete meshes in correspondence from one of the reduced meshes $M^{\prime(r)}$ for $r=0,1$, we use the mean-value geometry encoding introduced by Kraevoy and Sheffer [62]. With the mean-value encoding, we can find a deformation of $M^{(r)}$ that interpolates the points with known correspondence in $M^{((r+1) \bmod 2)}$. To compute this deformation, an energy needs to be minimized. We minimize this energy using numerical derivatives as in Kraevoy and Sheffer. We make the deformed mesh as isometric as possible to $M^{(r)}$ by minimizing the isometric energy introduced by Kilian et al. [55]. We slightly modify the energy to ensure that the deformed mesh interpolates the points with known correspondence in $M^{((r+1) \bmod 2)}$. An example of this application is shown in Figure 7.8. We start by computing the correspondence of the two models shown in grey in Figures 7.8(a) and (c). The reconstructed mesh is shown in blue in Figure 7.8(c). Note that the blue mesh is noisy and locally flipped at some places due to slight errors in correspondence. However, the mesh captures the overall shape of the model well.

Once the correspondence is known, we can morph between the grey mesh in Figure 7.8(a) and the blue mesh in Figure 7.8(c). As the deformed mesh is noisy, we do not morph using the shape space in Chapter 6. Instead, we morph using the technique by Kilian et al. [55]. Figure 7.8(b) shows the morph between the models shown in Figure 7.8(a) and (c). 


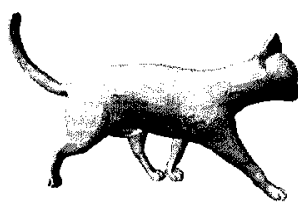

(a)

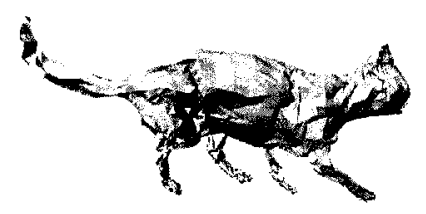

(b)

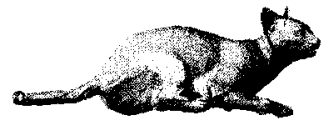

(c)

Figure 7.8: Application to morphing. (a): Original model. (c) Original model (grey) and correspondence (blue). (b) Morph between the models (a) and (c).

\subsubsection{Application to Finding Near-Rigid Components}

A second application of the correspondence problem is the problem of segmenting a mesh into near-rigid components based on a set of deformed input meshes as outlined in Section 6.7. This section applies the proposed algorithm to the problem of segmenting a mesh into near-rigid components. The complete meshes in correspondence used in this section are computed the same way as in the previous section.

Figure 7.9 shows the near-rigid segmentation obtained for the models shown in the top row of Figure 7.4. Note that the overall skeletal structure is captured. However, the face of the cat is over-segmented due to incorrect correspondences.

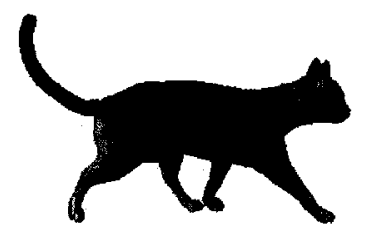

Figure 7.9: Near-rigid segmentation of the cat model.

\subsection{Conclusion}

This section summarizes the contribution of this work by comparing our algorithm to the recent related work by Zhang et al. [116] and Huang et al. [45]. Furthermore, we summarize limitations of our algorithm.

We find the correct correspondence by minimizing a deformation cost. This is similar to the recent approaches by Zhang et al. and Huang et al. Like the algorithms 
by Zhang et al. and Huang et al., our algorithm works well for isometric surfaces. No prior knowledge about the objects being registered is required. The algorithm by Huang et al. outperforms our algorithm in terms of running time. However, the algorithm by Huang et al. is not guaranteed to converge to the optimally best solution. The running time of Zhang et al.'s algorithm is comparable to the running time of our algorithm.

The main advantage of this work compared to the work by Zhang et al. and Huang et al. is the presentation of a parameter free method that solves the correspondence problem. This is an important contribution because the large number of non-intuitive user-specified parameters required by the algorithms by Zhang et al. and Huang et al. renders their algorithms impractical.

Finally, we summarize some limitations of our approach that should be addressed in the future:

- Surfaces that are not isometric cannot be registered reliably using this algorithm. We discuss examples of this limitation in Section 7.5.

- Surfaces with large holes cannot be registered reliably using this algorithm, since large holes alter the global shape of the canonical embedding of the sample points. This is the reason we fixed the back parts of the sitting human models.

- Surfaces that cannot be represented well in Euclidean spaces cannot be registered reliably using this algorithm. Although some of our models such as the gorilla have large embedding error, we never encountered this problem in our experiments. This problem is intrinsic to approaches that use canonical forms in $\mathbb{R}^{k}$ and can be avoided by computing canonical forms on template shapes [20].

- Surfaces with many significant outliers cannot be registered reliably using this algorithm, because MDS is not robust with respect to outliers [28]. This means that outliers can alter the global shape of the canonical embedding of the sample points. We never encountered this problem in our experiments. 


\section{Chapter 8}

\section{Conclusion}

Those who have handled sciences have either been men of experiment or men of dogmas. The men of experiment are like the ant; they only collect and use; the reasoners resemble spiders who make cobwebs out of their own substance. But the bee takes a middle course; it gathers material from the flowers of the garden and of the field, but transforms and digests it by a power of its own. Not unlike this is the true business of philosophy[.] FRANCIS BACON (1561-1626)

This chapter briefly summarizes the main contributions of this thesis and gives ideas for future work.

\section{Summary}

Using the techniques presented in this thesis, we can compute the point-to-point correspondence between two possibly incomplete triangular meshes $S^{(0)}$ and $S^{(1)}$ in a fully automatic way. The approach presented in this thesis works well if $S^{(0)}$ and $S^{(1)}$ are approximately isometric, have approximately Euclidean intrinsic geometry, and do not contain large holes or many outliers. The approach is based purely on the surfaces and does not depend on template meshes, marker positions, or user-specified parameters. 
The approach presented in this thesis only works for triangular manifolds. The only reason for this restriction is the approach used for computing geodesic distances. The correspondence framework can be extended to work for any surface representation that allows to evenly sample vertices on the surface and to compute all pairwise geodesic distances along the surface.

Currently, the approach does not work well for non-isometric surfaces. However, the general framework presented in this thesis can be extended to other classes of shapes if we can find suitable dissimilarities that can be used to compute near-rigid canonical forms.

We showed in Chapter 7 that the approach may fail for surfaces that have nonEuclidean intrinsic geometry. This limitation can be avoided by computing canonical forms in spherical space [28, Chapter 4.6] or on template shapes [20].

There are many directions for future research. We outline a few open problems below:

Open Problem 1: The approach presented here cannot register non-isometric surfaces in a fully automatic way. To our knowledge, there is no approach that can register non-isometric surfaces reliably without the use of markers, template shapes, or user-specified parameters. Is it possible to associate other classes of shapes with suitable dissimilarities that yield near-rigid canonical forms?

Open Problem 2: To our knowledge, all of the algorithms that automatically find point-to-point correspondences without using markers or template shapes fail for incomplete surfaces with large holes. This problem is especially challenging due to large portions of missing information.

Open Problem 3: To our knowledge, all of the algorithms that automatically find point-to-point correspondences without using markers or template shapes fail for noisy surfaces. 


\section{Bibliography}

[1] Marc Alexa. Recent advances in mesh morphing. Computer Graphics Forum, 21(2):173-196, 2002.

[2] Brett Allen, Brian Curless, and Zoran Popović. The space of human body shapes: reconstruction and parameterization from range scans. ACM Transactions on Graphics, 22(3):587-594, 2003. Proceedings of SIGGRAPH.

[3] Helmut Alt and Leonidas J. Guibas. Discrete Geometric Shapes: Matching, Interpolation, and Approximation. In: Jörg-Rüdiger Sack and Jorge Urrutia (Editors). The handbook of computational geometry, pages 121-153. Elsevier Science, 2000.

[4] Dragomir Anguelov, Praveen Srinivasan, Daphne Koller, Sebastian Thrun, Hoi-Cheung Pang, and James Davis. The correlated correspondence algorithm for unsupervised registration of nonrigid surfaces. In Neural Information Processing Systems, pages $33-40,2004$.

[5] Dragomir Anguelov, Praveen Srinivasan, Daphne Koller, Sebastian Thrun, Jim Rodgers, and James Davis. Scape: shape completion and animation of people. ACM Transactions on Graphics, 24(3):408-416, 2005. Proceedings of SIGGRAPH.

[6] Tetsuo Asano, Binay Bhattacharya, Mark Keil, and Frances Yao. Clustering algorithms based on minimum and maximum spanning trees. In Symposium on Computational Geometry, pages 252-257, 1988.

[7] Tetsuo Asano, Prosenjit Bose, Paz Carmi, Anil Maheshwari, Chang Shu, Michiel Smid, and Stefanie Wuhrer. Linear-space algorithm for distance preserving graph embedding with applications. In Canadian Conference on Computational Geometry, pages 185-188, 2007. 
[8] Tetsuo Asano, Prosenjit Bose, Paz Carmi, Anil Maheshwari, Chang Shu, Michiel Smid, and Stefanie Wuhrer. A linear-space algorithm for distance preserving graph embedding. Computational Geometry - Theory and Applications, 42(4):289-304, 2009.

[9] Zouhour Ben Azouz, Prosenjit Bose, Chang Shu, and Stefanie Wuhrer. Approximations of geodesic distances for incomplete triangular manifolds. In Canadian Conference on Computational Geometry, pages 177-180, 2007.

[10] Ilya Baran and Jovan Popović. Automatic rigging and animation of 3d characters. ACM Transactions on Graphics, 26(3):\#72, 1-8, 2007. Proceedings of SIGGRAPH.

[11] Holger Bast. Dimension reduction: A powerful principle for automatically finding concepts in unstructured data. In International Workshop on Self-Properties in Complex Information Systems, pages 113-116, 2004.

[12] Luca Bertelli, Baris Sumengen, and B. S. Manjunath. Redundancy in all pairs fast marching method. In IEEE International Conference on Image Processing, pages 3033-3036, 2006.

[13] Paul Besl and Neil McKay. A method for registration of 3-d shapes. IEEE Transactions on Pattern Analysis and Machine Intelligence, 14(2):239-256, 1992.

[14] Ingwer Borg and Patrick Groenen. Modern Multidimensional Scaling Theory and Applications. Springer, 1997.

[15] Prosenjit Bose, Joseph O'Rourke, Chang Shu, and Stefanie Wuhrer. Isometric morphing of triangular meshes. In Canadian Conference on Computational Geometry, pages $55-58,2008$.

[16] Alexander M. Bronstein, Michael M. Bronstein, and Ron Kimmel. Expression-invariant 3d face recognition. In Audio- and Video-based Biometric Person Authentication, Lecture Notes in Computer Science No. 2688, pages 62-69, 2003.

[17] Alexander M. Bronstein, Michael M. Bronstein, and Ron Kimmel. Three-dimensional face recognition. International Journal of Computer Vision, 64(1):5-30, 2005.

[18] Alexander M. Bronstein, Michael M. Bronstein, and Ron Kimmel. Generalized multidimensional scaling: a framework for isometry-invariant partial surface matching. Proceedings of the National Academy of Sciences, 103(5):1168-1172, 2006. 
[19] Alexander M. Bronstein, Michael M. Bronstein, and Ron Kimmel. Robust expressioninvariant face recognition from partially missing data. In European Conference on Computer Vision, pages 396-408, 2006.

[20] Alexander M. Bronstein, Michael M. Bronstein, and Ron Kimmel. Calculus of non-rigid surfaces for geometry and texture manipulation. IEEE Transactions of Visualization and Computer Graphics, 13(5):902-913, 2007.

[21] Alexander M. Bronstein, Michael M. Bronstein, and Ron Kimmel. Rock, paper, and scissors: extrinsic vs. intrinsic similarity of non-rigid shapes. In International Conference on Computer Vision, pages 1-6, 2007.

[22] Alexander M. Bronstein, Michael M. Bronstein, Ron Kimmel, and Irad Yavneh. Multigrid multidimensional scaling. Numerical Linear Algebra with Applications, Special issue on multigrid methods, 13(2-3):149-171, 2006.

[23] Andreas Buja, Deborah Swayne, Michael Littman, Nate Dean, Heike Hofmann, and Lisha Chen. Interactive data visualization with multidimensional scaling. Journal of Computational and Graphical Statistics, 17(2):444-472, 2008.

[24] Mihai Bădoiu, Erik D. Demaine, Mohammad Taghi Hajiaghayi, and Piotr Indyk. Low-dimensional embedding with extra information. In Symposium on Computational Geometry, pages 320-329, 2004.

[25] Mihai Bădoiu, Kedar Dhamdhere, Anupam Gupta, Yuri Rabinovich, Harald Räcke, R. Ravi, and Anastasios Sidiropoulos. Approximation algorithms for low-distortion embeddings into low-dimensional spaces. In ACM-SIAM Symposium on Discrete Algorithms, pages 119-128, 2005.

[26] Ho-Lun Cheng, Herbert Edelsbrunner, and Ping Fu. Shape space from deformation. In Pacific Conference on Computer Graphics and Applications, pages 104-113, 1998.

[27] Kenneth L. Clarkson. A randomized algorithm for closest-point queries. SIAM Journal on Computing, 17(4):830-847, 1988.

[28] Trevor Cox and Michael Cox. Multidimensional Scaling, Second Edition. Chapman \& Hall CRC, 2001. 
[29] James Davis, Stephen Marschner, Matt Garr, and Marc Levoy. Filling holes in complex surfaces using volumetric diffusion. In International Symposium on 3D Data Processing, Visualization, Transmission, pages 428-438, 2002.

[30] Mark de Berg, Otfried Cheong, Marc van Kreveld, and Mark Overmars. Computational Geometry: Algorithms and Applications, Third Edition. Springer, 2008.

[31] Ian Dryden and Kanti Mardia. Statistical Shape Analysis. Wiley, 2002.

[32] Richard Duda, Peter Hart, and David Stork. Pattern Classification, Second Edition. John Wiley \& Sons, Inc., 2001.

[33] Ilya Eckstein, Jean-Philippe Pons, Yiying Tong, C. C. Jay Kuo, and Mathieu Desbrun. Generalized surface flows for mesh processing. In Symposium on Geometry Processing, pages $183-192,2007$.

[34] Asi Elad and Ron Kimmel. On bending invariant signatures for surfaces. IEEE Transactions on Pattern Analysis and Machine Intelligence, 25(10):1285-1295, 2003.

[35] Yuval Eldar, Michael Lindenbaum, Moshe Porat, and Yehoshua Zeevi. The farthest point strategy for progressive image sampling. IEEE Transactions on Image Processing, 6(9):1305-1315, 1997.

[36] Michael S. Floater and Kai Hormann. Surface parameterization: a tutorial and survey. In Neil A. Dodgson, Michael S. Floater, and Malcolm A. Sabin, editors, Advances in multiresolution for geometric modelling, pages 157-186. Springer Verlag, 2005.

[37] Gene Golub and Charles van Loan. Matrix Computations, Third Edition. The John Hopkins University Press, 1996.

[38] Teofilo Gonzalez. Clustering to minimize the maximum inter cluster distance. Theoretical Computer Science, 38:293-306, 1985.

[39] John C. Gower. Some distance properties of latent root and vector methods used in multivariate analysis. Biometrika, 53:325-338, 1966.

[40] John C. Gower. Adding a point to vector diagrams in multivariate analysis. Biometrika, $55(3): 582-585,1968$. 
[41] Patrick Groenen and Philip H. Franses. Visualizing time-varying correlations across stock markets. Journal of Empirical Finance, 7:155-172, 2000.

[42] Masaki Hilaga, Yoshihisa Shinagawa, Taku Kohmura, and Tosiyasu L. Kunii. Topology matching for fully automatic similarity estimation of 3d shapes. In Special Interest Group on GRAPHics and Interactive Techniques, pages 203-212, 2001.

[43] Dorit S. Hochbaum and David B. Shmoys. A unified approach to approximation algorithms for bottleneck problems. Journal of the ACM, 33(3):533-550, 1986.

[44] Hugues Hoppe. Progressive meshes. In Special Interest Group on GRAPHics and Interactive Techniques, pages 99-108, 1996.

[45] Qixing Huang, Bart Adams, Martin Wicke, and Leonidas J. Guibas. Non-rigid registration under isometric deformations. Computer Graphics Forum (Special Issue of Symposium on Geometry Processing 2008), 27(5):1449-1457, 2008.

[46] Hayley N. Iben, James F. O'Brien, and Erik D. Demaine. Refolding planar polygons. In Symposium on Computational Geometry, pages 71-79, 2006.

[47] Piotr Indyk. Dimensionality reduction techniques for proximity problems. In Symposium on Discrete Algorithms, pages 371-378, 2000.

[48] Piotr Indyk. Nearest Neighbors in High-Dimensional Spaces. In: Jacob E. Goodman and Joseph O'Rourke (Editors). Handbook of Discrete and Computational Geometry (2nd edition). Chapter 39, pages 877892. CRC Press, 2004.

[49] Varun Jain and Hao Zhang. A spectral approach to shape-based retrieval of articulated 3d models. Computer-Aided Design, 39(5):398-407, 2007.

[50] Varun Jain, Hao Zhang, and Oliver van Kaick. Non-rigid spectral correspondence of triangle meshes. International Journal on Shape Modeling, 13(1):101-124, 2007.

[51] Doug L. James and Christopher D. Twigg. Skinning mesh animations. ACM Transactions on Graphics, 24(3):399-407, 2005. Proceedings of SIGGRAPH.

[52] Sagi Katz, George Leifman, and Ayellet Tal. Mesh segmentation using feature point and core extraction. The Visual Computer, 21(8-10):865-875, 2005. 
[53] Sagi Katz and Ayellet Tal. Hierarchical mesh decomposition using fuzzy clustering and cuts. ACM Transactions on Graphics, 22(3):954-96, 2003.

[54] David Kendall. Shape manifolds, procrustean metrics and complex projective spaces. Bulletin of the London Mathematical Society, 16:81-121, 1984.

[55] Martin Kilian, Niloy J. Mitra, and Helmut Pottmann. Geometric modeling in shape space. ACM Transactions on Graphics, 26(3):\#64, 1-8, 2007. Proceedings of SIGGRAPH.

[56] Ron Kimmel and James Sethian. Computing geodesic paths on manifolds. Proceedings of the National Academy of Sciences, 95:8431-8435, 1998.

[57] Danil Kirsanov. Minimal Discrete Curves And Surfaces. PhD thesis, Harvard University, Applied Mathematics, 2001.

[58] Eric Klassen, Anuj Srivastava, Washington Mio, and Shantanu Joshi. Analysis of planar shapes using geodesic paths on shape spaces. IEEE Transactions on Pattern Analysis and Machine Intelligence, 26:372-383, 2004.

[59] Jon Kleinberg and Eva Tardos. Algorithm Design. Addison-Wesley, 2005.

[60] Vladislav Kraevoy and Alla Sheffer. Mean-value geometry encoding. International Journal of Shape Modeling, 12(1):29-46, 2006.

[61] Vladislav Kraevoy, Alla Sheffer, and Craig Gotsman. Matchmaker: Constructing constrained texture maps. ACM Transactions on Graphics, 22(3):326-333, 2003. Proceedings of SIGGRAPH.

[62] Vladislav Kraevoy, Alla Sheffer, and Craig Gotsman. Mean-value geometry encoding. International Journal of Shape Modeling, 12(1):29-46, 2006.

[63] Joseph Kruskal and Myron Wish. Multidimensional Scaling. Sage, 1978.

[64] Dan Kushnir, Meirav Galun, and Achi Brandt. Fast multiscale clustering and manifold identification. Pattern Recognition, 39(10):1876-1891, 2006.

[65] Francis Lazarus and Anne Verroust. Three-dimensional metamorphosis: a survey. The Visual Computer, 14:373-389, 1998. 
[66] Tong-Yee Lee, Yu-Shuen Wang, and Tai-Guang Chen. Segmenting a deforming mesh into near-rigid components. The Visual Computer, 22(9):729-739, 2006.

[67] Marius Leordeanu and Martial Hebert. A spectral technique for correspondence problems using pairwise constraints. In IEEE International Conference on Computer Vision, pages 1482-1489, 2005.

[68] John Lewis, Matt Cordner, and Nickson Fong. Pose space deformation: A unified approach to shape interpolation and skeleton-driven deformation. In Special Interest Group on GRAPHics and Interactive Techniques, pages 165-172, 2000.

[69] Xinju Li and Igor Guskov. Multi-scale features for approximate alignment of pointbased surfaces. In Symposium on Geometry Processing, pages 217-226, 2005.

[70] Jyh-Ming Lien and Nancy M. Amato. Approximate convex decomposition of polygons. Computational Geometry: Theory and Applications, 35(1):100-123, 2006.

[71] Peter Lindstrom and Greg Turk. Fast and memory efficient polygonal simplification. In IEEE Visualization, pages 279-286, 1998.

[72] Yaron Lipman, Olga Sorkine, David Levin, and Daniel Cohen-Or. Linear rotationinvariant coordinates for meshes. ACM Transactions on Graphics, 24(3):479-487, 2005. Proceedings of SIGGRAPH.

[73] Dong C. Liu and Jorge Nocedal. On the limited memory method for large scale optimization. Mathematical Programming, 45:503-528, 1989.

[74] Rong Liu and Hao Zhang. Mesh segmentation via spectral embedding and contour analysis. Computer Graphics Forum (Special Issue of Eurographics 2007), 26:385-394, 2007.

[75] Antoine Maintz and Max Viergever. A survey of medical image registration. Medical Image Analysis, 2(1):1-36, 1998.

[76] Stefan Meiser. Point location in arrangements of hyperplanes. Information and Computation, 106(2):286-303, 1993.

[77] Joseph Mitchell. Geometric shortest paths and network optimizations. In: Jörg-Rüdiger Sack and Jorge Urrutia (Editors). The handbook of computational geometry. Chapter 15, pages 633-701. Elsevier Science, 2000. 
[78] Joseph Mitchell, David Mount, and Christoph Papadimitriou. The discrete geodesic problem. SIAM Journal on Computing, 16:647-668, 1987.

[79] Carsten Moenning and Neil Dodgson. Fast marching farthest point sampling. Technical Report 562, University of Cambridge, Computer Laboratory, 2003.

[80] James Munkres. Algorithms for the assignment and transportation problems. Journal of the Society of Industrial and Applied Mathematics, 5(1):32-38, 1957.

[81] Massimiliano Pavan and Marcello Pelillo. Dominant sets and hierarchical clustering. In IEEE International Conference on Computer Vision, pages 362-369, 2003.

[82] Massimiliano Pavan and Marcello Pelillo. Dominant sets and pairwise clustering. IEEE Transactions on Pattern Analysis and Machine Intelligence, 29(1):167-172, 2007.

[83] Gabriel Peyre and Laurent Cohen. Geodesic computations for fast and accurate surface remeshing and parameterization. Progress in nonlinear differential equations and their applications, 63:157-171, 2005.

[84] Willian Press, Brian Flannery, Saul Teukolsky, and William Vetterling. Numerical Recipes in C: the Art of Scientific Computing. Cambridge University Press, 1993.

[85] Kathleen Robinette, Hans Daanen, and Eric Paquet. The caesar project: A 3-d surface anthropometry survey. In 3-D Digital Imaging and Modeling, pages 180-186, 1999.

[86] Guy Rosman, Alexander M. Bronstein, Michael M. Bronstein, and Ron Kimmel. Topologically constrained isometric embedding. Human Motion Understanding, Modelling, Capture, and Animation, 36:243-262, 2008.

[87] Sam Roweis and Lawrence Saul. Nonlinear dimensionality reduction by locally linear embedding. Science, 190(5500):2323-2326, 2000.

[88] Mirko Sattler, Ralf Sarlette, and Reinhard Klein. Simple and efficient compression of animation sequences. In Symposium on Computer Animation, pages 209-217, 2005.

[89] John Schreiner, Arul Asirvatham, Emil Praun, and Hugues Hoppe. Inter-surface mapping. ACM Transactions on Graphics, 23(3):870-877, 2004. Proceedings of SIGGRAPH. 
[90] Thomas W. Sederberg, Peisheng Gao, Guojin Wang, and Hong Mu. 2-d shape blending: an intrinsic solution to the vertex path problem. In Special Interest Group on GRAPHics and Interactive Techniques, pages 15-18, 1993.

[91] James Sethian. A fast marching level set method for monotonically advancing fronts. Proceedings of the National Academy of Sciences, 93(4):1591-1595, 1996.

[92] James Sethian. Level Set Methods and Fast Marching Methods: Evolving Interfaces in Computational Geometry, Fluid Mechanics, Computer Vision, and Materials Science, Second Edition. Cambridge University Press, 1999.

[93] Ariel Shamir. A survey on mesh segmentation techniques. Computer graphics forum, $27(6): 1539-1556,2008$.

[94] Alla Sheffer, Emil Praun, and Kenneth Rose. Mesh parameterization methods and their applications. Foundations and Trends in Computer Graphics and Vision, 2(2):105-171, 2006.

[95] Philip Shilane, Patrick Min, Michael Kazhdan, and Thomas Funkhouser. The princeton shape benchmark. In Proceedings of Shape Modeling International, 2004.

[96] Ken Shoemake. Animating rotation with quaternion curves. In Special Interest Group on GRAPHics and Interactive Techniques, pages 245-254, 1985.

[97] Jeremy G. Siek, Lie-Quan Lee, and Andrew Lumsdaine. The boost graph library: user guide and reference manual. Addison-Wesley Longman Publishing Co., Inc., 2002.

[98] Olga Sorkine and Marc Alexa. As-rigid-as-possible surface modeling. In Symposium on Geometry processing, pages 109-116, 2007.

[99] Robert W. Sumner and Jovan Popović. Deformation transfer for triangle meshes. ACM Transactions on Graphics, 23(3):399-405, 2004. Proceedings of SIGGRAPH.

[100] Yue Man Sun, Wengping Wang, and Francis Chin. Interpolating polyhedral models using intrinsic shape parameters. The Journal of Visualization and Computer Animation, 8(2):81-96, 1997.

[101] Vitaly Surazhsky and Craig Gotsman. Intrinsic morphing of compatible triangulations. International Journal of Shape Modeling, 9:191-201, 2003. 
[102] Vitaly Surazhsky, Tatiana Surazhsky, Danil Kirsanov, Steven J. Gortler, and Hugues Hoppe. Fast exact and approximate geodesics on meshes. ACM Transactions on Graphics, 24(3):553-560, 2005. Proceedings of SIGGRAPH.

[103] Joshua B. Tenenbaum, Vin de Silva, and John C. Langford. A global geometric framework for nonlinear dimensionality reduction. Science, 190(5500):2319-2323, 2000.

[104] Julien Tierny, Jean-Philippe Vandeborre, and Mohamed Daoudi. Invariant high level reeb graphs of 3D polygonal meshes. In IEEE International Symposium on 3D Data Processing Visualization Transmission, pages 105-112, 2006.

[105] Julien Tierny, Jean-Philippe Vandeborre, and Mohamed Daoudi. Enhancing 3d mesh topological skeletons with discrete contour constrictions. Visual Computer, 24(3):155-172, 2008.

[106] Sébastien Valette and Jean-Marc Chassery. Approximated centroidal voronoi diagrams for uniform polygonal mesh coarsening. Computer Graphics Forum (Special issue of Eurographics 2004), 23(3):381-390, 2004.

[107] George Wolberg. Image morphing: a survey. The Visual Computer, 14:360-372, 1998.

[108] Stefanie Wuhrer, Prosenjit Bose, Chang Shu, Joseph ORourke, and Alan Brunton. Morphing of triangular meshes in shape space. International Journal of Computational Geometry and Applications, submitted, see arXiv:0805.0162v2, 2008.

[109] Stefanie Wuhrer and Alan Brunton. Segmenting triangular meshes into near-rigid components. The Visual Computer, submitted, see NRC 50724/ERB-1155, 2008.

[110] Stefanie Wuhrer, Chang Shu, Zouhour Ben Azouz, and Prosenjit Bose. Posture invariant correspondence of incomplete triangular manifolds. International Journal of Shape Modeling, 13(2):139-157, 2007.

[111] Stefanie Wuhrer, Chang Shu, and Prosenjit Bose. Posture invariant correspondence of triangular meshes in shape space. IEEE International Conference on Computer Vision 2009, submitted.

[112] Pengcheng Xi, Won-Sook Lee, and Chang Shu. Analysis of segmented human body scans. In Graphics Interface, pages 19-26, 2007. 
[113] Hussein Yahia, Etienne Huot, Isabelle Herlin, and Isaac Cohen. Geodesic distance evolution of surfaces: A new method for matching surfaces. IEEE Conference on Computer Vision and Pattern Recognition, 1:663-668, 2000.

[114] Liron Yatziv, Alberto Bartesaghi, and Guillermo Sapiro. O(n) implementation of the fast marching algorithm. Journal of Computational Physics, 212(2):393-399, 2006.

[115] Laurent Younes. Optimal matching between shapes via elastic deformations. Journal of Image and Vision Computing, 17(5,6):381-389, 1999.

[116] Hao Zhang, Alla Sheffer, Daniel Cohen-Or, Qingnan Zhou, Oliver van Kaick, and Andrea Tagliasacchi. Deformation-driven shape correspondence. Computer Graphics Forum (Special Issue of Symposium on Geometry Processing 2008), 27(5):1431-1439, 2008 .

[117] Juan Zhangand, Kaleem Siddiqi, Diego Macrini, Ali Shokoufandeh, and Sven Dickinson. Retrieving articulated 3-d models using medial surfaces and their graph spectra. In International Workshop On Energy Minimization Methods in Computer Vision and Pattern Recognition, pages 285-300, 2005.

[118] Kun Zhou, John Snyder, Baining Guo, and Heung-Yeung Shum. Iso-charts: Stretchdriven mesh parameterization using spectral analysis. In Eurographics Symposium on Geometry Processing, pages 45-54, 2004.

[119] Gil Zigelman, Ron Kimmel, and Nahum Kiryati. Texture mapping using surface flattening via multi-dimensional scaling. IEEE Transactions on Visualization and Computer Graphics, 8(2):198-207, 2002.

[120] Barbara Zitova and Jan Flusser. Image registration methods: a survey. Image and Vision Computing, 21(11):977-1000, 2003. 